\title{
WestVirginiaUniversity
}

THE RESEARCH REPOSITORY @ WVU

Graduate Theses, Dissertations, and Problem Reports

2020

\section{Searches for Fast Radio Bursts}

\author{
Golnoosh Golpayegani \\ gogolpayegani@mix.wvu.edu
}

Follow this and additional works at: https://researchrepository.wvu.edu/etd

\section{Recommended Citation}

Golpayegani, Golnoosh, "Searches for Fast Radio Bursts" (2020). Graduate Theses, Dissertations, and Problem Reports. 7545.

https://researchrepository.wvu.edu/etd/7545

This Dissertation is protected by copyright and/or related rights. It has been brought to you by the The Research Repository @ WVU with permission from the rights-holder(s). You are free to use this Dissertation in any way that is permitted by the copyright and related rights legislation that applies to your use. For other uses you must obtain permission from the rights-holder(s) directly, unless additional rights are indicated by a Creative Commons license in the record and/ or on the work itself. This Dissertation has been accepted for inclusion in WVU Graduate Theses, Dissertations, and Problem Reports collection by an authorized administrator of The Research Repository @ WVU.

For more information, please contact researchrepository@mail.wvu.edu. 


\title{
Searches for Fast Radio Bursts \\ Golnoosh Golpayegani
}

\author{
Dissertation Submitted to \\ The Eberly College of Arts and Sciences \\ at West Virginia University \\ in partial fulfillment of the requirements \\ for the degree of \\ Doctor of Philosophy \\ in \\ Physics \\ Duncan Lorimer, Ph.D., Chair \\ Maura McLaughlin, Ph.D. \\ Loren Anderson, Ph.D. \\ Majid Jaridi, Ph.D. \\ Morgantown, West Virginia, USA \\ 2020
}

Keywords: pulsars, radio transients, FRBs

Copyright 2020 Golnoosh Golpayegani 
Abstract

\title{
Searches for Fast Radio Bursts
}

\author{
Golnoosh Golpayegani
}

Following the discovery of fast radio bursts (FRBs) in 2007, astronomers have entered a new era in astronomy in which understanding the nature of these type of radio transients is one of the most important modern astronomy questions. In this thesis I detail our current state of knowledge in this rapidly evolving field and describe real-time search systems designed to find FRBs using the 20-meter radio telescope at the Green Bank Observatory and the Arecibo L-band Feed Array (ALFA) receiver of the Arecibo 300-meter telescope in Puerto Rico. These experiments are called GBTrans and ALFABURST, respectively.

I give details of the observing systems and report on the non-detection of FRBs for both surveys. GBTrans is sensitive enough to detect approximately half of all currently known FRBs while ALFABURST is sensitive enough to detect almost all of the current FRB population. I estimate that GBTrans survey probed redshifts out to about 0.3 corresponding to an effective survey volume of around 124,000 $\mathrm{Mpc}^{3}$. Assuming a constant density for sources per unit co-moving volume and considering the possibility of detecting bright FRBs in the sidelobes of the ALFA beams, I estimate ALFABURST probed redshifts out to about 3.5. Based on this, the expected event rate would be at most two FRBs per year at the $99 \%$ confidence level. Modeling the FRB rate as a function of fluence, $F$, as a power law with $F^{-\alpha}$, I constrain the index $\alpha<2.5$ at the $90 \%$ confidence level based on the GBTrans results.

A number of pulses from previously known pulsars were detected in both the GBTrans and ALFABURST surveys which provided excellent verification on the survey sensitivity used to compute the effective volumes quoted above. One Galactic transient, J1845+00, was found in the ALFABURST survey. This is most likely a member of the rotating radio transient (RRAT) population. It has so far not been seen in follow-up observations. Eight further single-pulse candidates from ALFABURST are also reported. At the time of writing, due to incomplete metadata records, the positions of these sources are not well enough known to allow further follow-up. Future observations with ALFABURST are anticipated in the coming year. Finally, I also describe preliminary observations from an Arecibo survey of gamma-ray burst sources. 


\section{Dedication}

To my parents, Minoo Tayebi and Hadi Golpayegani: because I owe it all to you. 


\section{Acknowledgements}

Undertaking this PhD has been truly a life-changing challenge for me and this dissertation would not have been completed without the support and guidance that I received from many people. I would like to pay my deepest gratitude to my dear advisor, Dr. Duncan Lorimer. His never-ending support and encouragement started from day one, when he kindly responded to my very first inquiry email from Iran and since then, he has always been there for me whenever I needed help and patiently kept me motivated. I definitely could never find a better advisor both in immense knowledge and being a wonderful human being. Thanks to him, besides from data analysis and theoretical astrophysics, I will benefit from all the writing and presentation skills for the rest of my life. He generously provided me with every opportunity to travel and kindly sent me to UC Berkeley to finish my research in a different atmosphere which changed my life. Dunc, I could not have imagined having a better advisor and mentor for my $\mathrm{PhD}$, I honestly cannot find proper words to describe my gratitude.

I would like to thank my dissertation committee members for their useful feedback and comments on this dissertation: Special thanks to Dr. Maura McLaughlin with whom I worked on a couple of projects. She has always been a role model as a strong woman in science for me and I have learned a lot from her. She raised many precious points in our discussion and I hope that I have managed to address several of them here. I would like to thank Dr. Loren Anderson for his insightful comments and for all the positive energy and interesting topics I learned from his class, and I would also like to recognize Dr. Majid Jaridi for all his encouragement and valuable input which incentivized me to widen my research from various perspectives.

I would like to acknowledge the astro faculty in the department, especially Dr. Kathryn Williamson for being a great outreach mentor and the department chair, Dr. D.J. Pisano for being supportive and positive at all times. I would also like to thank all the department office staff, Sherry Puskar, Beena Bhanushali, and specially Viola Bryant for their efficient handling of all the necessary paperwork. A big thank you to Nate Garver-Daniels for fixing all the network problems, and to Margaret Mattson for assisting me with all the travelling paperwork.

I would also like to thank my collaborators, Dr. Steven Ellingson, Dr. Mayuresh Surnis, Dr. Griffin Foster, Dr. Nipuni Palliyaguru, Dr. Aris Karastergiou, Dr. Kaustubh Rajwade, Dr. Jayanth Chennamangalam, Dr. Frank Ghigo, Olivia Young, Michael Mingyar, and the late Dr. Richard Prestage. Big thanks to Arun Venkataraman at the Arecibo Observatory for his assistance with remote observations. I would like to recognize the invaluable help that Devansh Agarwal provided me during the last two years of my $\mathrm{PhD}$ on our mutual projects.

I am very grateful to my fellow graduate students at the M59 office: Dr. Akshaya Rane, Dr. Will Armentrout, Dr. Amy Sardone, Dr. Megan Jones, Dr. Matteo Luisi, Nihan Pol, Josh Mascoop, Belinda Cheeseboro, Amber Lenon, Joseph Kania, Kshitij Aggarwal, Brent Shapiro-Albert, and Caitlin Witt for their great company, stimulating discussions on research projects, our productive lunch discussions, and supporting me in tough times. You all made coming to the office a fun experience 
over the past six years. I am thankful to Pedram Tavazohi and Tommy Steinberger for the sleepless nights we were studying together before the qualifying exams. I am also grateful to the senior graduate students and postdocs in the department, especially Dr. Natalia Lewandowska for being a cool travel buddy, Dr. Peter Gentile for helping me with Python, Dr. Michael Lam, Dr. Joseph Swiggum, Dr. Lucas Hunt, Dr. Nick Pingel, Dr. Paul Brook, and Fernando Cardoso.

I am forever indebted to Dr. Dan Werthimer for giving me the fantastic opportunity to join his team at UC Berkeley during my last year of the $\mathrm{PhD}$ and for giving me access to RAL lab and research facilities. I am also grateful to him and his lovely family, Dr. Mary Kate Morris and Willy Werthimer, who opened their home and heart to me and for helping me settle in Berkeley. Dan, without your invaluable support, it would not be possible to finish this work and I whole-heartedly appreciate you checking on me everyday, giving me different tours in Berkeley and giving me great advice for a whole year. I am also very grateful to all those at the Berkeley SETI Research Center, especially Dr. Andrew Seimion, Dr. Vishal Gajjar, Dr. Jack Hickish, Dr. Casey Law, Dr. Howard Isaacson, Dr. David Macmahon, Matt Lebofsky, Dr. Steve Croft, Jeff Cobb, and Dr. Emilio Enriquez for their hospitality and for inviting me to their group meetings and happy hours, where I learned a lot and had so much fun. I am also thankful to Mark Hayden at Campbell Hall for helping me through all the paperwork during my visit.

Getting through this dissertation required more than academic support, and I have many, many people to thank; for listening to me and, at times, having to tolerate me over the past six years. I cannot begin to express my gratitude and appreciation for their friendship. A big thanks to doctor-to-be Faezeh Sedighi who was there for me through the thick and thin like a sister, Dr. Pariya Pourmohammad for her positive energy, everyone in the WVU Latin Dance Club, Dr. Shahab Mohaghegh, Tony Pompa, Mahsa Kiani, Connie Ahrens, and Caitlin Ahrens. To my dearest friends in Tehran, London, and Toronto: Bahar Noorizadeh, Afsoon Jafari, Yassaman Momeni, and Negar Kermanshahi: thanks for being there for me whenever I needed to talk in spite of being on different continents. I would also like to thank all of my friends who supported me in writing while I was in Berkeley: Amin Ebrahimi, Yasaman Sedaghat, Khandan Bigdeli, Carole Burns, Robert Shepard, and my friends at Maestro Beyzaie's workshop: Ali Kamran, Sara Safdari, Homa Harati, Ghazal Hemmat, Dr. Nasim Gorgani, Behzad Golemohammadi, and Leila Ghazavi. I would also like to acknowledge my high school astronomy teacher, Dr. Shahin Jafarzadeh, and my undergraduate supervisor, Dr. Amirsina Shaibani for being so awesome in motivating me to pursue a degree in astronomy.

Most importantly, none of this could have happened without the moral support of my dear family, which was instrumental in the completion of this dissertation. I owe my biggest thanks to my parents: Minoo Tayebi and Hadi Golpayegani, for everything they have done to bolster my interest in astronomy since childhood and for all the love and support throughout this long journey in grad school. They went on a ride along with me with many ups and downs, kept helping me push to overcome difficulties and never gave up even though their only child was thousands of miles apart for six consecutive years until the finish line. Words cannot express 
how grateful I am to my mom and dad for all of the sacrifices they made on my behalf. I would also like to thank my family in the US. A very special thanks to my angel aunt, Dr. Nahid Tayebi, without whom, I would not have had the courage to embark on this journey in the first place. Thank you for all your unconditional love and support, Khale. I would also like to say a heartfelt thank you to my uncle Dr. Kazem Kazemour for his invaluable advice and for always being so supportive of my studies. Thanks to my cousins in DC, Ozhan and Denna Kazempour, for always being there for me like real siblings. Thanks to my uncle Javad Tayebi and my aunt Dr. Kandi Tayebi, and my cousins Bezhan and Shayon for sending me their love and support from Houston. I would also like to thank my cousin Dr. Amin Tayebi and his lovely wife Alicia for helping me get settled on the West Coast and never letting me feel alone. I would also want to thank my sweet grandmother Ashraf Mirmomeni, who offered her nice words through video calls, despite my own limited devotion to correspondence. Thanks to my beloved uncle Alireza Tayebi and Saedeh Soltan, and my darling aunt Ashraf Golpayegani for the constant love they sent me from Tehran and Berlin. And finally thanks to Memol, who has been patiently and loyally by my side throughout this $\mathrm{PhD}$, living every single minute of it. This dissertation stands as a testament to the unconditional love and encouragement I received from every single one of you. 


\section{Table of Contents}

List of Tables $\quad$ ix

List of Figures $\quad$ x

1 Introduction 1

1.1 Overview of the FRB phenomenon . . . . . . . . . . . . . 3

1.1.1 Sky Distribution . . . . . . . . . . . . . 3

1.1 .2 Dispersion . . . . . . . . . . . . . . . 4

1.1.3 Scattering . . . . . . . . . . . . . . . . 7

1.1.4 Scintillation . . . . . . . . . . . . . 10

1.1.5 Polarization .................... 11

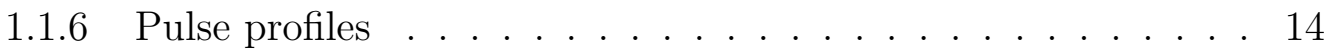

1.1 .7 Redshifts . . . . . . . . . . . . . . . . 16

1.1.8 Energetics . . . . . . . . . . . . . . . 20

1.1.9 Source counts and event rates . . . . . . . . . . . . 23

1.2 Historical development of the field . . . . . . . . . . . . . . . 25

1.2.1 The Lorimer Burst (FRB 010724) . . . . . . . . . . . . . . . . 25

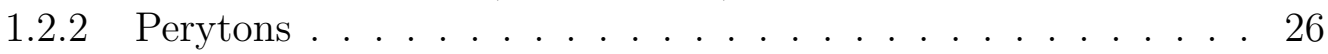

1.2.3 The Keane Burst (FRB 010621) . . . . . . . . . . . . . . . . . 27

1.2.4 Thornton Bursts (FRBs 110220, 110627, 110703 and 120127) . 29

1.2.5 The first repeating source, FRB 121102 . . . . . . . . . . 30

1.2.6 A second repeating source (FRB 180814.J0422+73) . . . . . . 31

1.2.7 Localized non-repeating FRBs . . . . . . . . . . . . . . . . . 34

1.2.8 More Repeating FRBs . . . . . . . . . . . . . . . . 34

1.3 Thesis Outline . . . . . . . . . . . . . . . . . . . 35

2 Models for FRBs 38

2.1 Isolated Neutron Star Models . . . . . . . . . . . . . . . . . . . 39

2.1.1 Giant Pulses . . . . . . . . . . . . . . . . . . . . . 39

2.1.2 Starquakes........................ 39

2.2 Magnetar Giant Flares . . . . . . . . . . . . . . . . . . . . . . . . 40

2.3 Merging and Colliding Neutron Star Models . . . . . . . . . . . . . 41

2.4 Collapsing Neutron Star Models . . . . . . . . . . . . . . . . 43

2.5 Compact Object Interaction Models . . . . . . . . . . . . . . . . . 45

2.5.1 Compact Object Binary Systems . . . . . . . . . . . . 45

2.5.2 Pulsar-orbiting Body Interactions . . . . . . . . . . . . . 46

2.5.3 Cosmic Combs . . . . . . . . . . . . . . . . . . 48

2.5.4 Dark Matter Models . . . . . . . . . . . . . . . . . . . 49

2.6 Black Hole Progenitors . . . . . . . . . . . . . . . . . . . . . . . . 49

2.6.1 Dark Matter-induced Collapses . . . . . . . . . . . . . . . 49

2.6.2 Neutron Star-Black Hole Mergers . . . . . . . . . . . . . . . . 50

2.6.3 Black Hole-Black Hole Mergers . . . . . . . . . . . . . . . . . 50

2.6.4 Jet-Caviton Models . . . . . . . . . . . . . . . . . . 51 
2.7 Exotic Models . . . . . . . . . . . . . . . . . . . . . . . 52

2.7.1 Extraterrestrial Intelligence . . . . . . . . . . . . . . 52

2.7.2 White Holes . . . . . . . . . . . . . . . . . . . . . . 53

2.7 .3 Cosmic Strings . . . . . . . . . . . . . . . . . . 53

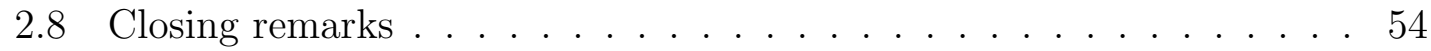

3 Surveys for FRBs 55

3.1 Single-Dish Surveys . . . . . . . . . . . . . . . . . 55

3.2 Interferometric Surveys . . . . . . . . . . . . . . . . . . . 57

3.3 Single-Dish Search Techniques for FRBs . . . . . . . . . . . . 59

3.3 .1 Interference Excision . . . . . . . . . . . . . . . . . . . . 59

3.3.2 De-dispersion . . . . . . . . . . . . . . . . . . . . . . . . . . 61

3.3.3 Single-pulse search . . . . . . . . . . . . . . . . . . . . 62

3.3 .4 Candidate selection . . . . . . . . . . . . . . . . . 64

3.3 .5 Sensitivity . . . . . . . . . . . . . 68

4 A search for FRBs with the Green Bank 20-m Telescope 70

4.1 Introduction . . . . . . . . . . . . . . . . . 70

4.2 GBTrans Description . . . . . . . . . . . . . . . 71

4.3 Observations and Data Processing . . . . . . . . . . . . . . . . 73

4.4 Discussion . . . . . . . . . . . . . . . . . . . . 78

4.4 .1 Expected FRB Rate . . . . . . . . . . . . . . . . . 78

4.4 .2 Survey Volume . . . . . . . . . . . . . . . . . . 84

5 A search for FRBs with the Arecibo telescope 85

5.1 Introduction . . . . . . . . . . . . . . . 85

5.2 Observations . . . . . . . . . . . . . . . . 86

5.2 .1 ALFA Description . . . . . . . . . . . . . 86

5.2 .2 ALFABURST description . . . . . . . . . . . . . . 87

5.2 .3 Radio Frequency Interference Excision . . . . . . . . . . 92

5.2 .4 Commissioning of ALFABURST . . . . . . . . . . . . . . . 94

5.2 .5 Post-processing Pipeline . . . . . . . . . . . . . . . 95 95

5.3 Results and Discussion . . . . . . . . . . . . . . 96

5.3.1 Previously published results . . . . . . . . . . . . 96

5.3.2 Recent Search Results . . . . . . . . . . . . . . . . . . . 104

5.3 .3 Survey Sensitivity . . . . . . . . . . . . . . . . . 106

5.3 .4 Discussion . . . . . . . . . . . . . . . . . . . 107

6 Conclusions 110

6.1 GBTrans constraints on Galactic FRBs . . . . . . . . . . . . . . . 110

6.2 Source counts revisited . . . . . . . . . . . . . . . . . 111

6.3 Future FRB searches with $20 \mathrm{~m}$ telescope . . . . . . . . . . . . . 112

6.4 ALFABURST . . . . . . . . . . . . . . . . . . . . . 115

6.5 Current FRB searches with Arecibo and the GBT . . . . . . . 116

6.6 Going Forward . . . . . . . . . . . . . . . . . . . 120 


\section{List of Tables}

1.1 Summary of published FRB rates . . . . . . . . . . . . . . . . 24

1.2 Properties the repeating FRBs . . . . . . . . . . . . . . 37

3.1 Summary of FRB surveys . . . . . . . . . . . . . . . . 56

4.1 detected pulsars during the GBTrans survey . . . . . . . . . . . . . 78

4.2 Other constraints on the source count index, $\alpha \ldots \ldots$. . . . . 81

5.1 Detected pulsars in the ALFABURST survey . . . . . . . . . . . . . 97

5.2 Parameters for ALFABURST single-pulse candidates . . . . . . . . 106

6.1 Target GRBs searched at Arecibo . . . . . . . . . . . . . . 118

6.2 Target GRB parameters . . . . . . . . . . . . . . . . . . . . . . . . . 119 


\section{List of Figures}

1.1 FRB sky distribution in Galactic coordinates . . . . . . . . . . . 4

1.2 Pulse dispersion effect shown for PSR B1900+01 . . . . . . . . 6

1.3 Pulse scattering as observed for FRB $110220 \ldots \ldots$. . . . . . . 9

1.4 Dispersion measures and scattering times plotted against Galactic latitude for pulsars and FRBs . . . . . . . . . . . . . . 10

1.5 Scintillation as observed for FRB $170827 \ldots \ldots \ldots \ldots$

1.6 Pulse profiles for twenty-eight FRBs . . . . . . . . . . . . . 15

1.7 The history of cosmic star formation . . . . . . . . . . . . . 19

1.8 Waterfall plot showing the Lorimer burst . . . . . . . . . . . . . 26

1.9 Waterfall plot of FRB $010621 \ldots \ldots \ldots \ldots$

1.10 VLA detection of FRB $121102 \ldots \ldots \ldots \ldots$

1.11 Radio and optical images of the FRB 121102 field . . . . . . . . . . 33

1.12 Waterfall plots showing eight repeating FRBs . . . . . . . . 36

2.1 Schematic showing a unipolar inductor model for FRBs . . . . . . . . 42

2.2 Intermittent Roche-lobe overflow in a neutron star-white dwarf binary system . . . . . . . . . . . . . . . . . 4 4 4

2.3 Schematic showing a cosmic comb model for FRB emission . . . . . . 48

2.4 Schematic view of a Kerr-Newman black hole . . . . . . . . . . . . 51

3.1 Pulse dispersion and de-dispersion . . . . . . . . . . . . . . 63

3.2 Schematic showing a single pulse search procedure . . . . . . . . . 65

3.3 Sample FRB images produced by FETCH . . . . . . . . . . . . 67

4.1 GBTrans block diagram $\ldots \ldots \ldots \ldots . \ldots . \ldots . \ldots 71$

4.2 GBTrans observation dates . . . . . . . . . . . . 74

4.3 Giant pulse from the Crab pulsar . . . . . . . . . . . 76

4.4 Example GBTrans output showing radio-frequency interference . . . . 77

4.5 GBTrans sensitivity curve . . . . . . . . . . . . . . 82

4.6 GBTrans constraints on the FRB source count index . . . . . . . . 83

5.1 ALFA receiver beam pattern . . . . . . . . . . . . 87

5.2 SETIBURST architecture . . . . . . . . . . . . . . . . . . . . 89

5.3 Schematic showing the ARTEMIS Modular Pelican Pipeline . . . . . 91

5.4 ALFABURST bandpass . . . . . . . . . . . . . . . . 94

5.5 ALFABURST detection of a single pulse from PSR B1900+01 . . . 98

5.6 Waterfall plot showing the Galactic transient J1845+00 . . . . . . . 101

5.7 DM-time plot for the Galactic transient J1845+00 . . . . . . . . 102

5.8 ALFABURST sky coverage . . . . . . . . . . . . . . . . . 103

5.9 ALFABURST new single-pulse candidates . . . . . . . . . . 105

5.10 ALFABURST survey sensitivity . . . . . . . . . . . . . . 107

6.1 Source Count Models . . . . . . . . . . . . . . . . . . . . . . . . . . 112

6.2 The new triggered GRB burst alert system design . . . . . . . . . 114 


\section{Chapter 1}

\section{Introduction}

Transient phenomena involving compact objects (white dwarfs, neutron stars, and black holes) are among the most rapidly studied areas of modern astronomy. Like many new scientific fields, transient radio astrophysics was pioneered by serendipity. The first sub-second duration radio pulses were found by a group using a low-frequency array in the UK. From an analysis of these observations, Cambridge University student Jocelyn Bell found the first source of individual pulses with a repetition period of $1.337 \mathrm{~s}$ (Hewish et al., 1968). Since then, almost 3000 further sources, now known as pulsars, have been found and demonstrated to be a population of neutron stars in the disk of the Milky Way, its globular cluster systems and the Magellanic Clouds (for a review, see Lorimer \& Kramer, 2004).

Over the years, pulsar searches and their need for high time and frequency resolution with ever improving radio facilities have opened new windows on the transient universe, which reminds us that the potential for discovery is large. The best example of this so far is the discovery of fast radio bursts (FRBs; Lorimer et al., 2007; Thornton et al., 2013). FRBs are very bright transient radio pulses that occur on short $(\sim m s)$ timescales, but emit about as much energy as the Sun produces in a month. At the time of writing, around a hundred FRBs are in the public domain (for an up-to-date list, see Petroff et al., 2016). The properties of 
the bursts, including their anomalously high dispersion delays, suggest that they likely have a celestial origin. Although this sample is currently not large enough to unambiguously characterize their origin and emission mechanism, it is clear that they form a cosmological population (see, e.g., Caleb et al., 2016; Tendulkar et al., 2017) that is quite distinct from the known pulsars.

Though most FRBs have been detected as one-off events, a few so far have shown repetitions (Spitler et al., 2016; CHIME/FRB Collaboration et al., 2019a; The CHIME/FRB Collaboration et al., 2019; Marcote et al., 2020). Studies of the first of these, FRB 121102, allowed the burst to be localized to a star-forming region in a host dwarf galaxy (Chatterjee et al., 2017), using the Karl G. Jansky Very Large Array (VLA) acting jointly with single-dish observations using the 305m William E. Gordon Telescope at the Arecibo Observatory. A measurement of the redshift of the host galaxy meant that FRB 121102 was the first FRB with a direct distance determination (Tendulkar et al., 2017). Follow-up studies showed a large and variable rotation measure towards this source, suggesting that FRB 121102 is in an extreme and dynamic magneto-ionic environment. A neutron star origin is consistent with both such an environment and the short burst durations (Michilli et al., 2018).

A second repeating FRB, 180814.J0422+73 was recently discovered by CHIME (Canadian Hydrogen Intensity Mapping Experiment CHIME/FRB Collaboration et al., 2019a). The CHIME collaboration reported the detection of six repeat bursts from FRB 180814.J0422+73 among 13 detected FRBs during the telescope's precommissioning phase (CHIME/FRB Collaboration et al., 2019b). Most recently, 
CHIME discovered eight further repeating FRBs, varying from two repeat bursts to ten (The CHIME/FRB Collaboration et al., 2019) including the very recent announcement of a repeating source localized to a nearby spiral galaxy (Marcote et al., 2020). It is not currently clear if the existence of repeating FRBs implies distinct source classes (see, e.g., Caleb et al., 2019).

This thesis aims to further our knowledge of the FRB population(s) through several different observational strategies. To set the scene for this work, in this chapter, I will give a brief description of what is currently known about FRB observables, their detection history, search techniques, the population, FRB surveys and possible progenitor model scenarios that can be found in the literature so far. This field is frequently evolving, and further discussion of these topics can be found in two recent review papers (Petroff et al., 2019; Cordes \& Chatterjee, 2019).

\subsection{Overview of the FRB phenomenon}

\subsubsection{Sky Distribution}

Fig. 1.1 shows the sky distribution in Galactic coordinates of all published FRBs at the time of writing. As can be seen, there is no strong preference for FRB directions along the Galactic plane as there is for radio pulsars. For a cosmological source population, like gamma-ray bursts for example, there should be no preferred direction for FRBs on the sky. Due to the fact that the majority of the first FRBs were discovered by Parkes radio telescope located in Australia, which predominantly

observes the southern hemisphere, some of the earlier analyses suggested that the 


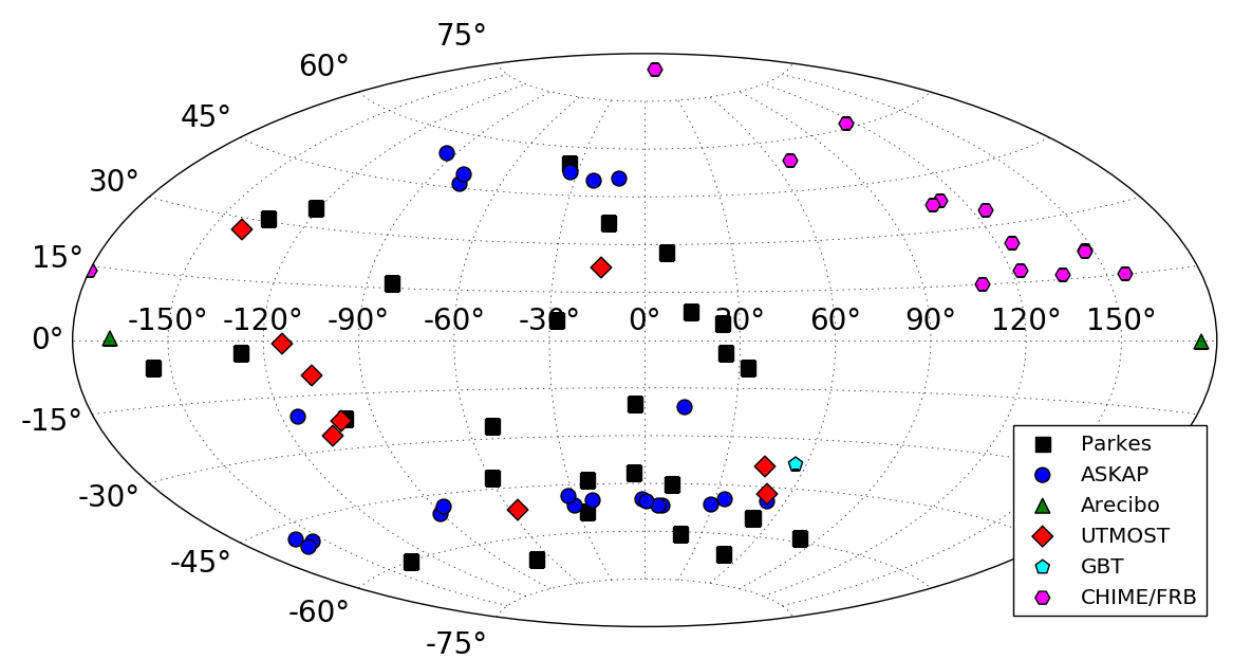

Figure 1.1 An Aitoff projection map of the sky positions of all published FRBs so far. Here the Galactic longitude is shown ranging from -180 to 180 degrees and the Galactic latitude ranges from -90 to +90 degrees. Colors correspond to FRBs from different telescopes. Taken from Petroff et al. (2019).

sky distribution of FRBs is non-uniform (see, e.g., Petroff et al., 2014). Further analysis by Rane et al. (2016), making use of a larger sample, showed that in fact there was no evidence for a latitude dependence on the FRB rate. With the large number of FRBs now being found by CHIME, we anticipate far more detailed studies of the sky distribution in the near future.

\subsubsection{Dispersion}

Radio wave dispersion occurs due to the frequency-dependent group velocity of the radio waves as they propagate through an ionized medium. As a result, the higher frequency pulses arrive earlier than the lower frequency pulses and is seen as a sweep in the frequency versus time plot. Fig. 1.2 shows an example of a pulse with 
this time delay over the observed frequency range. This delay in the arrival time is one of the primary observables of an FRB. The time delay, $\Delta t$, of the pulse arrival over the observing frequency range $\nu_{2}<\nu<\nu_{1}$ is given by

$$
\Delta t=4.15 \times 10^{6} \mathrm{~ms} \times \mathrm{DM} \times\left(\frac{1}{\nu_{1}^{2}}-\frac{1}{\nu_{2}^{2}}\right),
$$

where $\nu_{1}$ and $\nu_{2}$ are in $\mathrm{MHz}$ and the dispersion measure

$$
\mathrm{DM} \equiv \int_{O}^{S} n_{e} d \ell
$$

is the integrated electron density along the propagation line of sight with $n_{e}$ representing the electron number density of the medium, and $\ell$ is the path from the observer $O$ to the source $S$ and is expressed in the hybrid units of $\mathrm{cm}^{-3} \mathrm{pc}$.

From this we infer, for a constant electron density, DM is directly proportional to the distance to the source. Because FRB DMs significantly exceed the maximum predicted contribution from the Galactic interstellar medium (ISM) along each line of sight, we infer that they must be extragalactic. However, it is necessary to determine how much of this dispersion is from progenitor itself, the intergalactic medium (IGM), and the host galaxy to better constrain the FRB's distance and energetics. We can set some constraints from the observed DM as follows. If we define the dispersion measure excess $\left(\mathrm{DM}_{\mathrm{E}}\right)$ to be the DM subtracted from the Milky Way DM contribution from the line of sight, which can be estimated from a model for Galactic electron density like the NE2001 model (Cordes \& Lazio, 2002), then 


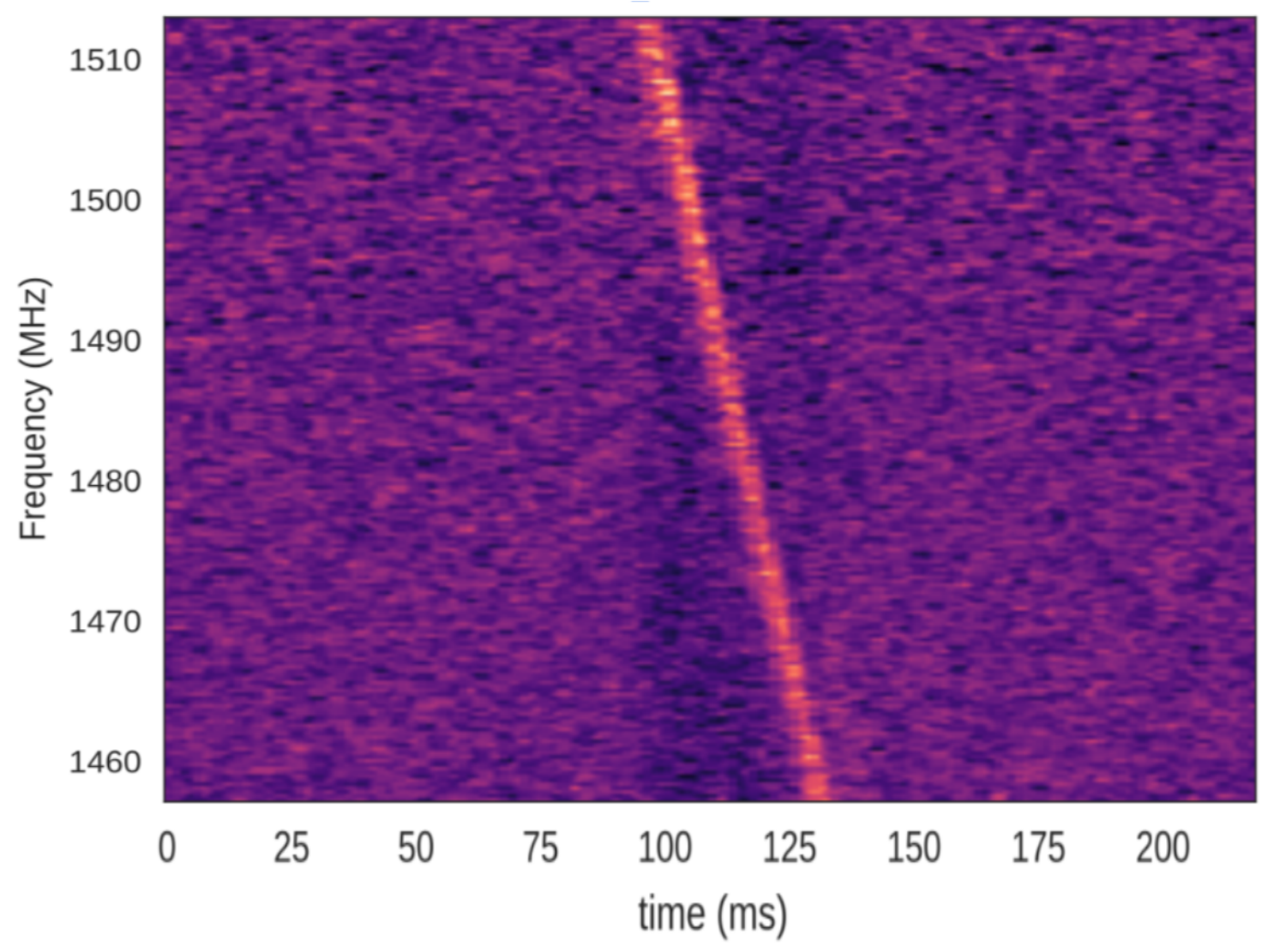

Figure 1.2 A single pulse from PSR B1900+01 observed with the ALFA (Arecibo L-band Feed Array) receiver. The horizontal axis represents time and the vertical axis shows the sky frequency with brighter colors corresponding to higher intensity. Here, the radio pulses at $1510 \mathrm{MHz}$ arrived about $25 \mathrm{~ms}$ earlier than the $1460 \mathrm{MHz}$ pulse. The DM for this pulsar is calculated to be $245 \mathrm{~cm}^{-3} \mathrm{pc}$. 
we can write

$$
\mathrm{DM}_{\mathrm{E}}=\mathrm{DM}_{\mathrm{IGM}}+\left(\frac{\mathrm{DM}_{\text {Host }}}{1+z}\right)
$$

where $\mathrm{DM}_{\mathrm{IGM}}$ is the IGM contribution and $\mathrm{DM}_{\text {Host }}$ is the host galaxy contribution of the DM (which can be estimated if we assume a similar free electron distribution for the host galaxy to that in the Milky Way). For a source with $\operatorname{redshift}^{1} z$, the $(1+z)$ factor accounts for the cosmological time dilation of the source. From this equation, it is possible to set the upper limit for $\mathrm{DM}_{\mathrm{IGM}}$ from the observation. For example, if we detect a burst at $\mathrm{DM}=360 \mathrm{~cm}^{-3} \mathrm{pc}$ and if according to NE2001 model, the DM contribution from our Galaxy is about $40 \mathrm{~cm}^{-3} \mathrm{pc}$ in that direction ${ }^{2}$, then $\mathrm{DM}_{\mathrm{E}}=320 \mathrm{~cm}^{-3}$ pc. For the purposes of this example let us assume that we can localize this FRB to a host galaxy of redshift of $z=0.32$. To estimate the $\mathrm{DM}_{\text {Host }}$, we use the result of Rane (2017) who found that average contribution from a source in a galaxy similar to the Milky Way is $110 \mathrm{~cm}^{-3}$ pc. From the equation above, in order to find $\mathrm{DM}_{\mathrm{IGM}}$, we should subtract the host contribution term from the $\mathrm{DM}_{\mathrm{E}}$. In this example, we find $\mathrm{DM}_{\mathrm{IGM}}=236 \mathrm{~cm}^{-3} \mathrm{pc}$.

\subsubsection{Scattering}

As discussed below (§1.1.6), the pulse shapes for FRBs are typically represented as a single symmetric component. Some, however, have an exponential tail. This phenomenon has long been known for radio pulsars and was first introduced

\footnotetext{
${ }^{1}$ FRB redshifts are discussed in more detail in Section 1.1.7 below.

${ }^{2}$ Note that the NE2001 model does not include a Galactic halo contribution, so in this specific example we assume that the burst is detected in the direction of Galactic Plane.
} 
by Scheuer (1968). In the model, the turbulent medium through which the radio waves propagate is modeled as a "thin screen" of irregularities located midway between the source and the observer. This screen causes small deviations in the radio wave's path, which results in a distribution of arrival times for rays that arrive at the Earth. As shown for FRB 110220 in Fig. 1.3, the amount of broadening increases with decreasing frequency. For the thin-screen model, as shown by Scheuer (1968), the $1 / e$ time constant of the broadened pulse, known as the "scattering time-scale" $(\tau)$, is proportional to $\nu^{-4}$. Note that, although scattering is produced by the same free electrons that cause the dispersion, this delay is different from the $\nu^{-2}$ delay caused by dispersion. Scattering is strongly depending on frequency, direction in the sky, and distance of the source. To quantify this, in a similar way to DM, we define scattering measure

$$
\mathrm{SM} \equiv \int_{O}^{S} C_{n_{e}}^{2} d \ell,
$$

where $C_{n_{e}}$ is the fluctuation strength along the line of sight. Further details of this property can be found in Macquart \& Koay (2013).

As described in detail by Zhu et al. (2018), FRB scattering is a powerful probe to study turbulence in the IGM and in other galaxies. A key point to stress here is that the overall level of scattering is typically low for FRBs. Naively, based on what is known from pulsars where there is a correlation between the scattering time and DM (see, e.g., Lorimer \& Kramer, 2004), we might expect significant scattering tails in the profiles of FRBs given their large DMs. The lack of scattering can be understood in terms of a lever-arm effect where, from geometrical considerations, 


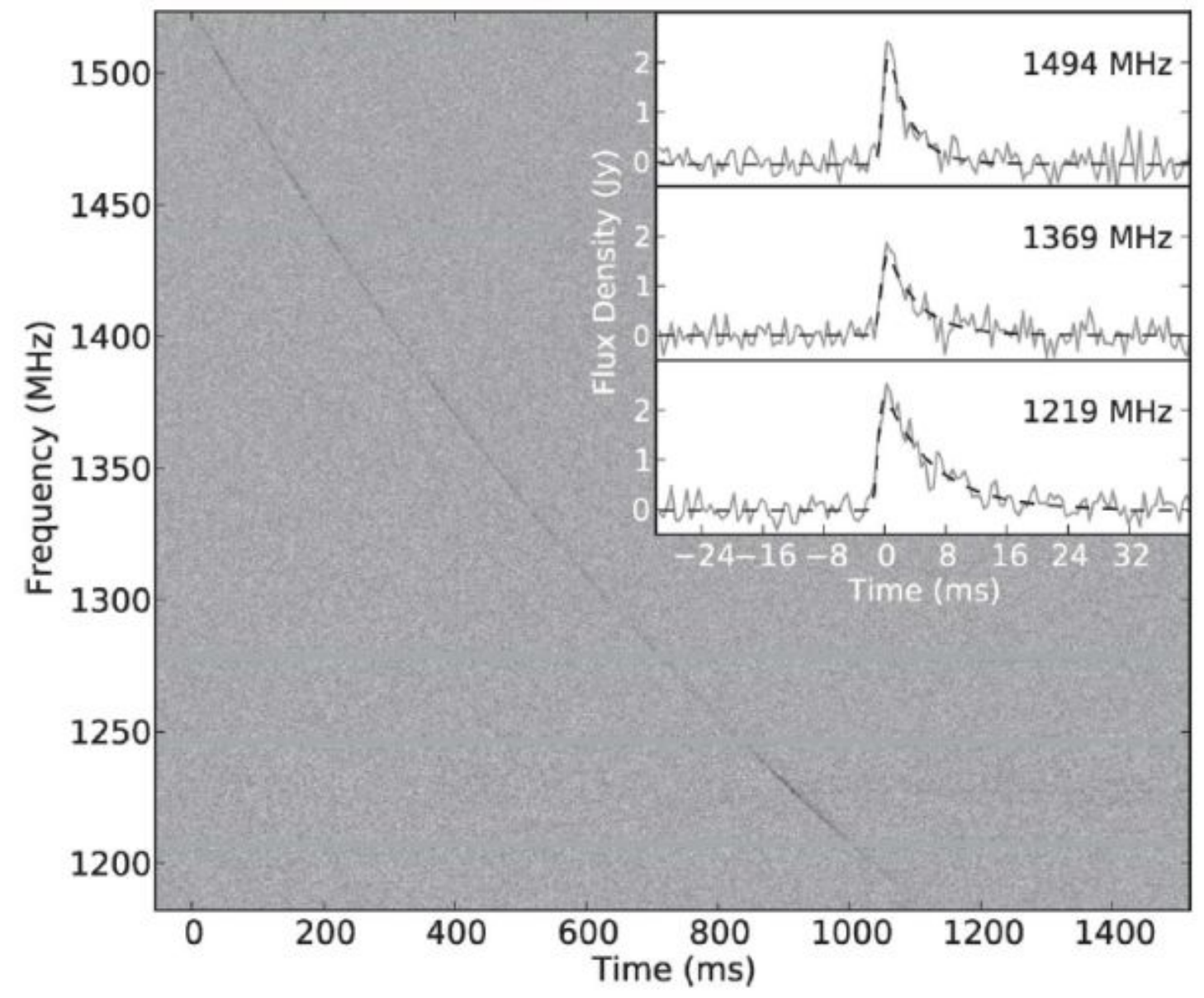

Figure 1.3 Scattering effect in FRB 110220. The main panel demonstrates the dynamic spectrum of the burst dispersed in the frequency range. The inset shows the de-dispersed pulse for three sub-bands from which we see that the burst is broader at lower frequencies compared to higher frequencies. Taken from Thornton et al. (2013). 

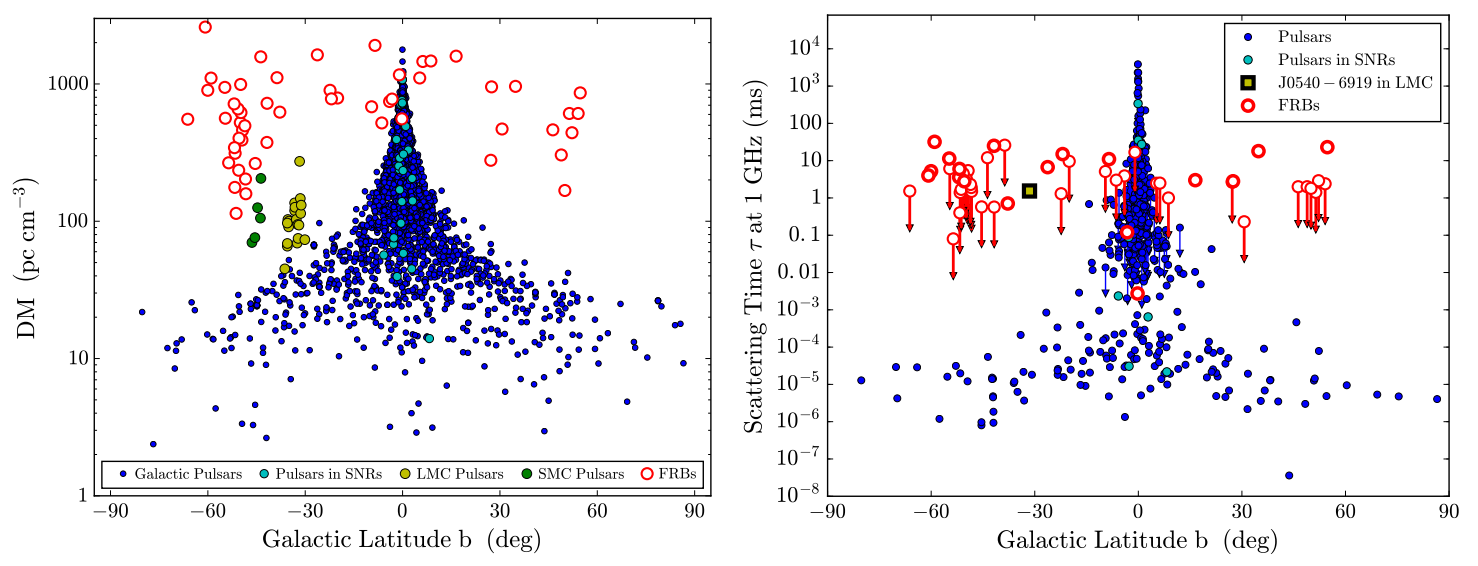

Figure 1.4 Left: DMs plotted against Galactic latitude for pulsars and FRBs. Different symbols are used for Galactic pulsars, Galactic pulsars associated with supernova remnants, pulsars in the Large Magellanic Cloud (LMC) and Small Magellanic Cloud (SMC), and FRBs. Right: Scattering times for pulsars and FRBs at $1 \mathrm{GHz}$ plotted against Galactic latitude. Taken from Cordes \& Chatterjee (2019).

scattering from contributions close to the source and observer are minimized (see, e.g., Williamson, 1972; Lorimer et al., 2013). The left-hand panel of Fig. 1.4 shows DMs plotted against Galactic latitude (b) for FRBs and pulsars in the Milky Way and in the Magellanic Clouds (LMC, SMC). Outside of the Galactic plane, DMs of FRBs are much larger than the outer envelope for Galactic pulsars. The right-hand panel of Fig. 1.4 demonstrates scattering times $\tau$ scaled to $1 \mathrm{GHz}$ versus Galactic latitude (b) for FRBs and pulsars. Pulsar scattering times span more than ten orders of magnitude compared to FRBs, which is consistent with FRB scattering occurring primarily from extragalactic gas.

\subsubsection{Scintillation}

Scintillation can be considered as the analog of optical star twinkling for compact objects at radio frequencies. Using the same framework to understand scat- 
tering, scintillation can be modeled assuming the intervening medium to be a thin screen of inhomogeneous and highly turbulent irregularities, placed midway between the pulsar and the observer. The phase perturbations produced by such a screen are correlated over a scintillation bandwidth. Unlike scattering, scintillation can increase or decrease the intensity of the signals, depending on destructive or constructive interference when the waves come back together. Therefore this effect might allow us to detect FRBs that are below our detection threshold if the medium was homogeneous. Fig. 1.5 shows the scintillation traces in the dynamic spectrum of FRB 170827 (Farah et al., 2018). Because there is little scattering expected from the Milky Way along this line of sight and the IGM, the scintillation is most likely due to screens close to the source, as thought to be the case for FRB 110512 (Masui et al., 2015). However, it is not clear how prevalent this is.

\subsubsection{Polarization}

Electromagnetic waves consist of an electric field vector and a magnetic field vector orthogonal to each other. The angle between the incidence plane and the electric field vector, known as the polarization angle, rotates as the radio wave propagates through the ionized medium. This phenomenon is referred to as 'Faraday rotation' and the change in polarization angle is proportional to the observed wavelength, with the constant of proportionality known as the rotation measure $(\mathrm{RM})$. In addition to being able to measure the total intensity of the incoming sig-

nal, most radio antennas are sensitive to polarization state of the radio signals and 


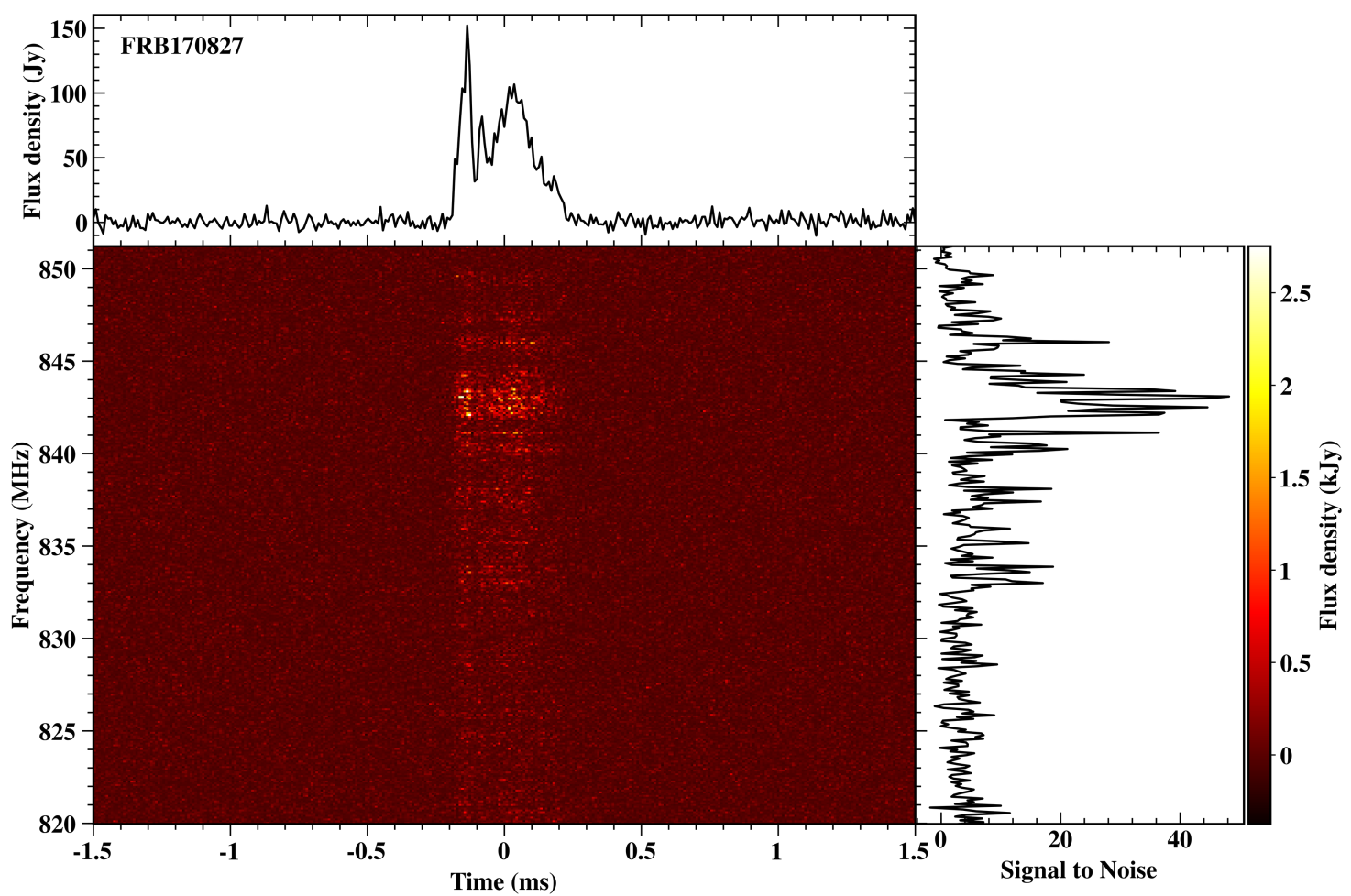

Figure 1.5 The dynamic spectrum of FRB 170827. In the heat map there is a region of enhanced emission between 840 and $845 \mathrm{MHz}$, which corresponds to the spiky peaks on the time-integrated spectrum plot on the right panel. The upper panel shows the time series profile with three major peaks. Taken from Farah et al. (2018). 
can measure Faraday rotation if they are equipped with two orthogonally oriented probes. Polarization information can be used to constrain the source's emission mechanism and to understand the medium between the source and the observer. For radio waves traveling through an ionized and magnetized medium, we define

$$
\mathrm{RM}=-0.81 \int_{O}^{S} B_{\|} n_{e} d \ell
$$

where $B_{\|}$is the component of magnetic field parallel to the line of sight and, as before, $n_{e}$ is the free electron number density. RM measurements can provide useful information about the local medium around the FRBs. A positive RM means that the magnetic field of the source is towards the observer. Combining equations 1.2 and 1.4 , for a constant $n_{e}$, we see that $\left\langle B_{\|}\right\rangle$, the average value of $B_{\|}$over the line of sight, can be found from the ratio of RM/DM. Note that this will be a poor approximation if $n_{e}$ varies.

In a similar manner to that in which polarization observations of pulsars previously helped in understanding the configuration of the magnetic field of the Galaxy (Noutsos, 2012), polarization information from the observed FRBs can be potentially used as a natural probe of the large-scale magneto-ionic content of the universe. Akahori \& Ryu (2010) and Akahori \& Ryu (2011) investigated the rotation measure (RM) due to the intergalactic magnetic field using structure formation simulations based on turbulence dynamo in the large-scale structure of the universe (Ryu et al., 2008). They predicted a log-normal probability distribution function for $|R M|$ values on large scales through filaments of galaxies in the universe. Later, Ravi et al. 
(2016) implemented the results of cosmological structure formation simulations and examined the linearly polarized, low-RM, high-intensity FRB 150807 and concluded the magnetization in the circumburst plasma is negligible and set an upper limit to the net magnetization of the cosmic web along this line of sight to be less than $21 \mathrm{nG}$. In the future, as further FRBs are found with well-measured polarimetric properties, an ensemble analysis of a sample of several hundred sources could be used to probe the origin of extragalactic magnetic fields (Vazza et al., 2018).

\subsubsection{Pulse profiles}

As shown in Fig. 1.6, FRB pulses are generally simple in form with a single component. The pulse width, usually quantified by the full-width at half maximum intensity, is currently observed to be in the range 1-34 ms. In a very similar way to radio pulsars (see, e.g., Lorimer \& Kramer, 2004), using light travel time arguments, $R=w c$, where $R$ is the radius of the source, $c$ is the speed of light, and $w$ is the pulse width. This implies a compact emitting region of size $<10,000 \mathrm{~km}$.

It is important to point out that the observed width is an upper bound to the intrinsic pulse duration due to instrumental and/or propagation effects. To quantify this, for a top-hat pulse, the observed pulse width is defined as a quadrature sum of the intrinsic pulse width, the sampling time, broadening due to dispersion, and scattering. In this case, the observed width

$$
W=\sqrt{t_{\mathrm{int}}^{2}+t_{\mathrm{samp}}^{2}+t_{\mathrm{DM}}^{2}+t_{\mathrm{DM}_{\mathrm{err}}}^{2}+\tau^{2}} .
$$




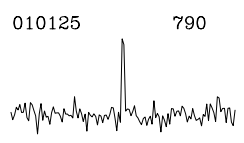

$110214 \quad 169$

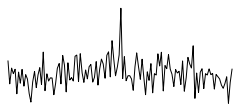

$120127 \quad 553$

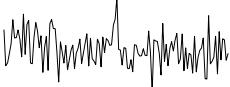

130729

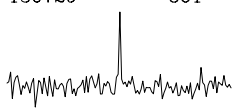

150418

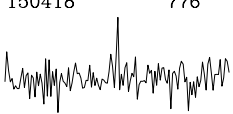

151230

960

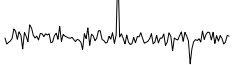

180309

263

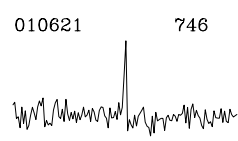

$110220 \quad 944$
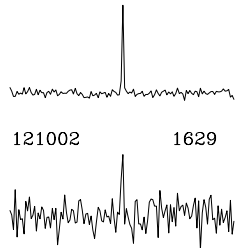

$131104 \quad 779$

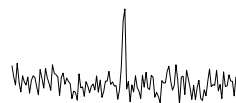

$150610 \quad 1594$

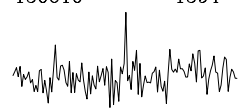

$160102 \quad 2596$

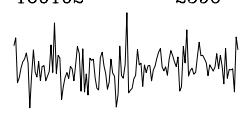

$180311 \quad 1576$

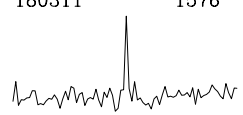

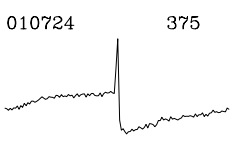
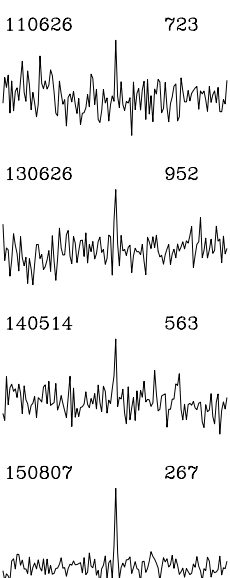

$171209 \quad 1458$

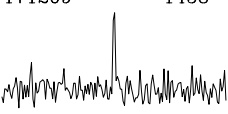

180714

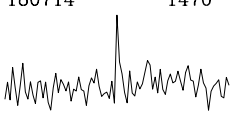

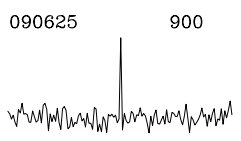

$110703 \quad 1104$

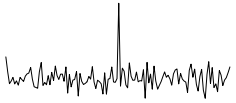

$130628 \quad 470$

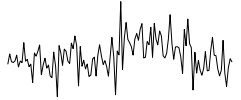

$150215 \quad 1106$

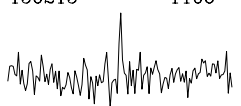

$151206 \quad 1910$

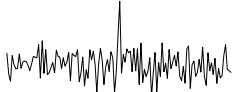

$180301 \quad 520$

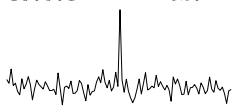

$180923 \quad 548$

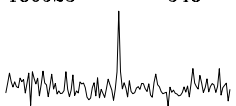

Figure 1.6 Pulse profiles from the first twenty-eight FRBs discovered using the Parkes telescope. The detections are arranged in order of date. Each pulse profile is 2 seconds long. The observed DM of each FRB is listed to the right of each pulse. Taken from Cordes \& Chatterjee (2019). 
Here $t_{\text {int }}$ is the intrinsic pulse width before intra-channel smearing and scattering broaden the pulse, $t_{\text {samp }}$ is the sampling time, and $\tau$ is the scattering timescale. The frequency-dependent dispersion delay time $\left(t_{\mathrm{DM}}\right)$ can be derived from

$$
t_{\mathrm{DM}}=8.3 \mu s\left(\frac{\mathrm{DM}}{\mathrm{cm}^{-3} \mathrm{pc}}\right)\left(\frac{\Delta \nu}{\mathrm{MHz}}\right)\left(\frac{\nu}{\mathrm{GHz}}\right)^{-3}
$$

for a bandwidth $\Delta \nu_{\mathrm{MHz}}$, an observing frequency $\nu_{\mathrm{GHz}}$. As defined by Cordes \& McLaughlin (2003), $t_{\text {DMerr }}$ is the second-order correction time to the DM smearing. Optimal detection needs the DM smearing to be removed from the measured signal by compensating time delays. This process is known as "de-dispersion" and will be explained in Section 3.3.2. This optimized DM value that is in error by an amount $\mathrm{DM}_{\mathrm{err}}$, causes the time delay $t_{\mathrm{DM}_{\mathrm{err}}}=t_{\mathrm{DM}}\left(\mathrm{DM}_{\mathrm{err}} / \mathrm{DM}\right)$.

As an example calculation, for FRB 090625 which was discovered in the 1.4GHz High Time Resolution Universe (HTRU) high-latitude survey at Parkes (Champion et al., 2016), the intrinsic pulse width, $t_{\text {int }}$, can be estimated from the observed width $W=4.4 \mathrm{~ms}$ by using the values $t_{\mathrm{DM}}=1.3 \mathrm{~ms}, \tau=3.7 \mathrm{~ms}, t_{\mathrm{samp}}=0.064 \mathrm{~ms}$, and assuming negligible $t_{\text {DMerr }}$ into Eq. 1.5. Doing this, we find $t_{\text {int }}<2.0 \pm 0.7 \mathrm{~ms}$.

\subsubsection{Redshifts}

In astronomy, redshift $(z)$ refers to the fractional change in wavelength of spectral lines received from a celestial object. In a non-relativistic case, for a line observed with a wavelength $\lambda_{\text {obs }}$ compared to a rest-frame wavelength $\lambda_{\text {int }}$, we may 
write

$$
z=\frac{\lambda_{\mathrm{obs}}-\lambda_{\mathrm{int}}}{\lambda_{\mathrm{int}}} \Longrightarrow 1+z=\frac{\lambda_{\mathrm{obs}}}{\lambda_{\mathrm{int}}}
$$

For cosmological sources, this effect is dominated by the expansion of the Universe between emission and reception so that $z \geq 0$.

Like pulsars, FRBs have no detectable spectral lines. Direct measurements of the redshifts to them can only be made via robust associations with host galaxies. At the time of writing, only five FRBs have well localized host galaxies from which redshifts can be determined. FRB 121102 was localized to a star-forming region in a dwarf galaxy with $z=0.19$, using follow-up observations with the Arecibo telescope (Chatterjee et al., 2017). Besides FRB 121102, four other single FRBs were recently localized using interferometry.

The Australian Square Kilometre Array Pathfinder (ASKAP) interferometer was able to localize FRB 180924 to a position $4 \mathrm{kpc}$ from the center of a luminous galaxy (Bannister et al., 2019). This sub-arcsecond localization allowed them to identify the massive host galaxy with $z=0.3214$. FRB 190523 was detected with the Deep Synoptic Array ten-antenna prototype (DSA-10), which consists of 4.5-m radio dishes separated by $6.75 \mathrm{~m}$ to $1300 \mathrm{~m}$, located at the Owens Valley Radio Observatory. This FRB was also localized to a few-arcsecond region containing a single massive galaxy at $z=0.66$, which is the likely host galaxy of the burst (Ravi et al., 2019). Most recently, (Marcote et al., 2020) used the European Very Long Baseline Interfereometry Network to localize FRB 180916.J0158+65, to a starforming region in a massive nearby spiral galaxy with $z=0.0337$. 
From a redshift measurement, we can make a number of inferences on the FRBs. Changing from wavelengths to frequencies in the above expression, if a pulse in the source frame is emitted at frequency $\nu_{\text {int }}$ and the observer sees the pulse at frequency $\nu_{\mathrm{obs}}$, then we can write

$$
\nu_{\mathrm{obs}}=\frac{\nu_{\mathrm{int}}}{1+z}
$$

Since frequency is inversely proportional to time, then we may write the observed pulse width

$$
W_{\mathrm{obs}}=W_{\mathrm{int}}(1+z)
$$

where $W_{\text {int }}$ is the intrinsic pulse width. Using these expressions and ignoring broadening due to propagation effects, we see that an FRB observed at $1.4 \mathrm{GHz}$ with $z=0.3$ with a width of $3 \mathrm{~ms}$ was emitted at $1.8 \mathrm{GHz}$ with a width of $2.3 \mathrm{~ms}$.

To infer distances from redshifts, we need to invoke a cosmological model. A number of distance metrics are in use and we refer the reader to Hogg (1999) for a useful overview. One commonly used definition is to take coordinates which "comove" with the expanding Universe. This so-called comoving distance

$$
D(z)=\frac{c}{H_{0}} \int_{0}^{z} \frac{d z^{\prime}}{\sqrt{\Omega_{m}\left(1+z^{\prime}\right)^{3}+\Omega_{\Lambda}}}
$$

where $c$ is the speed of light, $H_{0}$ is the Hubble constant $\left(68 \mathrm{~km} \mathrm{~s}^{-1} \mathrm{Mpc}^{-1}\right.$ from Chen \& Ratra (2011)), $\Omega_{m}$ is the total energy density of matter and $\Omega_{\Lambda}$ is the total energy density of dark matter (Zheng et al., 2014). While the above integral can 

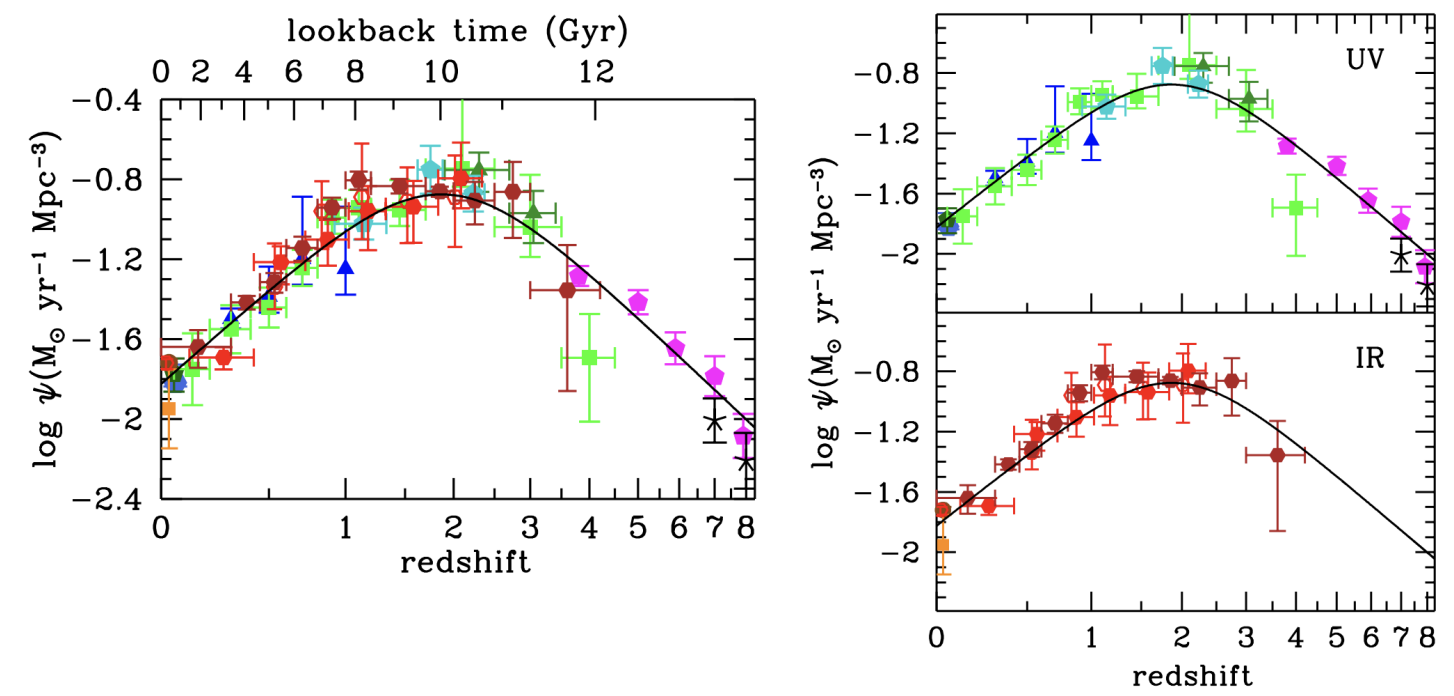

Figure 1.7 The history of cosmic star formation from (top right panel) far ultraviolet surveys, (bottom right panel) infrared surveys, and (left panel) combined far ultra-violet and infrared rest-frame measurements. The solid curve in the three panels plots the best-fit star-formation rate distribution in Equation 1.11. Figures taken from Madau \& Dickinson (2014).

not generally be solved analytically, it is straight forward to evaluate numerically. As an example, for $z=0.5$, we find ${ }^{3}$ a co-moving distance of 1.9 Gpc.

The true distribution of FRB redshifts is not well constrained at present. It is anticipated, however, that they might closely follow the star formation rate $\psi(z)$ which can be fit (Madau \& Dickinson, 2014) to the following redshift-dependent function:

$$
\psi(z)=0.015 \frac{(1+z)^{2.7}}{1+\left(\frac{1+z}{2.9}\right)^{5.6}} M_{\odot} \mathrm{yr}^{-1} \mathrm{Mpc}^{-3}
$$

This function is shown along with the observational constraints from different surveys in Fig. 1.7, demonstrating the function peaking around $z=2$.

\footnotetext{
${ }^{3}$ using this online tool: http://www.astro.ucla.edu/ wright/CosmoCalc.html
} 


\subsubsection{Energetics}

A key parameter required to confront different theoretical explanations for FRBs is a measure of their intrinsic luminosity, $L$, which is inferred from the observed pulse intensity recorded at the telescope and some measure of distance. Assuming FRBs as discrete sources, we can define the source flux density $\left(S_{\nu}\right)$ as the power received by the telescope of unit collecting area at some frequency, $\nu$. Unlike brightness (also known as specific intensity), flux is not an intrinsic property of a source and it depends on the distance of the source from the observer. The peak flux density $\left(S_{\text {peak }}\right)$ is the maximum intensity in the pulse profile. For FRBs, it is also common to quote the integrated pulse energy or fluence

$$
\mathcal{F}=S_{\text {peak }} W_{\text {eq }}
$$

where $W_{\text {eq }}$ is the so-called equivalent width of the pulse, defined to be the width of a top-hat pulse with the same area as the area under the observed pulse profile. Typical units of flux density are Janskys, where $1 \mathrm{Jy}=10^{-26} \mathrm{~W} \mathrm{~m}^{-2} \mathrm{~Hz}^{-1}$. Expressing pulse widths in ms usually leads to fluences being quoted in the hybrid units of Jy ms.

Luminosity is typically defined as the total power radiated at the source over some range of frequencies. A common simplifying strategy invoked for FRBs (see, e.g., Petroff et al., 2019) is to assume a flat spectral dependence, i.e., that $S_{\nu}$ is 
independent of frequency. In this case, the isotropic equivalent luminosity

$$
L=4 \pi D_{L}^{2} S_{\nu} \Delta \nu
$$

where $\Delta \nu$ is the observed bandwidth and $D_{L}$ is the luminosity distance. This latter quantity, which is different from the comoving distance introduced earlier, can be thought of the distance a source of a given luminosity would result in an observed flux in a flat non-expanding Universe. It can be shown (see, e.g., Hogg, 1999) that

$$
D_{L}=(1+z) D(z)
$$

where, as before, $D(z)$ is the comoving distance and $z$ is the redshift.

Inserting commonly used units into Eq. 1.13, and making use of the approximation $D_{L} \simeq 2 z(z+2.4)$ Gpc which is valid for $z<1$ (Petroff et al., 2019), we find

$$
L \simeq 4.5 \times 10^{34} \mathrm{~W}\left(\frac{S_{\text {peak }}}{\mathrm{Jy}}\right)\left(\frac{\Delta \nu}{100 \mathrm{MHz}}\right)\left(\frac{z^{2}(z+2.4)^{2}}{1+z}\right) .
$$

As an example, for a 1 Jy pulse observed over a $100 \mathrm{MHz}$ band from FRB 121102 (with $z=0.19$; Chatterjee et al., 2017), we find $L=9.2 \times 10^{33} \mathrm{~W}$.

Although typical radio sources are powered by non-thermal processes (Condon \& Ransom, 2016, for a review, see, e.g.), it is often useful to compute the so-called 'brightness temperature' $\left(T_{b}\right)$ which is is the thermodynamic temperature of a black body of equivalent luminosity. Since radio wavelengths are in the Rayleigh Jeans 
regime, it can be shown (see, e.g., Lorimer \& Kramer, 2004) that

$$
T_{b}=\frac{S_{\nu} d^{2}}{2 k \nu^{2} W^{2}}
$$

where $k$ is Boltzmann's constant and $W$ is the pulse width. Inserting units into this expression, we find

$$
T_{b} \simeq 10^{36} K\left(\frac{S_{\mathrm{peak}}}{\mathrm{Jy}}\right)\left(\frac{\nu}{\mathrm{GHz}}\right)^{-2}\left(\frac{W}{\mathrm{~ms}}\right)^{-2}\left(\frac{D_{L}}{\mathrm{Gpc}}\right)^{2}
$$

As an example, for a $5 \mathrm{~ms}$ pulse observed at $1 \mathrm{GHz}$, with the flux density of $1 \mathrm{Jy}$ from a source $100 \mathrm{Mpc}$ away, the brightness temperature is almost $10^{38} \mathrm{~K}$. This is clearly far too high to be caused by thermal processes, like pulsars; the emission properties demand a non-thermal emission mechanism.

To describe the number of FRBs per unit luminosity interval, some form of luminosity function is generally invoked and constrained using on the results of various surveys. For example, using a sample of FRBs from Parkes surveys, Luo et al. (2018) applied the Schechter function (Schechter, 1976), in which the number of FRBs per unit logarithmic luminosity interval

$$
\phi(\log L) d \log L=\phi^{*}\left(\frac{L}{L^{*}}\right)^{\alpha+1} e^{-\frac{L}{L^{*}}} d \log L
$$

where $\phi^{*}$ is a normalization factor and $\alpha$ and $L^{*}$ are free parameters. Under different assumptions for the host galaxy type, the authors used a Bayesian-based Monte Carlo approach to naturally account for the detectability of FRBs. They found the 
power law index range $-1.8<\alpha<-1.2$ and the cut-off luminosity $L^{*} \simeq 2 \times 10^{37} \mathrm{~W}$. It is important to point out that the form of the distribution is generally limited by survey sensitivities. Future surveys will be able to better probe the faint end of the luminosity function.

\subsubsection{Source counts and event rates}

Source counts represent a less model-dependent way to characterize the FRB population and can be used to readily make predictions for future surveys. To see how this works, consider an object of luminosity $L$ emitting isotropically at some distance $D$. The observed flux density $S=L /\left(4 \pi D^{2}\right)$. Now, for a population of these objects uniformly distributed in space with some density $\rho_{0}$, the number of objects within a distance $D, N(<D)=\frac{4}{3} \rho_{0} \pi D^{3}$. Combining these two expressions to eliminate distance, and ignoring constant factors, we see that $N(>S) \propto S^{-3 / 2}$. This source count function assumes a population of standard candles in a Euclidean universe and is a useful starting point for discussing the population of FRBs and is often parameterized by $N(>S) \propto S^{-\alpha}$ where $\alpha$ is known as the source count index. For a population of bursting sources like FRBs, since the event rate $(\mathcal{R})$ is directly proportional to the number of sources, we may also write $\mathcal{R}\left(>S_{\min }\right) \propto S_{\text {min }}^{-\alpha}$ and use event rates from a survey with some limiting flux density for detection, $S_{\text {min }}$. Alternatively, since fluence $\mathcal{F} \propto S$, we may also write $\mathcal{R}\left(>\mathcal{F}_{\text {min }}\right) \propto \mathcal{F}_{\text {min }}^{-\alpha}$ for some survey fluence limit $\mathcal{F}_{\text {min }}$. As we discuss in Chapter 4, current observational constraints imply a significantly flatter distribution with $\alpha<0.8$. 
Table 1.1 summarizes the FRB event rate constraints for different analysis methods and surveys. Assuming a volume within $z=1$, and a fluence limit of 1 Jy ms (Thornton et al., 2013), the equivalent volumetric rate is large and of order $10^{3}$ FRBs per $\mathrm{Gpc}^{3}$ per year. This is already an order of magnitude larger than the total GRB rate for the same redshift range (Frail et al., 2001), and it is important to note that the FRB rate has not been corrected for beaming effects.

Table 1.1 Published FRB rates, $(\mathcal{R})$. For each entry, in addition to the relevant survey frequency, we also provide the percentage confidence intervals (CI) and fluence $\operatorname{limit}\left(\mathcal{F}_{\lim }\right)$.

\begin{tabular}{cccccc}
\hline \hline $\mathcal{R}$ & $\begin{array}{c}\text { Range } \\
\text { FRBs/sky/day }\end{array}$ & CI & $\begin{array}{c}\mathcal{F}_{\text {lim }} \\
\text { Jy ms }\end{array}$ & $\begin{array}{c}\text { Frequency } \\
\text { MHz }\end{array}$ & Reference \\
\hline$\sim 225$ & - & - & 6.7 & 1400 & (Lorimer et al., 2007) \\
10000 & $5000-16000$ & 68 & 3.0 & 1400 & (Thornton et al., 2013) \\
4400 & $1300-9600$ & 99 & 4.4 & 1400 & (Rane et al., 2016) \\
7000 & $4000-12000$ & 95 & 1.5 & 1400 & (Champion et al., 2016) \\
3300 & $1100-7000$ & 99 & 3.8 & 1400 & (Crawford et al., 2016) \\
587 & $272-924$ & 95 & 6.0 & 1400 & (Lawrence et al., 2017) \\
1700 & $800-3200$ & 90 & 2.0 & 1400 & (Bhandari et al., 2018) \\
37 & $29-45$ & 68 & 37 & 1400 & (Shannon et al., 2018) \\
\hline
\end{tabular}

Making an analogy with radio pulsars, which have similar pulse widths to FRBs, the implied beaming fraction from pulse profiles studies is about 0.1 (see, e.g., Tauris \& Manchester, 1998) which would boost the true volumetric rate of FRBs by a further factor of 10. In a recent analysis, Ravi (2019) also pointed out this discrepancy and noted that the FRB rates exceed essentially all reasonable estimates of the cataclysmic progenitor rates. This is somewhat reminiscent of the so-called birth-rate problem for millisecond pulsars (see, e.g., Kulkarni \& Narayan, 1988) where the pulsar rate initially exceeded that of their proposed progenitors, the low-mass X-ray binaries. In recognition of this problem, Ravi (2019) proposes 
that if all FRBs repeat, then the implied rate of FRBs would be substantially less.

\subsection{Historical development of the field}

\subsubsection{The Lorimer Burst (FRB 010724)}

In 2007, the very first highly dispersed, bright radio burst was found accidentally in an archival pulsar survey targeting the Small Magellanic Cloud (SMC) using the Parkes radio telescope (Lorimer et al., 2007). The burst was $5 \mathrm{~ms}$ long and its estimated peak flux density was $30 \mathrm{Jy}$. Its high dispersion measure (DM) of $375 \mathrm{~cm}^{-3}$ pc was strongly implying an extragalactic origin for the source, since the Galactic DM contribution along the line of sight of the burst according to the NE2001 was only $12 \%$ of the total measured value. Soon this pulse became known as the "Lorimer burst", which now is known as FRB 010724. Fig. 1.8 shows the dynamic spectrum (radio frequency versus time domain) for the burst.

The extraordinary brightness and the implied extragalactic distance, among the celestial, rather than terrestrial origin of the Lorimer burst was in agreement with possible scenarios on extragalactic radio signals caused from supernovae (Colgate, 1975; Colgate \& Noerdlinger, 1971) or from exploding black holes (Rees, 1977; Phinney \& Taylor, 1979).

The discovery of this burst suggested a new class of radio transients with unknown emission mechanism and raised the question about how rare these events are. Moreover, finding similar events and identifying their host galaxies could result in probing the ionized intergalactic medium and determining the baryonic content 


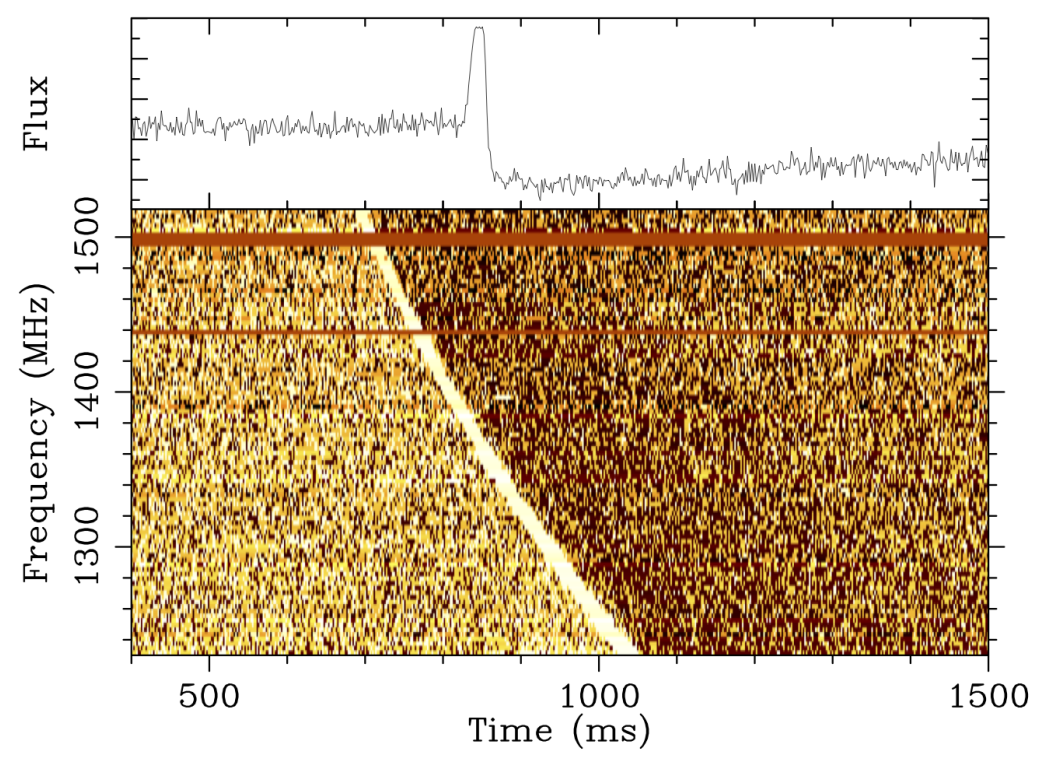

Figure 1.8 (Lorimer et al., 2007) seen by the Parkes multibeam radio telescope (taken from Cordes \& Chatterjee (2019)). The horizontal axis shows time with a resolution of $1 \mathrm{~ms}$. The top panel shows flux density of the burst (in arbitrary units) summed across all the frequency channels. The dip in the baseline right after the peak is due to the high intensity of the source which saturated the detector. The bottom panel shows the 'dynamic spectrum' of the burst. The quadratic sweep of this dispersed pulse and the pulse broadening towards lower frequencies are easily distinguished with the bright colors across the band. The signal was also detected in three other neighboring beams, out of the total thirteen beams of the receiver. The two red horizontal lines at $1440 \mathrm{MHz}$ and $1500 \mathrm{MHz}$ are frequency channels that have been zapped since they were corrupted by radio frequency interference.

of the universe (Ioka, 2003; Yang \& Zhang, 2016).

\subsubsection{Perytons}

Three years after publication of the Lorimer burst, pulses with a similar frequency sweep in the dynamic spectra were reported by Burke-Spolaor et al. (2011). This work detailed 16 pulses appearing in all beams of the multibeam system, each with a duration of about ten milliseconds. Even though the frequency-swept emission of these events was mimicking the dispersion of an astrophysical pulse, the 
authors distinguished these detections from the original Lorimer burst with the name 'perytons', after the mythical Greek elk that casts a human shadow, implying these sources were masquerading as FRBs.

Several other detections of perytons were published in subsequent years (Kocz et al., 2012; Bagchi et al., 2012; Saint-Hilaire et al., 2014). While it was generally accepted that these were not a celestial phenomenon, the terrestrial origin of perytons was first confirmed in 2015 when Petroff et al. (2015) reported three more perytons at Parkes with a real-time detection system that was used to demonstrate that they were produced by signals from on-site microwave ovens. Petroff et al. (2015) also showed that the microwaves on site could not have caused the Lorimer burst and therefore it stood out as a prototype of a new celestial phenomenon.

\subsubsection{The Keane Burst (FRB 010621)}

The burst, now known as FRB 010621 and shown in Fig. 1.9, was discovered in the data from the Parkes Multibeam Pulsar Survey (PMPS; Manchester et al., 2001) in a re-analysis designed to find Rotating Radio Transients (RRATs) and Lorimertype bursts (Keane et al., 2011, 2012). Originally named as J1852-08, FRB 010621 was detected with a best-fit DM of $745 \pm 10 \mathrm{~cm}^{-3}$ pc. Assuming the NE2001 model (Cordes \& Lazio, 2002) is correct, considering the Milky Way maximum DM contribution of $533 \mathrm{~cm}^{-3}$ pc from the line of sight towards the source, they concluded that the burst is probably extragalactic. Dedispersing the entire band based on the estimated DM resulted in a pulse width of $7.3 \mathrm{~ms}$. 


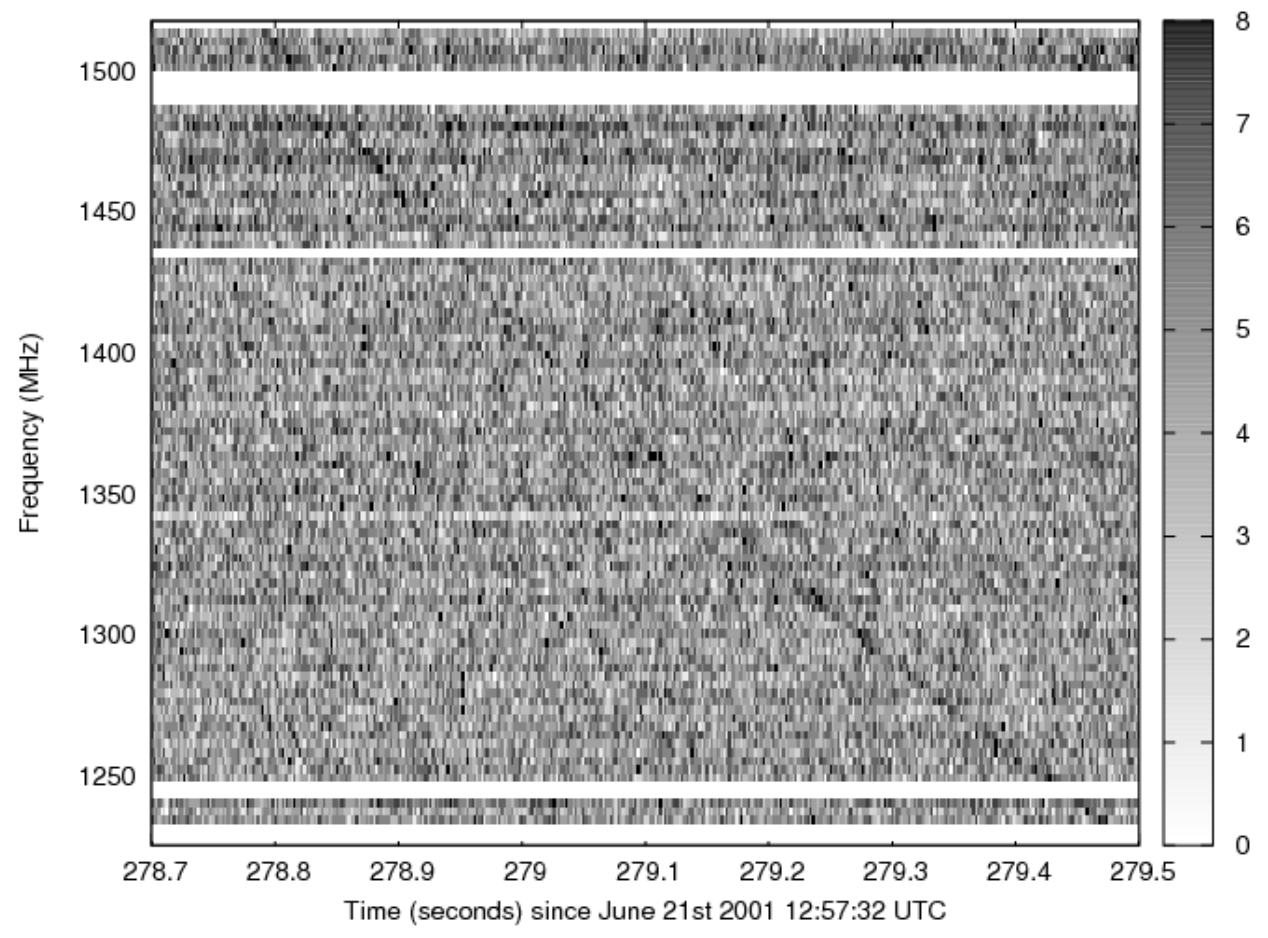

Figure 1.9 Waterfall plot showing FRB 010621. Here, the grey scale represents the amplitude. Nine of the 96 frequency channels were flagged as being spoiled by radio frequency interference and have been set to zero. Taken from Keane et al. (2012). 
Later, Bannister \& Madsen (2014) set new distance constraints by studying the Keane burst's line of sight in order to determine a more precise electron density measurement. Using a Monte Carlo simulation, and the observed DM, they concluded with $90 \%$ confidence that this burst was from a Galactic source along an extremely dense line of sight and the distance is $14 \pm 6 \mathrm{kpc}$. It was not possible to differentiate between the two possible scenarios proposed by Keane et al. (2012), namely a pulsar with an unusual amplitude distribution or an annihilating black hole.

\subsubsection{Thornton Bursts (FRBs 110220, 110627, 110703 and 120127)}

Thornton et al. (2013) reported the detection of four millisecond-duration bursts, all detected in the high Galactic latitude region of the High Time Resolution Universe (HTRU) survey which was designed to detect pulsars and other radio transients (Champion et al., 2016). This was the first time the term "fast radio burst" was coined and they were labelled in YYMMDD format: FRB 110220, FRB 110627, FRB 110703, FRB 120127. Like Lorimer et al. (2007), the authors concluded that these four FRBs have cosmological redshifts in the range $0.5<z<1.0$. Note that these are inferred, and not directly measured, redshifts. No X-ray or gamma-ray counterparts that possibly could be associated with the bursts were detected. After the detection of these four "Thornton bursts" and despite what has been previously suggested by Bannister \& Madsen (2014) on the Keane burst, the original Lorimer burst and Keane Burst were not unique events anymore and represented a new 
population of objects of unknown cosmological origin.

\subsubsection{The first repeating source, FRB 121102}

Up to this point, all detected FRBs were assumed to be one-off events due to the fact that follow-up observations to detect additional bursts at the same DM and sky position failed. This apparent non-repeating nature of these bursts has led to the suggestion that they originate from cataclysmic events (Falcke \& Rezzolla, 2014). In 2016, Spitler et al. (2016) reported observations of ten additional bursts from the direction of FRB 121102, which was originally discovered in an on-going large-scale survey for pulsars using the Arecibo telescope (Spitler et al., 2014). These additional bursts had the same DMs and sky positions as the original burst. This discovery brought into question the cataclysmic nature of FRBs, at least for FRB 121102. Scholz et al. (2016) reported six additional radio bursts from FRB 121102: five with the Green Bank Telescope at $2 \mathrm{GHz}$, and one at $1.4 \mathrm{GHz}$ with the Arecibo telescope. They also presented X-ray observations using Chandra and Swift telescopes and archival optical/IR observations from WISE (Wide-field Infrared Survey Explorer) and IPHAS (INT Photometric H-Alpha Survey) but none of these observations shows an obvious counterpart to FRB 121102. They concluded that it is extremely unlikely that FRB 121102 is Galactic, since any nebula that could provide the observed dispersion should be visible in VLA, WISE, and/or IPHAS observations. Therefore, the certain extragalactic distance and repeating nature of FRB 121102 lead us to favor an origin for the bursts that invokes a young extragalactic neutron 
star.

Chatterjee et al. (2017) were able to localize FRB 121102 using high-timeresolution radio interferometric observations. This sub-arcsecond localization showed that FRB 121102 is located within 100 mas of a faint persistent radio source with a faint optical counterpart. Later, studies of FRB 121102, allowed the burst to be localized to a star-forming region in a host dwarf galaxy with $z=0.19$ (Chatterjee et al., 2017), using the VLA acting jointly with the Arecibo telescope. This redshift measurement made this the first FRB with a direct distance determination (Tendulkar et al., 2017). Follow-up studies showed a large and variable rotation measure towards this source, suggesting that FRB 121102 is in an extreme and dynamic magneto-ionic environment. A neutron star origin is consistent with both such an environment and the short burst durations (Michilli et al., 2018). See the details of the detection in Fig. 1.10 and the details of the localization in Fig. 1.11.

\subsubsection{A second repeating source (FRB 180814.J0422+73)}

A second repeating FRB, 180814.J0422+73, was recently discovered by CHIME (Canadian Hydrogen Intensity Mapping Experiment CHIME/FRB Collaboration et al., 2019a). CHIME consists of four 100-m by 20-m semi-cylinders in British Columbia, Canada. The telescope cannot be steered; instead it observes the sky overhead as the Earth turns. The CHIME collaboration reported the detection of six repeat bursts from FRB 180814.J0422+73 among 13 detected FRBs during the telescope's pre-commissioning phase (CHIME/FRB Collaboration et al., 2019b). All 

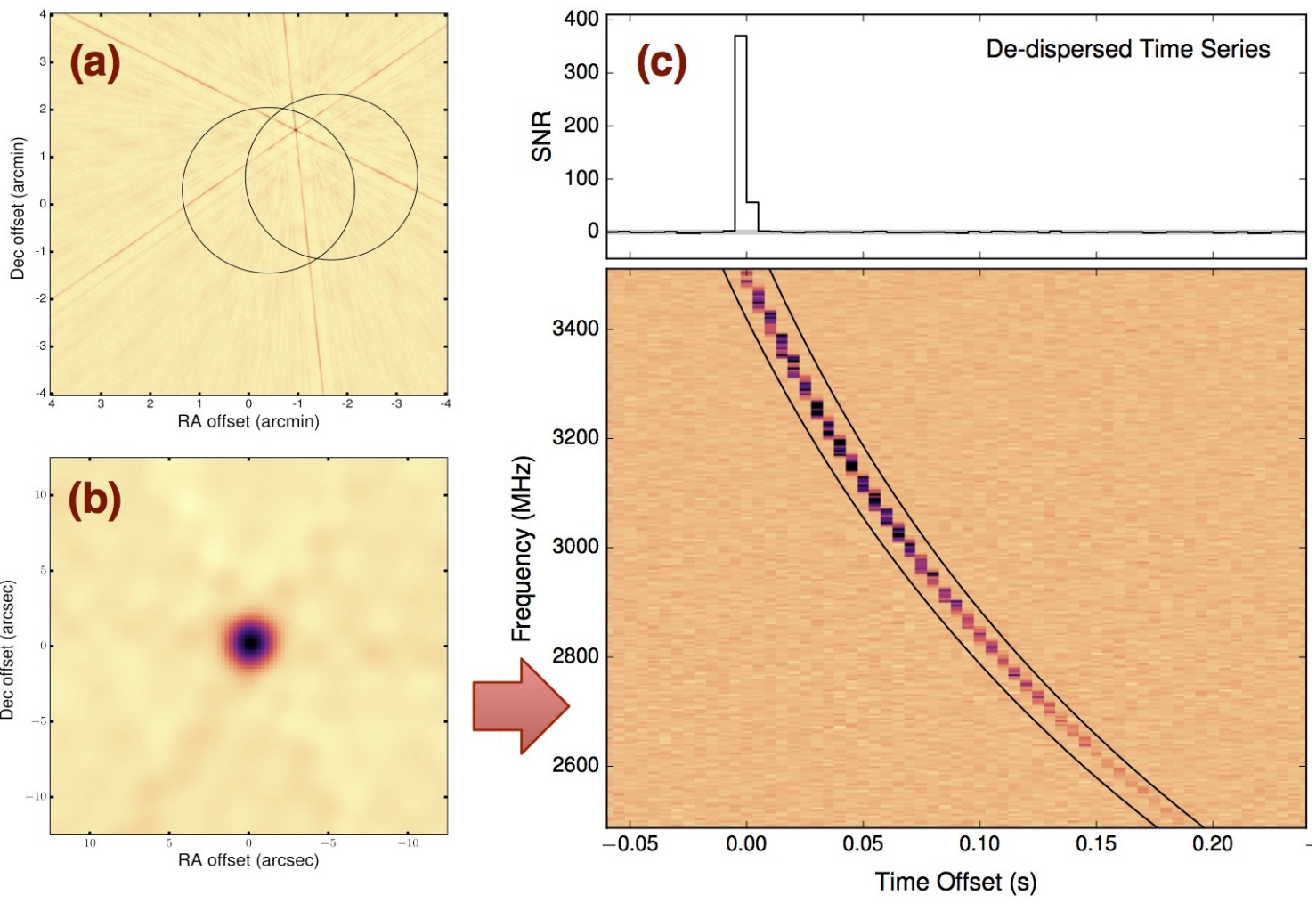

Figure 1.10 (a) A 5-ms dispersion-corrected image showing a burst from FRB 121102. The overlapping circles show the approximate localization uncertainty from previous Arecibo detections. (b) A zoomed in portion of the above image, de-convolved and re-centered on the detection, showing the 0.100 localization of the burst. (c) The bottom panel shows the waterfall plot extracted from phased VLA visibilities at the burst location shows the $\nu^{-2}$ dispersive sweep of the burst. The solid black lines illustrate the expected sweep for DM $=558 \mathrm{~cm}^{-3} \mathrm{pc}$. The top panel shows the de-dispersed light curve. Taken from Chatterjee et al. (2017). 


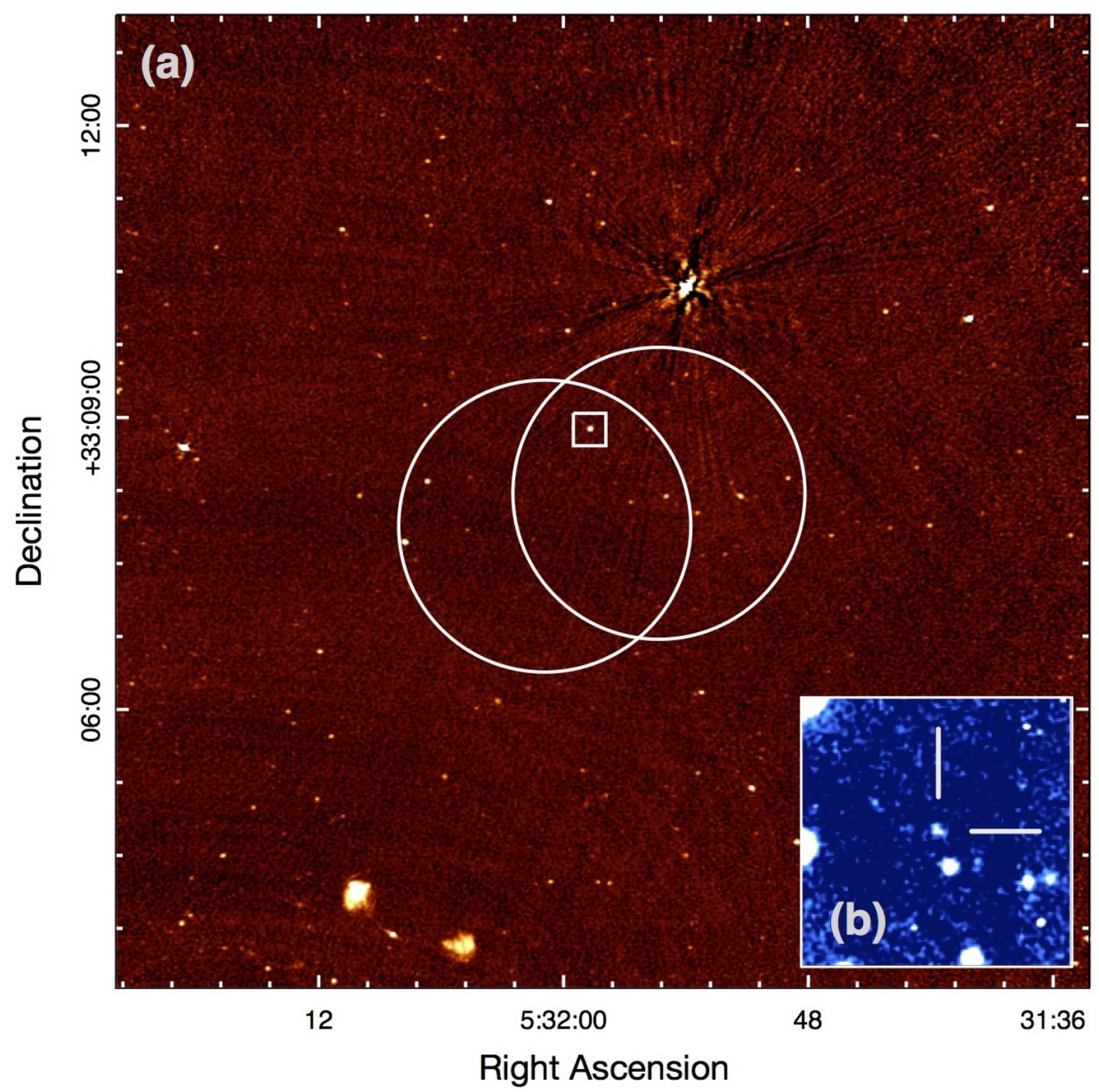

Figure 1.11 (a) VLA image at $3 \mathrm{GHz}$ with a combination of array configurations. The image resolution is 2 arcseconds. The overlapping white circles show the Arecibo detection uncertainty regions (30" beam FWHM). The 20 arcsecond white square shows the radio counterpart of the bursts detected at the VLA. (b) Gemini r-band image of the 20 arsecond square shows an optical counterpart, as identified by the 5 arcsecond bars. Taken from Chatterjee et al. (2017). 
these repeat bursts had the same dispersion measure (DM) of $\sim 189 \mathrm{~cm}^{-3} \mathrm{pc}$, which is roughly twice the Galactic DM distribution along that line of sight. They verified there are no cataloged Galactic foregrounds that could provide an extra DM contribution and placed an upper limit on the source redshift of $z=0.11$, corresponding to a distance of $\sim 500 \mathrm{Mpc}$.

\subsubsection{Localized non-repeating FRBs}

In addition to FRB 180924 localized which was localized by ASKAP (See Section 1.1.7), FRB 190523 was localized to a few-arcsecond region containing a single massive galaxy at $z=0.66$, which is the likely host galaxy of the burst (Cordes \& Chatterjee, 2019). It was detected with the Deep Synoptic Array ten/antenna prototype (DSA-10), which consists of 4.5-m radio dishes separated by $6.7 \mathrm{~m}$ to $1300 \mathrm{~m}$, located at the Owens Valley Radio Observatory.

\subsubsection{More Repeating FRBs}

So far, only one of the ASKAP FRBs has been shown to repeat. Kumar et al. (2019) recently reported the discovery of faint bursts from FRB 171019. Green Bank Telescope (GBT) observations showed bursts that are almost 600 times fainter than the original ASKAP detection and indicate that some FRBs could be exhibiting much fainter repeat pulses that might not be probed currently by the discovery instruments.

Most recently, CHIME discovered eight repeating FRBs, varying from two re- 
peating bursts to ten (The CHIME/FRB Collaboration et al., 2019). These eight repeating FRBs probably represent the bright and/or high-rate end of the repeating FRB distribution. The DM distributions of these eight repeaters are indistinguishable from the previous non-repeating FRBs detected by CHIME. The variety of these recently detected repeaters compared to non-repeating CHIME FRBs detected previously is suggestive of different environmental properties (e.g. larger burst widths and complex morphologies and downward-drifting sub-bursts in several events) and emission mechanisms for the repeating FRBs. One of the sources has a very low RM compared to FRB 121102, which suggests that not all repeaters share the environmental properties of the first repeater. These properties are included in Table 1.2, and waterfall plots of the bursts are shown in Fig. 1.12. As mentioned above, one of these repeaters, FRB 180916.J0158+65, was recently localized to a nearby massive spiral galaxy (Marcote et al., 2020) and a new study reported the detection of a $16.35 \pm 0.18$ day periodicity for this FRB (Amiri et al., 2020). Their results disfavour purely random processes scenarios and suggest a mechanism for periodic modulation either of the burst emission itself, or through external amplification or absorption.

\subsection{Thesis Outline}

The rest of this thesis is organized as follows. In Chapter 2, I briefly discuss the main FRB models that have been suggested so far and classify them into different progenitors. In Chapter 3, I give a summary of FRB surveys carried out so far, as 


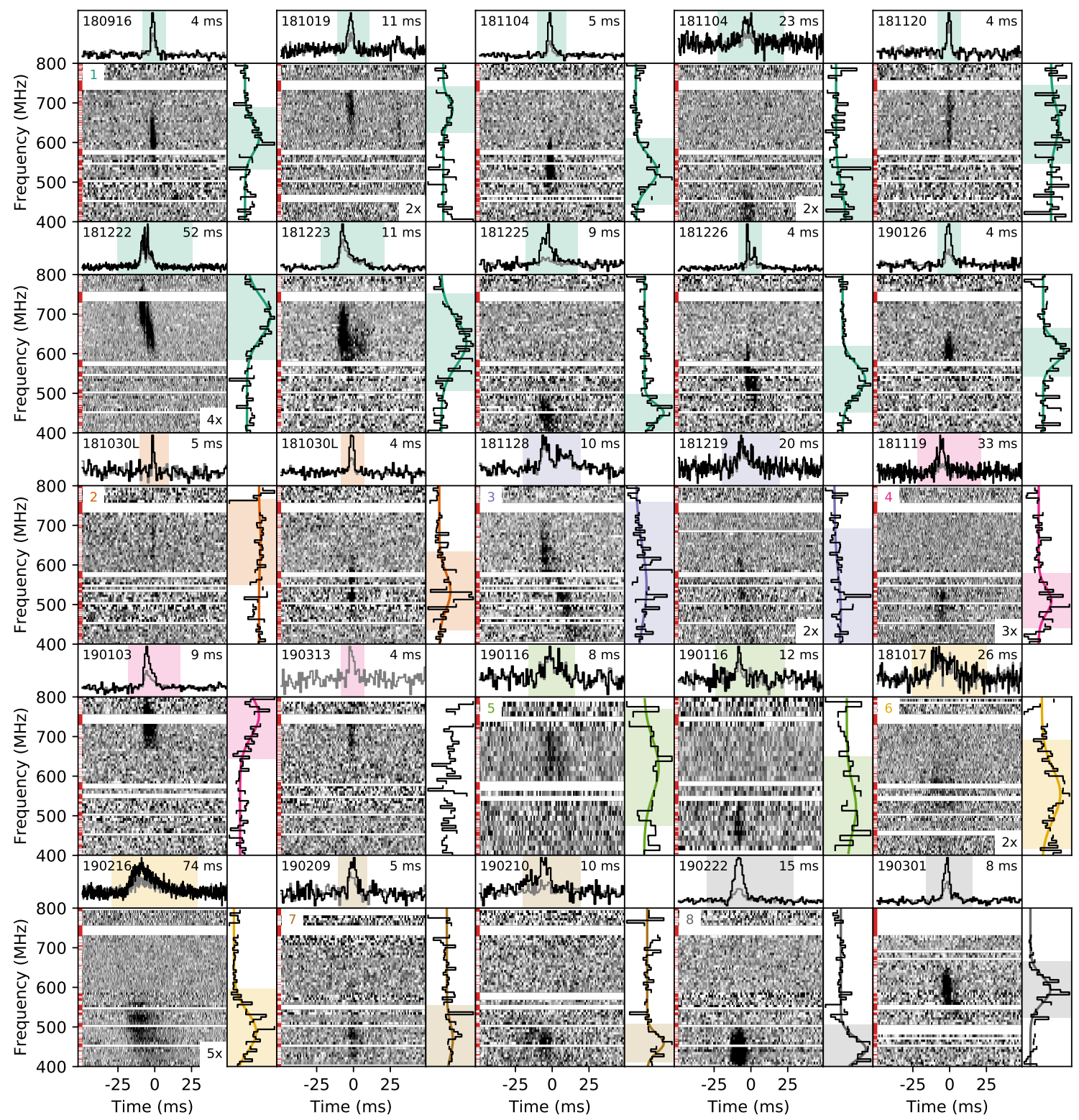

Figure 1.12 Frequency versus time plots of the bursts listed in Table 1.2. Each panel shows the de-dispersed data along with the integrated pulse profile on top and the on-pulse spectrum on the right. Windows show 100 time samples ( $\sim 100 \mathrm{~ms})$, unless indicated otherwise by the multiplicative factor in the bottom right corner. Pulse widths are in the top right corner. The shaded region in the profile (four times the pulse width) was used for the extraction of the on-pulse spectrum. The shaded region in the on-pulse spectrum shows the full width at tenth maximum (FWTM) of a Gaussian fit, except for the third bursts of Source 4. Underlying missing or masked channels of the full-resolution intensity data are depicted by red lines on the left of the intensity data. Taken from The CHIME/FRB Collaboration et al. (2019). 
Table 1.2 Properties of the repeating sources including eight new CHIME/FRB repeating bursts. The naming convention (YYMMDD.JHHMMDD) was used in the current absence of a final naming convention agreed upon by the community. $\mathrm{DM}$ column is the weighted average $\mathrm{DM}, \mathrm{DM}_{\mathrm{NE2001}}$ is the maximum model prediction along this line-of-sight for the NE2001 model (Cordes \& Lazio, 2002), and $N_{\text {bursts }}$ is the number of repeating bursts detected at the time of writing. The last column is the fluence completeness limits based on their faintest detected burst.

\begin{tabular}{ccccc}
\hline Name & $\begin{array}{c}\mathrm{DM} \\
\left(\mathrm{pc} \mathrm{cm}^{-3}\right)\end{array}$ & $\begin{array}{c}\mathrm{DM}_{\mathrm{NE2001}} \\
\left(\mathrm{pc} \mathrm{cm}^{-3}\right)\end{array}$ & $N_{\text {bursts }}$ & $\begin{array}{c}\text { Completeness } \\
(\text { Jy ms })\end{array}$ \\
\hline 180916.J0158+65 & $349.2(3)$ & 200 & 28 & 1.0 \\
181030.J1054+73 & $103.5(3)$ & 40 & 2 & 17 \\
181128.J0456+63 & $450.5(3)$ & 110 & 2 & 4.0 \\
181119.J1200+65 & $364.05(9)$ & 34 & 3 & 2.6 \\
190116.J1249+27 & $441(2)$ & 20 & 2 & 5.7 \\
181017.J1705+68 & $1281.6(4)$ & 43 & 2 & 5.6 \\
190209.J0937+77 & $425.0(3)$ & 46 & 2 & 3.8 \\
190222.J2052+69 & $460.6(2)$ & 87 & 2 & 5.4 \\
180814.J0422+73 & $189.4(4)$ & 87 & 6 & 3.4 \\
171019 & $460.4(2)$ & 37 & 4 & 0.4 \\
121102 & $565(5)$ & 188 & $>114$ & 0.01 \\
\hline
\end{tabular}

well as the single-pulse FRB search techniques. In Chapter 4, I present the results from a commensal survey with the Green Bank 20 meter telescope. Although no new FRBs were found in this project, we were able to constrain the source count index in our analysis. In Chapter 5, I describe the results from the ALFABURST FRB survey, which commensally uses the ALFA receiver of the Arecibo telescope to search for FRBs. A number of FRB candidates from our analysis are presented, as well as the detection of a Galactic source. Finally, In Chapter 6, I summarize some ongoing results from observations using the Arecibo telescope which follow up on short-gamma ray burst sources. I conclude Chapter 6 by presenting plans for future work at both Green Bank and Arecibo. 


\section{Chapter 2}

\section{Models for FRBs}

Due to the limitation of observations carried out to date, today, over twelve years after the first FRB discovery, a self-consistent picture that describes their nature remains elusive. With the exception of only three FRBs so far, we are not able to measure the distance to them independently. This makes it challenging to set the energy budget for the source(s) of the bursts. Moreover, the mystery of whether there are two FRB populations versus one is still unresolved. At the very least, the existence of repeating FRBs means that models to explain them must be episodic in nature rather than a one-time occurence from a cataclysmic event.

Over the past decade, and at the time of writing, at least 45 published progenitor theories for the origin of FRBs have been suggested for both repeating and non-repeating FRBs (for a collection of these theories, see; Platts et al., 2018), from which some could be tested based on data from observed FRBs (for a review, see Katz (2016)). Out of the different suggested models, the extragalactic compact object scenario is the most common. To be more specific, 31 out of these 45 suggested theories invoke compact objects. In this Chapter, I group the models in the following subsections with a brief explanation for each. An up-to-date and complete list of all currently published models can be found at the FRB Theory Wiki ${ }^{1}$.

\footnotetext{
${ }^{1}$ https://frbtheorycat.org
} 


\subsection{Isolated Neutron Star Models}

\subsubsection{Giant Pulses}

Cordes \& Wasserman (2016) and Lyutikov et al. (2016) suggested that FRBs are giant pulses from extragalactic neutron stars. Connor et al. (2016) proposed, by extrapolating giant pulses similar to the Crab pulsar back to its first century, that FRBs are caused by super giant pulses from very young pulsars in supernova remnants. They posit that a change in polarization characteristics would signify a change in the magnetic field configuration, which is needed for bright pulse production. Further information about the polarization of a large sample of FRBs are needed in order to test this model.

\subsubsection{Starquakes}

The energy release accompanying starquakes produced on the surface of a neutron star have been considered as a source of repeating FRBs. Wang et al. (2018) found that the bursts of FRB 121102 are consistent with the aftershock sequence of an earthquake, where the bursts' time-decaying rate of seismic activity falls within the typical values of earthquakes. They also show that the burst energy distribution of FRB 121102 has a power law form, much like that of the Gutenberg-Richter law for earthquakes (Gutenberg \& Richter, 1956). 


\subsection{Magnetar Giant Flares}

As the strongest magnets in the present universe, 'magnetars' are a class of young and highly magnetized neutron stars that display dramatic variability at X-ray and soft gamma-ray wavelengths, ranging from a few milliseconds to major month-long outbursts. Magnetar emission is powered by the evolution and decay of an ultra-strong internal magnetic field, stressing and breaking the neutron-star crust, which in turn drives twists of the external magnetosphere and powerful magnetospheric currents (for a recent comprehensive review, see Kaspi \& Beloborodov, 2017). Models that invoke giant flares from magnetars have been discussed frequently in the literature to date. For instance, Popov \& Postnov (2010) proposed a possible relationship between FRBs and hyperflares from extragalactic soft gamma repeaters (SGR). Lyubarsky (2014) subsequently proposed that an FRB could be attributed to a strongly magnetized pulse propagating through the relativistic magnetar wind that inflates in the surrounding medium. In other words, the interaction of this magnetic pulse with the plasma within the nebula can form the bursts. Metzger et al. (2017) then showed that the properties of the host galaxy of FRB 121102 are consistent with those of long-duration gamma-ray bursts (LGRBs). This sub-class of the GRB phenomenon are thought to be extragalactic and produce long (anywhere from seconds to minutes) duration powerful jets of gamma rays generated by the collapse and explosion of a compact object.

Nicholl et al. (2017) investigated the possibility that the repeating FRBs originate from millisecond magnetars by comparing the magnetar birth rates, the FRB 
volumetric rates, and host galaxy demographics. They found that if FRB 121102 is a typical FRB source, then properties like rates, lifetime, energetics, and host galaxies for FRBs are consistent with expectations for millisecond magnetars from superluminous supernovae (SLSNe) ${ }^{2}$ and/or LGRBs. Most recently, Metzger et al. (2019) discussed the possibility of FRBs being generated from forward shocks of blast waves interacting with previously decelerated waves, such as flare ejecta from young magnetars. These flares are expected to produce prompt gamma-ray, X-ray, and possibly optical flares as well as high dispersion measure and high rotation measure radio waves. Their calculations show the production of FRBs with isotropic radiated energies in the range $10^{30-33} \mathrm{~J}$ with durations between 0.1 and $10 \mathrm{~ms}$. The deceleration of the blast wave results in a downward frequency drift structure seen in some of the repeating FRBs so far. Further observations of more repeaters could provide useful diagnostics and more decisive constraints on this model.

\subsection{Merging and Colliding Neutron Star Models}

This class of models has been commonly invoked in an attempt to explain non-repeating FRBs. For example, Totani (2013) argued against the giant flares of soft gamma-ray repeaters and suggested binary neutron star mergers as the origin of FRBs. Wang et al. (2016) proposed that the magnetic interaction between double neutron stars, one of which is highly magnetized compared to the other one, during their final inspiral within the framework of a unipolar inductor model

\footnotetext{
${ }^{2}$ Superluminous supernovae are a class of high energy stellar explosion with luminosities at least 10 times higher than that of regular supernovae
} 


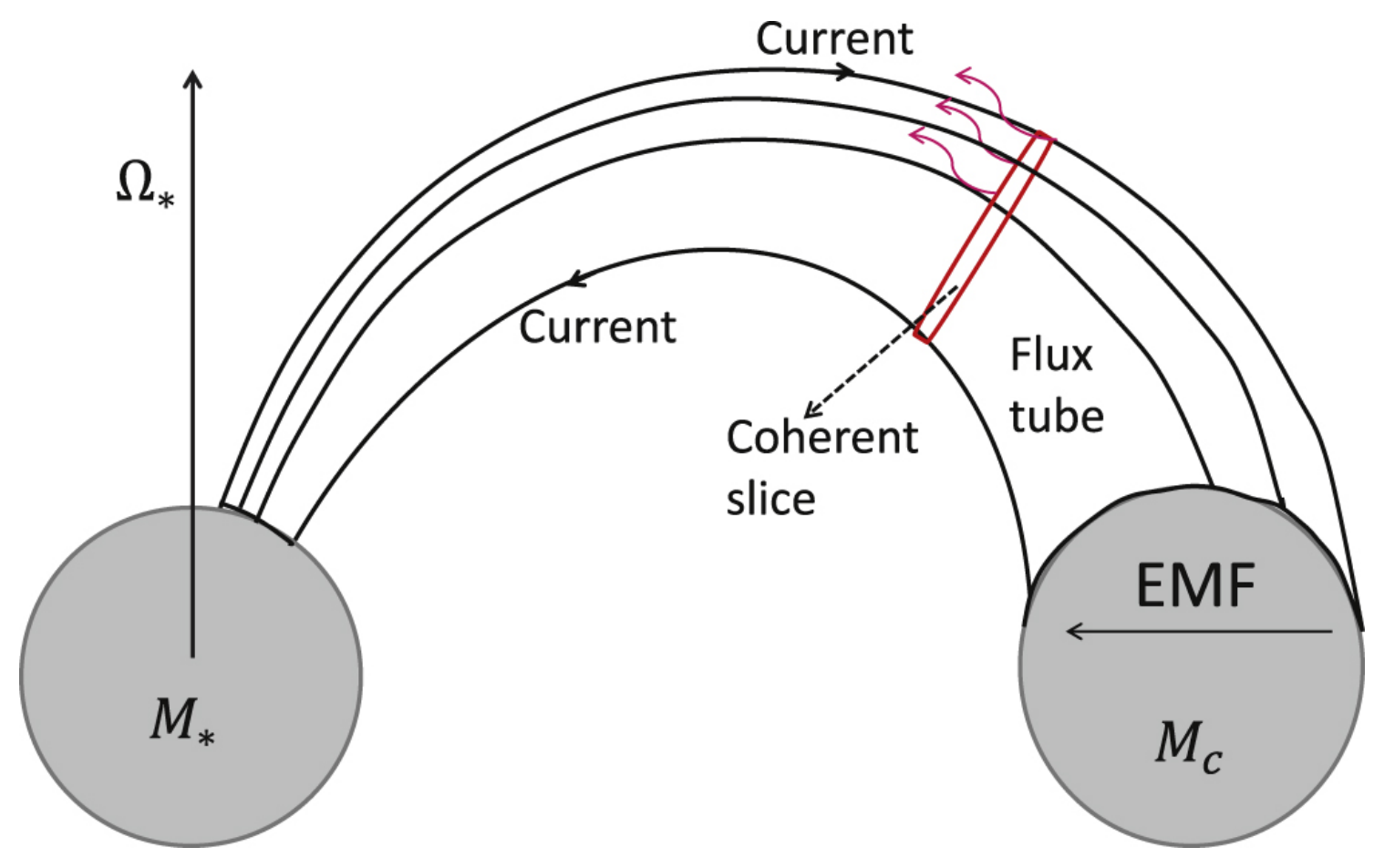

Figure 2.1 A cartoon of an electric circuit based on the unipolar inductor model during the final inspiral of double neutron stars. The red block is a slice where curvature radiation of electrons is coherent. Taken from Wang et al. (2016).

can be the origin of FRBs. The companion neutron star that was less magnetized crosses the magnetosphere of the primary highly magnetized neutron star and simultaneously produces an electromotive force, by which electrons are accelerated to ultra-relativistic speeds. Fig. 2.1 demonstrates a schematic picture of this model during the final inspiral of this binary system.

Yamasaki et al. (2018) also investigated binary neutron star mergers as a possible origin of both repeating and non-repeating FRBs. They used a generalrelativistic simulation of a binary neutron star merger to show that the ejecta happens about $1 \mathrm{~ms}$ after the merged star reaches its maximum rotation speed. 


\subsection{Collapsing Neutron Star Models}

This family of models is also common among the progenitor models for nonrepeating FRBs. One model in this family that has received a lot of attention is so-called 'blitzar'3 model, in which a neutron star collapses into a black hole and the FRB is produced from the orphaned magnetic field of the original neutron star. Falcke \& Rezzolla (2014) defined blitzars as accelerated electrons from the travelling magnetic shock dissipating a significant fraction of the magnetosphere and producing a massive radio burst. They suggested FRBs might trace the solitary and almost silent formation of high-redshift black holes with a mass between 5-100 $\mathrm{M}_{\odot}$. They also argued that the bursts could be an electromagnetic complement to gravitational wave emission and reveal a new formation and evolution scenario for black holes and neutron stars that are not seen as gamma-ray bursts.

Most et al. (2018) presented a systematic study of the gravitational collapse of a rapidly rotating and magnetized supermassive neutron $\operatorname{star}^{4}$. They found that the magnetic field lines of the neutron star must break and reconnect during merger and the electromagnetic waves propagate outside the event horizon. This will produce strong electromagnetic emission within the energetics of the observed FRBs. The relevant timescale here is the time it would take a star to collapse if the pressure supporting it against gravity were suddenly removed. To estimate this timescale using Newtonian assumptions, we can calculate the time it would take for a particle

\footnotetext{
${ }^{3}$ Blitz $($ German $)=$ lightning flash.

${ }^{4}$ This refers to a neutron star created above the theoretical mass limit as a result of spin down due to various torques, especially the magnetic dipole spin down which causes the centrifugal force to not be able to support the star anymore.
} 
to fall freely from a star's surface into its center. For a particle of mass $m$, we may write

$$
m \frac{v^{2}}{R}=\frac{G M m}{R^{2}}
$$

where $M$ is the star's mass, $R$ is the star's radius and $v$ is the velocity of the particle. Setting the dynamical timescale $t=R / v$, we find

$$
\frac{R^{2}}{t^{2}}=\frac{G M}{R^{3}}
$$

and thus:

$$
t \cong \sqrt{\frac{R^{3}}{G M}}
$$

For example, for a star with a mass of $10 \mathrm{M}_{\odot}$ and a radius of $30 \mathrm{~km}$, the dynamic timescale would be $\sim 0.1 \mathrm{~ms}$, which is in agreement with FRB pulse widths reviewed in the previous chapter.

Gupta \& Saini (2018) argued that even repeating FRBs can also be modeled within the context of a collapse framework provided that the super massive object implodes either into a 'Kerr black hole'5 surrounded by highly magnetized plasma or into a strange quark star. Unfortunately, these authors do not give details of expected observational signatures or diagnostics that could be used to distinguish this idea from other ones.

\footnotetext{
${ }^{5}$ A spinning black hole without electrical charge
} 


\subsection{Compact Object Interaction Models}

\subsubsection{Compact Object Binary Systems}

Egorov \& Postnov (2009) discussed the possibility of FRBs being the result of a supernova shock on a neutron star magnetosphere filled with plasma in a massive binary system. Kashiyama et al. (2013) introduced coalescing white dwarf binaries as the cause of FRBs, considering the coherent emission in the polar region of rapidly rotating, magnetized massive white dwarfs formed just after the merger.

Gu et al. (2016) proposed a magnetic white dwarf and a rapidly spinning neutron star with strong bipolar magnetic field binary model for the repeaters. When the white dwarf fills its Roche lobe ${ }^{6}$, violent mass transfer occurs through the first Lagrangian point $(\mathrm{L} 1)^{7}$. As a result, the accreted magnetized material may trigger magnetic reconnection when they approach the neutron star's surface, and therefore the electrons can be accelerated to ultra-relativistic speeds. An FRB can be powered by the curvature radiation of these relativistic electrons moving along the neutron star's magnetic field lines. The outward movement of the white dwarf due to the conservation of its angular momentum may dominate over the inward movement owing to the gravitational radiation, and therefore the white dwarf may be kicked away from the neutron star after a burst, and the next burst can happen when the system becomes semi-detached again through the gravitational radiation. They showed that such an intermittent Roche lobe overflow mechanism can cause

\footnotetext{
${ }^{6}$ This refers to the tear-drop-shaped region around a star in a binary system within which orbiting material is gravitationally bound to that star.

${ }^{7}$ The point where the gravitational attraction of one mass partially cancels the other mass's gravitational attraction
} 
the repeating bursts in FRBs, as the process of accretion, and thus the magnetic reconnection, may repeat. The timescale of emission is assumed to be the same as that of magnetic reconnection, and the time interval between adjacent bursts is derived from its relationship to the mass transferred by the first burst. Fig. 2.2 demonstrates a schematic sketch of this suggested model.

\subsubsection{Pulsar-orbiting Body Interactions}

Mottez \& Zarka (2014) proposed an explanation based on a pulsar-orbiting body (planet, asteroid, or white dwarf) for the origin of the bursts. They focused their analysis on the waves emitted from the magnetic wake of the body in the pulsar wind and compared it with the observations. Dai et al. (2016) and Geng \& Huang (2015) argued that the repeating FRBs are highly magnetized pulsars traveling

through asteroid belts of other stars or comets. Dai et al. (2016) suggested during each pulsar-asteroid impact, an electric field induced outside the asteroid has such a large component parallel to the stellar magnetic field that causes the electrons to tear off the asteroidal surface and accelerate instantaneously, resulting in coherent curvature radiation, observed as an FRB. Geng \& Huang (2015) suggested during the impact process, a hot plasma fireball will form. The ionized matter inside the fireball will then expand along the magnetic field lines. Coherent radiation from the thin shell at the top of the fireball will account for the observed FRBs. Istomin (2018) proposed a scenario in which the close passage of a dense body near a hot neutron star can be the origin of the bursts. 
(a)
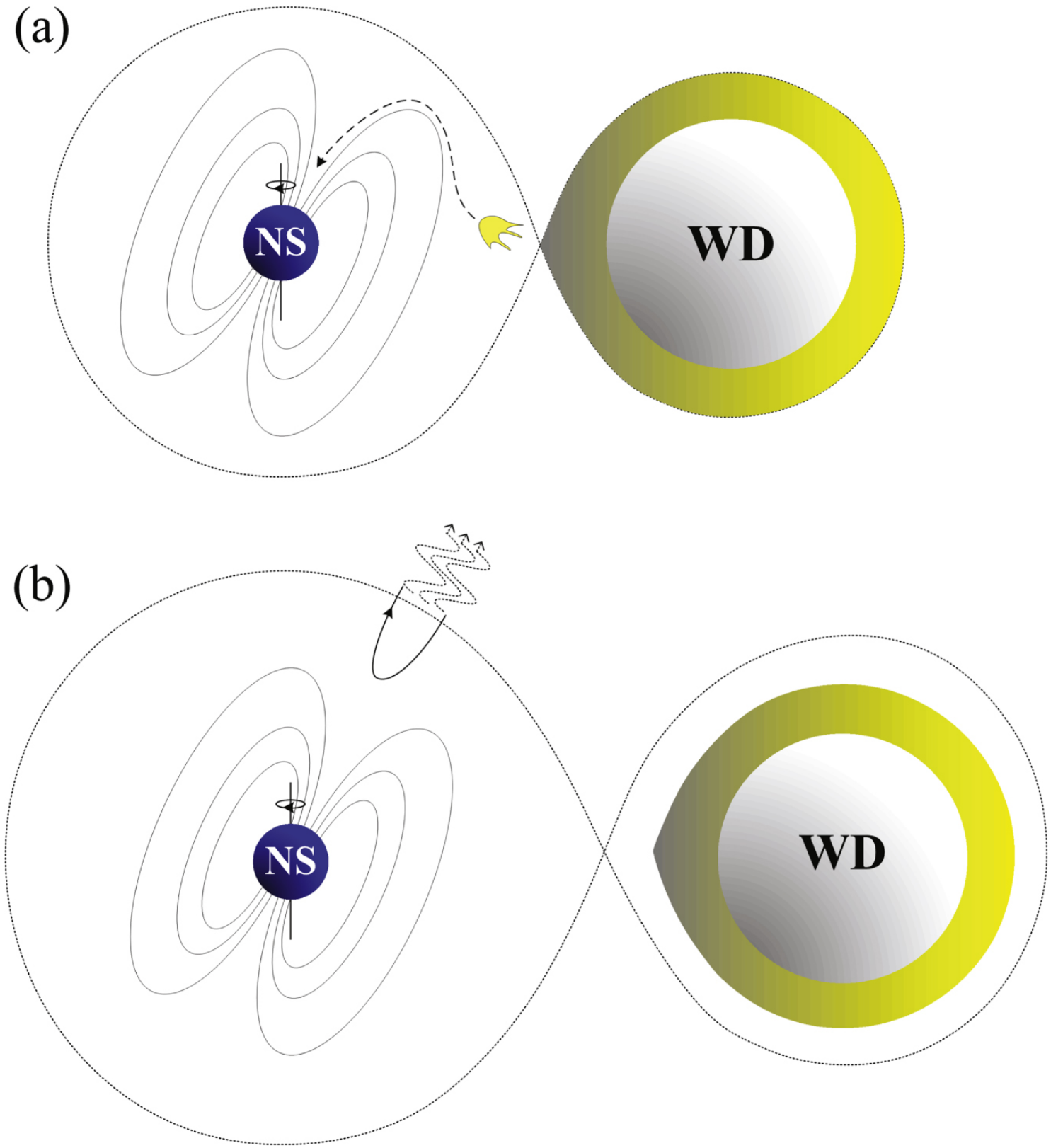

Figure 2.2 Intermittent Roche-lobe overflow in a neutron star-white dwarf (NS-WD) binary system: (a) The mass transfer occurs through the inner Lagrangian point (L1) after the white dwarf fills its Roche lobe; (b) After the mass transfer, the white dwarf is kicked away and the accreted materials trigger magnetic reconnection and strong electromagnetic radiation. Taken from Gu et al. (2016). 


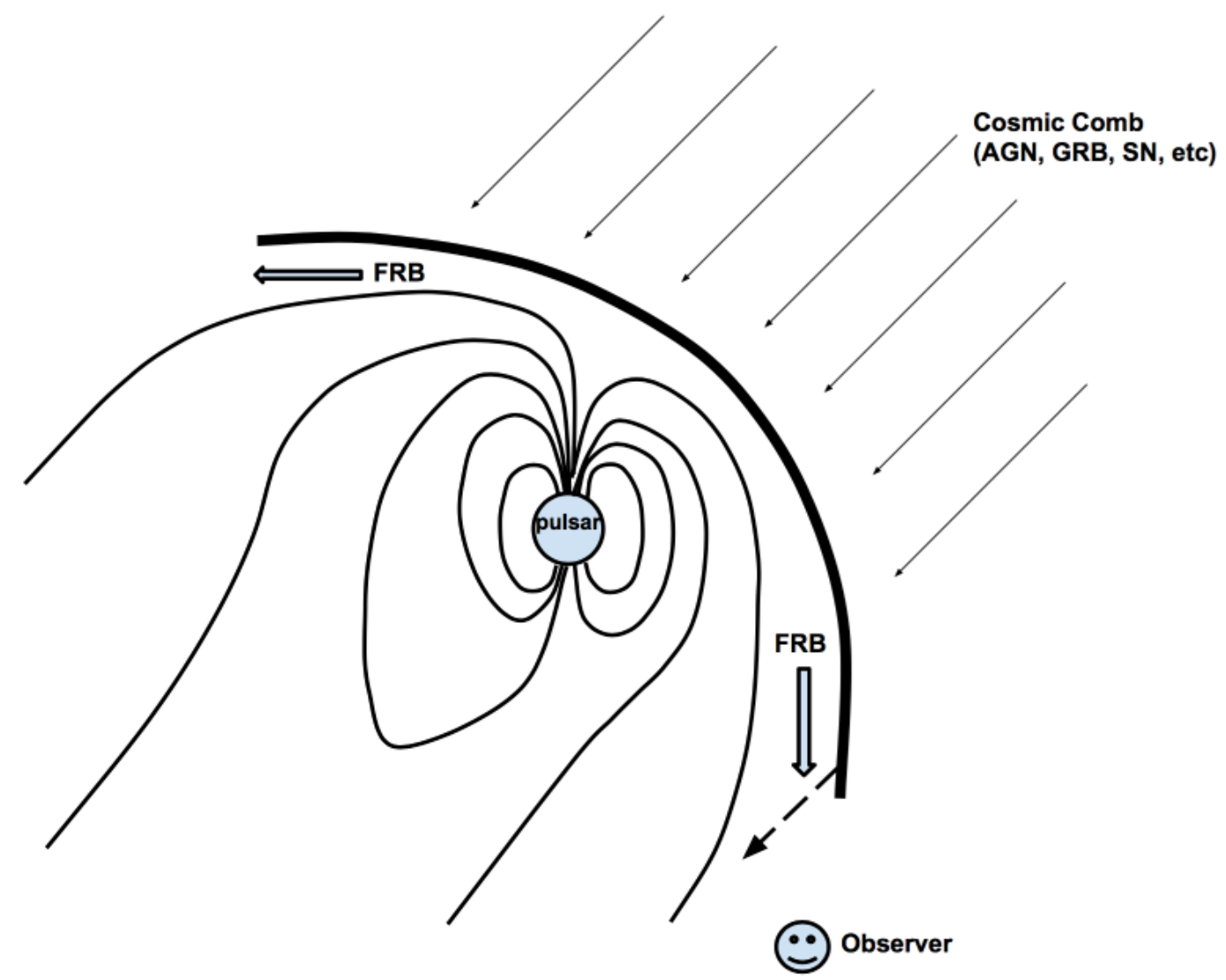

Figure 2.3 Schematic illustration of cosmic comb. An FRB is produced in the sheath region, which abruptly sweeps the line of sight. Taken from Zhang (2017).

\subsubsection{Cosmic Combs}

Dokuchaev \& Eroshenko (2017) proposed a model for FRBs due to the collisions of neutron stars in the central clusters of evolved galactic nuclei. More specifically, FRBs may be produced during the close periastron approach and at the process of the final binary merging. Zhang (2017) proposed a bright FRB can be produced by a regular, unnoticeable pulsar at a cosmological distance if its magnetosphere is abruptly "combed" by a nearby, strong plasma stream toward the anti-stream direction. A schematic illustration of this "cosmic comb" is shown in Fig. 2.3. 


\subsubsection{Dark Matter Models}

Van Waerbeke \& Zhitnitsky (2019) explored the possibility of FRBs being related to axion quark nugget $(\mathrm{AQN})$ dark matter model. This model was first proposed by Zhitnitsky (2003) as a natural explanation of the observed density ratio of the dark matter. AQNs are composite objects of standard quarks in a novel phase. In this theory, an AQN falling through a neutron stars magnetosphere may be able to produce sufficient magnetic energy for a burst. Shock waves caused by the infalling AQN would trigger magnetic reconnection, and produce a giant flare that we observe as an FRB.

\subsection{Black Hole Progenitors}

\subsubsection{Dark Matter-induced Collapses}

Fuller \& Ott (2015) explained the possibility of a neutron star capturing ambient dark matter particles as they scatter off the neutron star nucleons and become gravitationally bound. After the dark matter particles reach the neutron star temperature, they fall to the center of the neutron star. Here they accumulate until they reach a critical mass and collapse into a black hole. The black hole will then completely surround the neutron star, ejecting the neutron star magnetosphere, causing violent magnetic reconnection. The resultant coherent curvature radiation may be consistent with a single FRB. 


\subsubsection{Neutron Star-Black Hole Mergers}

Mingarelli et al. (2015) suggested that during the inspiral of a neutron starblack hole (NS-BH) merger, the magnetic field lines of the neutron star may thread around the black hole event horizon in a way similar to a battery powering a circuit, generating an electromagnetic pulse which would be observable as an FRB. Black holes interacting with their surrounding environment have also been proposed.

\subsubsection{Black Hole-Black Hole Mergers}

From the abundance of black hole-black hole mergers in the universe, Zhang (2016) theorized that a black hole-black hole merger in which at least one of the spinning black holes carries a certain amount of charge could result in the inspiral process that generates a loop circuit that would induce a global magnetic dipole normal to the orbital plane. During inspiral, as the orbital separation decreases, the magnetic flux of the system changes rapidly, which leads to a magnetospheric outflow with an increasing power and the emission of coherent curvature radiation. Liu et al. (2016) proposed that for merging black hole binaries in which at least one of the black holes is Kerr-Newman (meaning that it is charged and rotating), instabilities due to tidal forces induce reconnection in the Kerr-Newman black holes (KNBH) prior to coalescence. The magnetic field violently reconnects, triggering strong relativistic shock waves through the surrounding plasma to produce curvature radiation consistent with an FRB. They also argued that the merger of a KNBH binary is a plausible central engine for the potential gamma-ray or radio afterglow following 


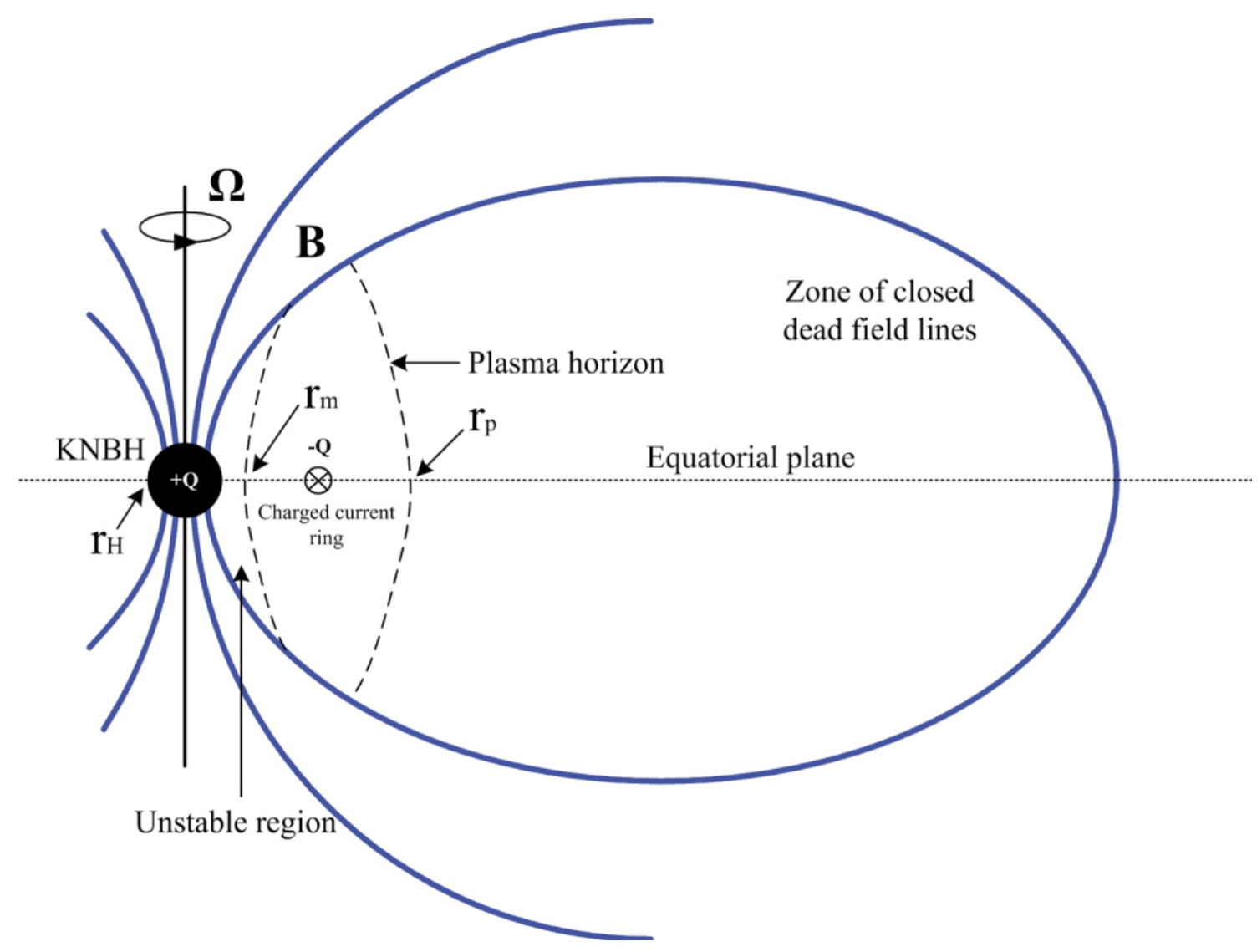

Figure 2.4 Schematic representation of a KNBH. Taken from Liu et al. (2016).

certain FRBs and can also account for gravitational wave events (see Fig. 2.4 for the graphic illustration of a KNBH's initial state).

\subsubsection{Jet-Caviton Models}

Vieyro et al. (2017) proposed a model in which the bursts happen when a non-relativistic electron-positron beam interacts with a cloud at the center of a starforming dwarf galaxy, generating regions of high electrostatic field called "cavitons" in the plasma cloud. Abramowicz et al. (2018) argued that a dark matter halo primordial black hole falls into a Galactic neutron star, rests at the center and accretes the dense matter converting the neutron star into a small $\left(\simeq 1.5 M_{\odot}\right)$ 
black hole and an FRB is formed in the magnetic reconnection event. A complete model of coherent emission in beam-excited plasma cavitons in FRBs is required to explain their energetics and high brightness temperatures (see $§ 1.1 .8)$.

\subsection{Exotic Models}

\subsubsection{Extraterrestrial Intelligence}

Luan \& Goldreich (2014) for the first time considered the high brightness temperatures of FRBs and assessed the possibility that FRBs are signals beamed at Earth by advanced civilizations. Later, Lingam \& Loeb (2017) examined the possibility that beams and optimal frequencies used for powering large light sails could yield parameters that are consistent with FRBs and found they might be artificial in origin. Setting better constraints on FRB observables in future will be helpful for testing the extraterrestrial intelligence models by examining whether some of the FRB constraints are consistent with the constraints for the beams that

may be used to power light sails. Moreover, studying the expected shape of the pulse from a natural burst may allow us to be able to distinguish between FRBs and artificial origins since the diffraction pattern of a beam from a sail would be different based on its shape and have a unique light curve. 


\subsubsection{White Holes}

White hole ${ }^{8}$ progenitors are one of the exotic models suggested so far. If the energy released from a collapsing star reaches the Planck density ${ }^{9}$ it will cease to collapse further and in effect rebound and form a white hole. This is consistent with FRB energetics which suggests a connection between black hole explosions and short radio signals. Barrau et al. (2014) estimated the size of a primordial black hole exploding via a white hole quantum transition. They estimated the power in the resulting explosion and argued that the radio burst caused from this explosion can be accompanied by gamma-ray signals.

\subsubsection{Cosmic Strings}

Another exotic FRB model is related to cosmic strings and although the presence of the localized FRBs with associated galaxy hosts argues against models related to cosmic strings, the present FRB observations cannot completely rule them out. The existence of cosmic strings were initially suggested by Nielsen \& Olesen (1973). They described hypothetical one-dimensional vortex-lines structures moving around as a possible solution for field theories. Kibble (1980) suggested that cosmic strings are topological defects that may have been generated during a symmetry breaking phase transition in the very early universe. Right after the announcement of the Lorimer burst, Vachaspati (2008) proposed that FRBs could be coming from superconducting cosmic strings. After that, many scenarios have been considered in

\footnotetext{
${ }^{8} \mathrm{~A}$ hypothetical highly unstable region of space-time that is essentially the mathematical reverse of a black hole. Unlike black holes, light and matter can only exit a white hole and not enter it.

${ }^{9}$ This is the Planck mass divided by the Planck volume and is equal to $10^{93} \mathrm{~kg} \mathrm{~m}^{-3}$.
} 
which cosmic strings may produce an FRB. For instance, by applying observational factors such as scattering and relativistic effects, Ye et al. (2017) calculated the event rate of three kinds of superconducting cosmic string structures. In addition to directly confronting these predicted rates with observational results, one way of testing this model is through measuring the polarization information of the detected FRBs which are predicted to be linearly polarized at least for one kind of the superconducting cosmic strings. The existence of circularly polarized emission in some FRBs (for a review, see e.g., Cordes \& Chatterjee, 2019) therefore poses problems for superconducting strings as a unifying scheme to explain the entire population.

\subsection{Closing remarks}

In this Chapter we have attempted to survey the already vast body of extant literature devoted to the emission mechanism(s) and source(s) of FRBs. The exotic models discussed in the previous section are energetically viable but invoke sources which are highly speculative in nature. We should keep an open mind for the possibility of multiple source models being possible to explain the FRB phenomenon. Occam's razor points towards the family of models involving compact stellar phenomena (e.g. giant pulses from neutron stars or SLSNe). Much like our understanding of pulsar radio emission, it may take many years to develop a fully self-consistent and physically viable model for FRBs. One of the primary goals of this thesis is to carry out surveys for FRBs which can help further inform and discriminate against the models introduced in this Chapter. 


\section{Chapter 3}

\section{Surveys for FRBs}

Because of the large number of unanswered fundamental questions regarding FRBs, a number of surveys designed to increase the size of the sample have been carried out. In this Chapter, we review the main surveys currently completed and in progress, and summarize the essential techniques used to find them. Table 3.1 summarizes the parameters and results from the current surveys.

\subsection{Single-Dish Surveys}

ALFABURST uses the Arecibo L-band Feed Array (ALFA) to search for FRBs commensally along with other projects (Foster et al., 2018; Chennamangalam et al., 2017). The Pulsar Arecibo L-band Feed Array (PALFA) survey, which is a long-term pulsar survey targeting low Galactic latitudes using the Arecibo L-band Feed Array (ALFA; Cordes et al., 2006), developed a new single-pulse pipeline to efficiently identify single radio pulses from pulsars, rotating radio transients (RRATs), and FRBs and reported on FRB 121102 (Spitler et al., 2014) and candidate FRB 141113 (Patel et al., 2018). The AO327 drift survey is an ongoing FRB search running since 2010 during Arecibo telescope downtime or unassigned time. It aims to search the entire Arecibo sky at $327 \mathrm{MHz}$ (Deneva et al., 2016).

The High Time Resolution Universe (HTRU) high-latitude surveys used the 
Table 3.1 Summary of the Current FRB surveys. The columns correspond to the survey names, the observing telescope, the number of detected FRBs at the time of writing, the observing frequency, the survey sky coverage, the survey observing time, the flux density threshold of the survey for a $5 \mathrm{~ms}$ pulse and $\mathrm{S} / \mathrm{N}$ threshold of 10 , and the reference paper(s).

\begin{tabular}{|c|c|c|c|c|c|c|c|}
\hline Survey & Telescope & FRBs & $\begin{array}{l}\text { Freq. } \\
(\mathrm{MHz})\end{array}$ & $\begin{array}{r}\text { Beam Size } \\
(\operatorname{arcmin})\end{array}$ & $\begin{array}{l}\text { Time } \\
\text { (hr) }\end{array}$ & $\begin{array}{l}S_{\min } \\
(\mathrm{Jy})\end{array}$ & Reference \\
\hline ALFABURST & Arecibo & 0 & 1400 & 3.35 & 518 & 0.04 & Foster et al. (2018) \\
\hline PALFA & Arecibo & 1 & 1375 & 3 & 576 & 0.16 & Spitler et al. (2014) \\
\hline AO327 drift & Arecibo & 0 & 327 & 15 & 882 & 0.20 & Deneva et al. (2016) \\
\hline HTRU & Parkes & 5 & 1352 & 7.5 & 3483 & 0.22 & Champion et al. (2016) \\
\hline SUPERB & Parkes & 4 & 1400 & 7.5 & 2722 & 0.22 & Keane et al. (2018), Bhandari et al. (2018) \\
\hline Archival data & Parkes & 0 & 1374 & 7.5 & 475 & 0.22 & Rane et al. (2016) \\
\hline CHIME/FRB & CHIME & 21 & 600 & 820 & - & 0.30 & CHIME/FRB Collaboration et al. (2019b) \\
\hline CRAFT & ASKAP & 28 & 1296 & 0.12 & - & 7.04 & Macquart et al. (2010) \\
\hline UTMOST & Molonglo & 9 & 843 & 200 & 467 & 4.92 & Caleb et al. (2017) \\
\hline MeerTRAP & MeerKAT & 0 & 1284 & 1 & - & 2.11 & Stappers (2016) \\
\hline LPPS & LOFAR & 0 & 142 & 220 & - & 62 & Coenen et al. (2014) \\
\hline ARTEMIS & LOFAR & 0 & 145 & 220 & 1446 & 62 & Karastergiou et al. (2015) \\
\hline ALERT & LOFAR & 0 & 1400 & 15 & - & 0.46 & Maan \& van Leeuwen (2017) \\
\hline V-FASTR & VLBA & 0 & 1550 & 18 & 4400 & 1.34 & Wayth et al. (2011) \\
\hline MWA pilot & MWA & 0 & 155 & 4.5 & 10.5 & 114 & Tingay et al. (2015) \\
\hline realfast & VLA & 0 & 1396 & 0.75 & 1500 & 0.31 & Law et al. (2018) \\
\hline GBNCC & GBT & 0 & 350 & 35 & 2016 & 0.25 & Chawla et al. (2017) \\
\hline GREENBURST & GBT & 0 & 1400 & 8.8 & 3970 & 0.06 & Surnis et al. (2019) \\
\hline GBTrans & $20-\mathrm{m}$ & 0 & 1400 & 48 & 12072 & 5.20 & Golpayegani et al. (2019) \\
\hline Fly's Eye & ATA & 0 & 1430 & 150 & 450 & 55 & Siemion et al. (2012) \\
\hline
\end{tabular}

Parkes 64-m radio telescope and the Effelsberg 100-m radio telescope (Champion et al., 2016) to cover the sky in three regions for different Galactic latitude ranges.

SUPERB (SUrvey for Pulsars and Extragalactic Radio Bursts) is an ongoing realtime fast transient and pulsar survey at Parkes (Keane et al., 2018; Bhandari et al., 2018) that conducts extensive and rapid multi-messenger post-burst follow-ups at radio, optical, X-ray, neutrino, and gamma-ray facilities. Rane et al. (2016) reported a radio transient and FRB search in Parkes archival data sets. Burke-Spolaor \& Bannister (2014) reported the detection of an FRB in archival intermediate-latitude Parkes Radio Telescope data.

The Green Bank Northern Celestial Cap (GBNCC) Pulsar Survey (Stovall et al., 2014) and GREENBURST (Surnis et al., 2019; Agarwal et al., 2020) are the two main FRB surveys with the Green Bank Telescope. The GBNCC survey started in 2009 with the goal of searching for pulsars and RRATs (Rotating Radio 
Transients). This survey focuses on $350 \mathrm{MHz}$, which can provide strong constraints on the FRB rate and spectral index due to its low frequency range (Chawla et al., 2017). GREENBURST is searching commensally for FRBs at a central frequency of $1.5 \mathrm{GHz}$ with a bandwidth of $800 \mathrm{GHz}$. It is designed to use a parallel tap to the L-band receiver in order to be able to search for FRBs even if other receivers are in focus. GBTrans is another real-time FRB search system using 20-m radio telescope at the Green Bank Observatory and is discussed in detail in Chapter 2.

\subsection{Interferometric Surveys}

CHIME operates in the $400-800 \mathrm{MHz}$ band, and also has a large field of view as well as good sensitivity, which makes this instrument unique for real-time FRB search purposes (The CHIME/FRB Collaboration et al., 2018). Rajwade \& Lorimer (2017) predicted that CHIME will be able to observe $\sim 30$ or more FRBs per day, which is the highest predicted event rate among current FRB surveys. It appears that this prediction is confirmed through the first months of CHIME operation since CHIME discovered nine repeating FRBs and twelve non-repeaters at the time of writing (CHIME/FRB Collaboration et al., 2019a,b; The CHIME/FRB Collaboration et al., 2019).

The Commensal Real-time ASKAP Fast Transients (CRAFT) survey uses the Australian Square Kilometer Array Pathfinder (ASKAP) dishes to search for fast transients (Macquart et al., 2010). CRAFT provides a large sky coverage, but is only sensitive enough to detect bright FRBs ${ }^{1}$.

\footnotetext{
${ }^{1}$ Typically, for small single dishes, there is a trade-off of low sensitivity for large sky coverage.
} 
The Swinburne University of Technology's digital back-end for the Molonglo Observatory Synthesis Telescope array (UTMOST), with the telescope's large collecting area as well as its wide instantaneous field of view, searches for FRBs at $843 \mathrm{MHz}$. As an interferometer, it is capable of localizing FRBs (Caleb et al., 2016). Caleb et al. (2017) reported the first interferometric detections of FRBs at UTMOST. Later, Farah et al. (2018) reported on a new FRB (FRB 170827), detected in real-time at UTMOST, with three temporal components. Most recently UTMOST detected five new FRBs in real-time (Farah et al., 2019).

The LOFAR Pilot Pulsar Survey (LPPS), conducted around $140 \mathrm{MHz}$ (Coenen et al., 2014), ARTEMIS (Advanced Radio Transient Event Monitor and Identification System), a real-time search backend at $145 \mathrm{MHz}$ (Karastergiou et al., 2015), and ALERT (The Apertif LOFAR Exploration of the Radio Transient Sky), a realtime search with Apertif phased array system (Maan \& van Leeuwen, 2017), are three FRB surveys using LOFAR (The Low-Frequency Array).

The MeerTRAP (Meer: more, TRAnsients and Pulsars) project, a real-time commensal pulsar and FRB search using the MeerKAT telescope (Stappers, 2016), benefits from the excellent sensitivity and sky coverage of MeerKAT, which could result in detecting hundreds of well-localized FRBs and their associated hosts. VFASTR (VLBA Fast Radio Transient) commensal experiment, used the Very Long Baseline Array (VLBA) in Socorro, New Mexico in order to search for FRBs (Wayth et al., 2011; Burke-Spolaor et al., 2016), has reported a non-detection on observations up to $100 \mathrm{GHz}$. Tingay et al. (2015) did a pilot study for FRBs using the Murchison Widefield Array (MWA) in Australia at low frequencies (139-170 MHz). Law et al. 
(2015) attempted the first millisecond timescale radio interferometric FRB search at L-Band the VLA. Finally, Realfast is real-time, commensal fast transient survey with the VLA for imaging and FRB detection (Law et al., 2018). Allen Telescope Array (ATA) 'Fly's Eye' survey (Siemion et al., 2012) also took advantage of the large sky coverage of a telescope array with small dishes, jointly operated by the University of California, Berkeley Radio Astronomy Lab and the SETI Institute in Mountain View, California, for 450 hours to search for FRBs at L-band.

\subsection{Single-Dish Search Techniques for FRBs}

FRBs were initially discovered in pulsar survey data collected with single radio dishes (Lorimer et al., 2007). Although radio interferometers are being increasingly employed to search for FRBs due to their superior localization power, the experiments described in this thesis use single dishes. In this section, therefore, I will focus on single-dish techniques.

\subsubsection{Interference Excision}

Radio-frequency interference (RFI) affects radio data in a similar way as light pollution impacts the optical astronomical data. Some of the main terrestrial sources of RFI are cell phones, satellites, radar, Wi-Fi, radio and TV, microwave ovens, and any other devices that work within the observing frequency bandwidth of a radio telescope. When RFI appears it often mimics the features in a burst of astrophysical origin, therefore creating false-alarm signals which can hamper the detection of 
FRBs. Also, RFI decreases the instrument's sensitivity in general. As a result, RFI zapping from the sampled data in frequency and time is the first step toward searching for radio transients.

One simple step is removing all candidates with $\mathrm{DM} \approx 0$, since the free electron density is negligible for terrestrial sources. A powerful technique that is frequently used to eliminate such sources is known as zero dispersion measure filtering (Eatough et al., 2009). In this approach, the mean of the zero dispersion measure time series is subtracted from each frequency channel on a sample-by-sample basis. The resulting frequency channels are then de-dispersed (see below) and searched for non-zero DM signals. By definition, all features with $\mathrm{DM}=0$, and values close to this, are removed from any subsequent analysis. Another technique that is becoming commonly used is to excise signals based on their spectral properties. Following Nita \& Gary (2010), for a set of $N$ samples, we can define the spectral kurtosis

$$
S K=\frac{N+1}{N-1}\left(M \frac{S_{2}}{S_{1}^{2}}-1\right),
$$

where $S_{1}$ and $S_{2}$ are, respectively, the sums of the squares and fourth powers of the set of samples. From the statistics of $S K$, it is possible to automatically identify portions of the data that can be replaced by the equivalent gaussian noise. While both of these techniques necessarily remove parts of the data, the extent of this is usually small $(<1 \%)$. Furthermore, in cases where multi-beam data are available, candidates that appear in all, or almost all, the beams can normally be safely flagged as RFI and removed because such events could only be present if they have entered 
the receiver system indirectly (Petroff et al., 2015).

\subsubsection{De-dispersion}

Radio telescopes are equipped with instrumentation that allows us to record electromagnetic waves as streams of digitized voltages. These data can be stored in a number of different ways using techniques developed primarily to be used to study radio pulsars (Lorimer \& Kramer, 2004). The most common approach, and the one relevant for the majority of FRB searches, is to channelize and square the voltages using a spectrometer. The resulting signals represent intensities as a function of sky frequency and time. These data products are typically referred to as "filterbank" files. Our task when searching for FRBs is to detect and characterizes pulses of unknown width and DM that are hidden in these filterbank files.

Because the DM of an FRB is initially unknown, it is necessary to produce time series from the filterbank files that are optimized for a large range of trial DMs. To remove the $1 / \nu^{2}$ signature present for any astrophysical pulse, for each trial DM, the set of time delays is computed using Eq. 1.1. The time delay of every frequency channel therefore will be calculated for each DM trial and by subtracting the delay for each frequency channel, the quadratic dispersive sweep across the bandwidth will be removed, the data will be summed up in each time sample to produce a "dedispersed time series" corresponding to that DM. Care should be taken to choose the optimal number and step size for the trial DMs used in this process: too many DMs that are finely spaced will result in a waste of computational resources, while too few 
DMs that are coursely spaced will result in a loss of signal-to-noise ratio $(\mathrm{S} / \mathrm{N})$ due to the true DM being significantly away from two neighboring trial values. Fig. 3.1 shows the dynamic spectrum and its integrated pulse profile after being de-dispersed at the optimal DM value of FRB 010312.

\subsubsection{Single-pulse search}

The mean signal level in a time series is called the baseline. The baseline is not always uniform over time and it might fluctuate or have outliers caused by RFI or instrumentational effects. This can result in a non-uniform baseline in the time series, making it difficult to extract astrophysical pulses from the noise. Once de-dispersion is done, the baseline will be estimated by calculating the median or mean of the time series and the outliers above a specified threshold will be removed from the time series, and then the median will be recalculated (Barsdell, 2012). This baseline estimation process is sometimes also known as 'smoothing' (Cordes \& Chatterjee, 2019) and is labelled as such in Fig. 3.2. The 'normalization' step shown below the smoothing refers to the method of computing the root mean square of the smoothed time series so that $\mathrm{S} / \mathrm{N}$ values can readily calculated.

A common technique used to detect individual pulses is to convolve the time series with box-car functions of variable width. This 'matched filter' approach returns the optimal width for each candidate. The resulting $\mathrm{S} / \mathrm{N}$ values from the convolutions are stored for subsequent evaluation as a candidate. In the surveys that we present, the number of trial widths is 12 (Chapter 4) and 16 (Chapter 5). 


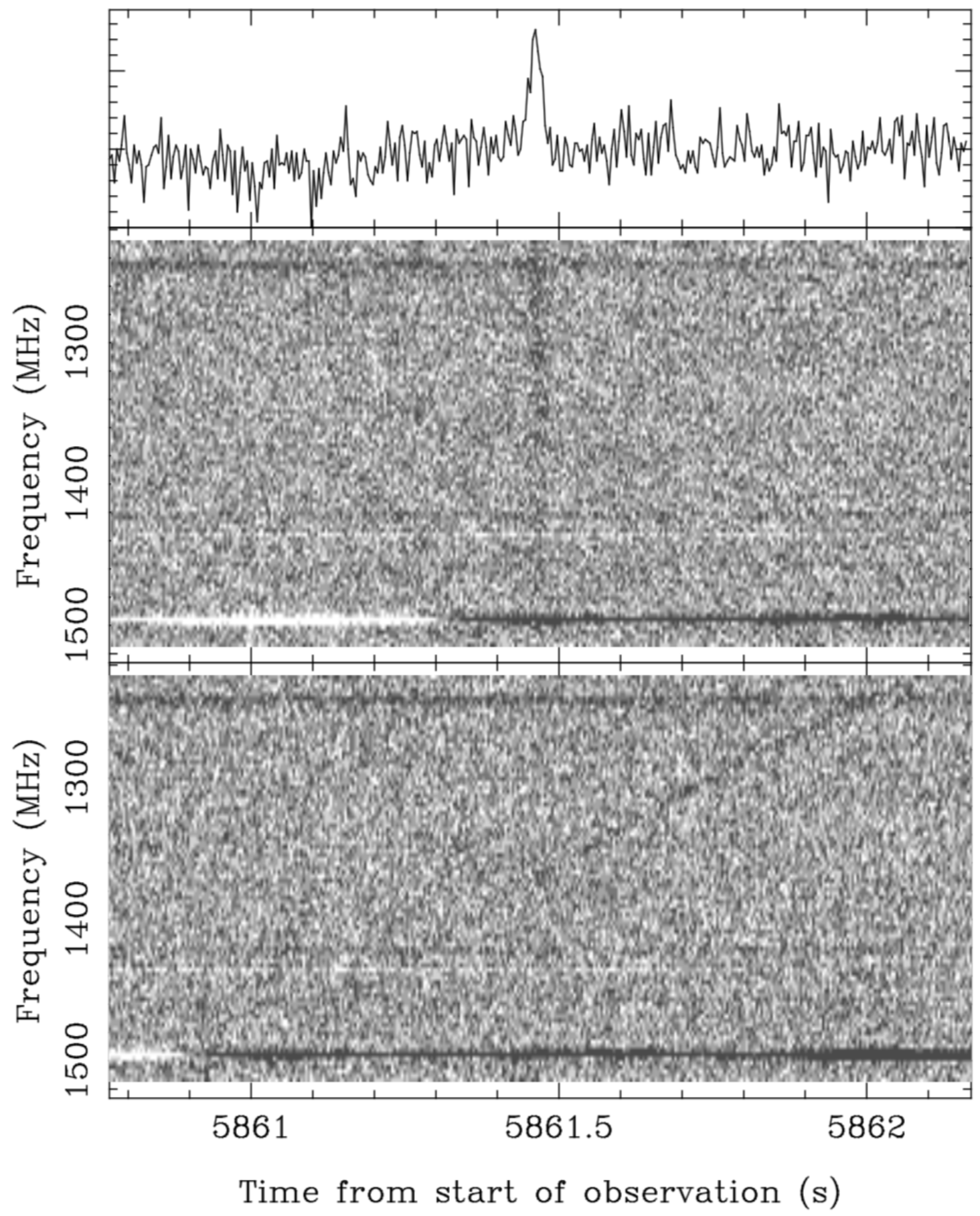

Figure 3.1 Dynamic spectrum of the FRB 010312 without dispersion (bottom), with dispersion at the optimal DM (central), and its integrated pulse profile with an arbitrary flux units) after being de-dispersed at the optimal DM value (top). The frequency resolution is $3 \mathrm{MHz}$ and the time axis shows the MJD at the beginning of the observation with the time resolutuon of $4 \mathrm{~ms}$. It is noticeable that the signal is much stronger in lower frequencies. Taken from Zhang et al. (2019). 
At this stage, no discrimination is made on DM or pulse widths from all of the candidates coming out of the matched filtering analysis. Grouping the candidates in clusters, where each cluster corresponds to a specific event with an optimal DM and pulse width, can be done using the "friends-of-friends" algorithm (Huchra \& Geller, 1982). Using this algorithm, events with the same DM within a defined range $\left(\approx 20 \mathrm{~cm}^{-3} \mathrm{pc}\right)$, a defined range of time of arrival, can be grouped and then a single cluster with an event of the highest $\mathrm{S} / \mathrm{N}$ will be saved. Fig. 3.2 demonstrates a summary of the single-pulse search procedure in a block diagram.

\subsubsection{Candidate selection}

Once the event windows were selected according to some criteria as part of a single-pulse search, a post-processing search algorithm is typically used for candidate classification. This is necessary because the searches are affected by a high false positive rate caused by RFI and noise fluctuations. While early searches made use of manual classification of candidates (see, e.g., Lorimer et al., 2007), the large number of events quickly demanded more sophisticated and automated techniques. While significant progress has been made with the implementation of automated scoring algorithms (see, e.g. Karako-Argaman et al., 2015), machine learning algorithms are becoming increasingly popular ways to address the problem. For instance, Devine et al. (2016) developed a method for identifying and classifying clustered groups of dispersed pulses in single-pulse search output. There, 16 group features (e.g., startend DM, maximum $\mathrm{S} / \mathrm{N}$ ) are used in six supervised algorithms to find the 


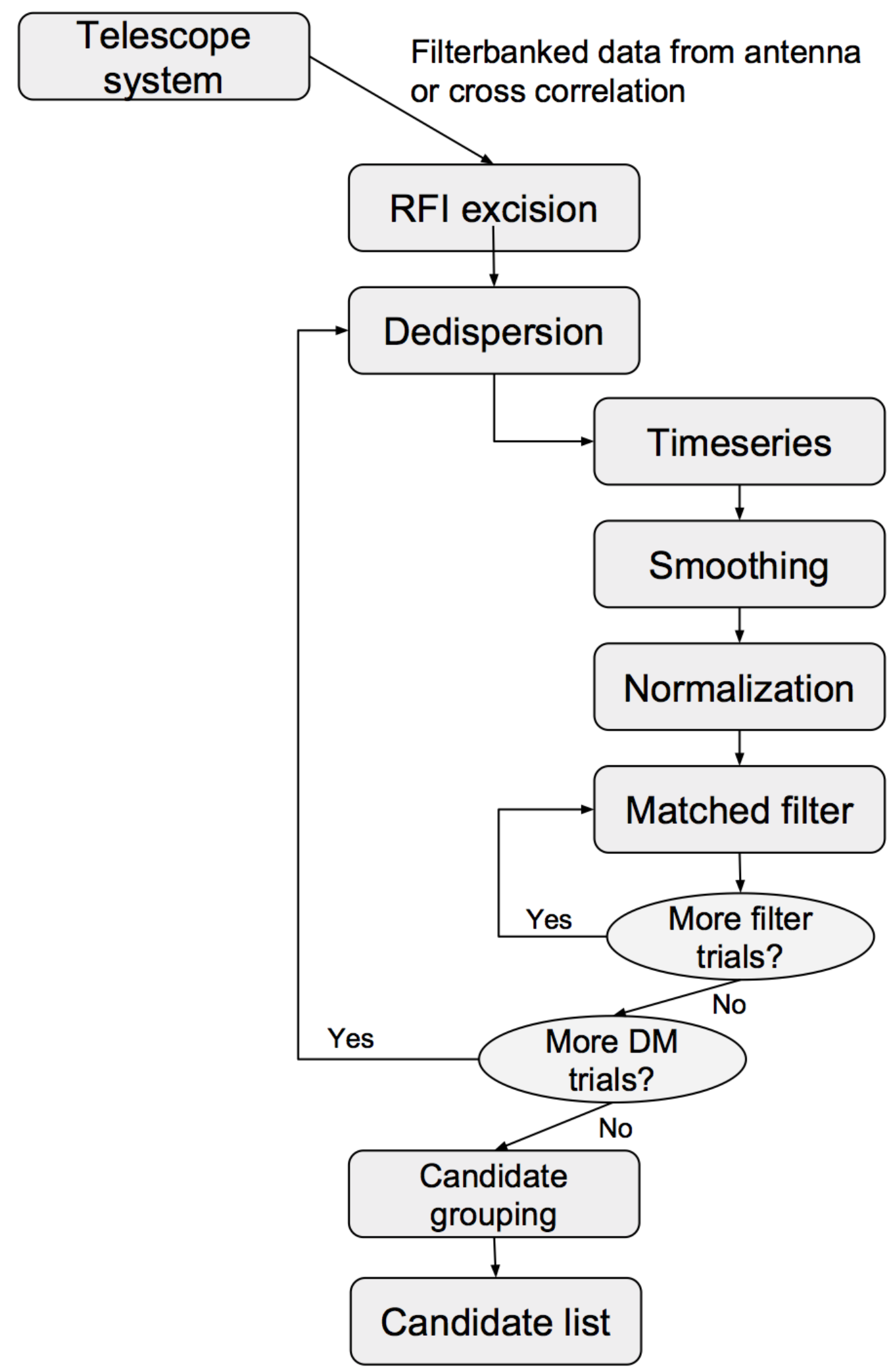

Figure 3.2 Block diagram summarizing the single-pulse search procedure. Taken from Cordes \& Chatterjee (2019). 
best combination of hyperparameters and classifier. The speed and accuracy of this method later was improved by Pang et al. (2018). Connor \& van Leeuwen (2018) applied a deep neural network to the single-pulse classification problem that takes data products such as dynamic spectra as input and trains on them simultaneously by using false-positive triggers from real telescopes, simulated FRBs, and pulsar single pulses as training sets.

One recent technique that has been designed to classify FRB candidates in real time is known as FETCH (Fast Extragalactic Transient Candidate Hunter; Agarwal et al., 2019). FETCH is designed to use the technique of transfer learning to work on frequency-time as well as DM-time images as the input images to distinguish FRB candidates from RFIs by training the model. The input images include simulated FRBs, real RFI candidates, and real pulses from known pulsars. The model is trained on the L-band data from the Green Bank Telescope as well as 20-m telescope described in Chapter 4. The resulting algorithm is independent from the telescope and the observing frequency, making it an excellent post-processing model for various commensal FRB search pipelines for real-time candidate classification. Fig. 3.3 shows sample images from the training and test dataset, which includes the time series for visual purposes, the de-dispersed frequency-time spectrograms of a fixed size $(32 \times 64$ pixels $)$, and the DM-time grids reflecting the $\mathrm{S} / \mathrm{N}$ of the integrated pulse profile $(100 \times 64$ pixels $)$. 

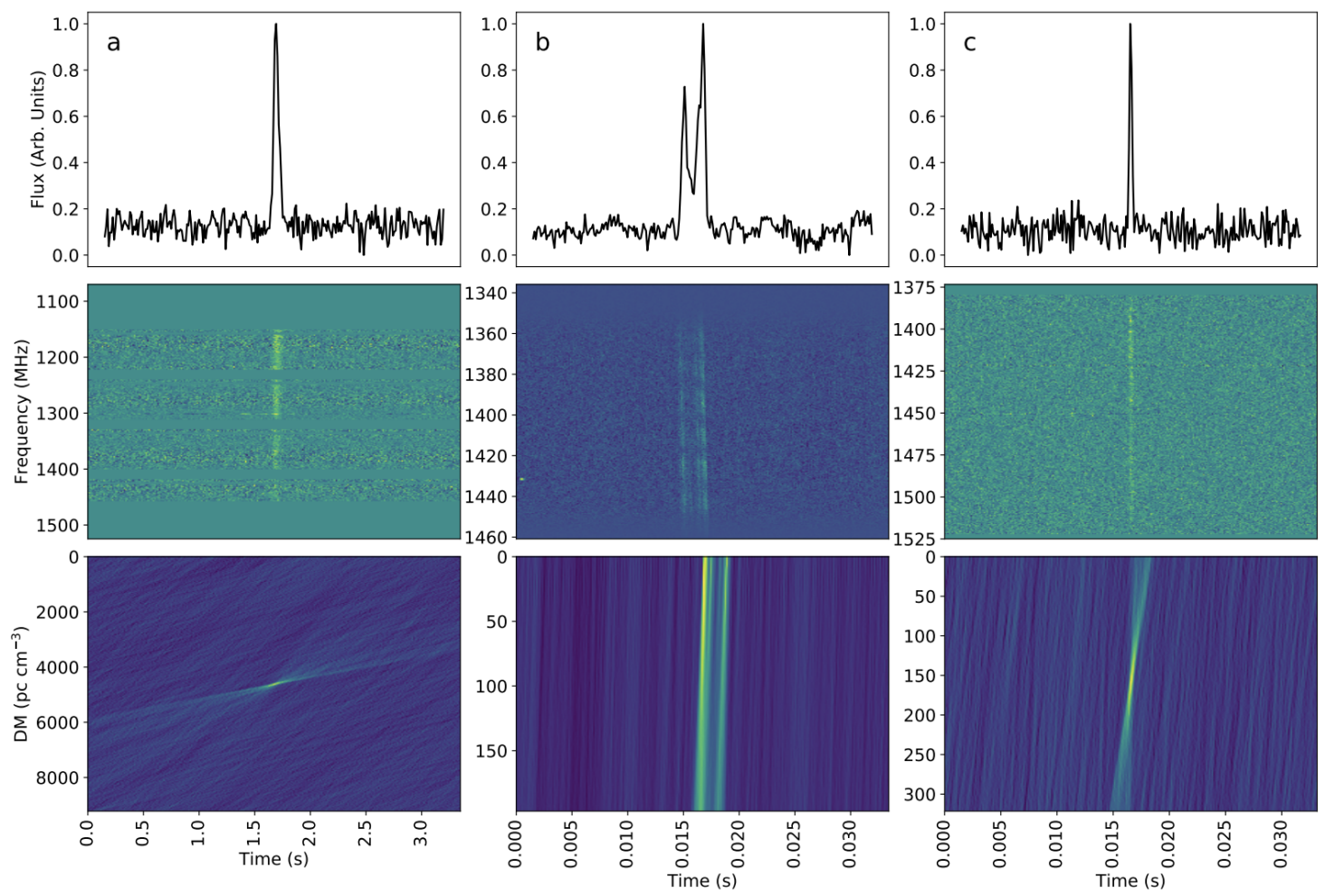

Figure 3.3 Sample FRB candidate images from the training and test datasets used by FETCH. Top: time-series profile, which is included for visual reference. Middle: frequency-time image. Bottom: DM-time image. Column (a) corresponds to a simulated FRB superposed into a background data from FLAG (the Focal L-Band Array for the Green Bank Telescope). Column (b) is a real RFI candidate from the Green Bank 20-m telescope. Column (c) is a real pulsar observed using the FLAG system. Taken from Agarwal et al. (2019). 


\subsubsection{Sensitivity}

For any radio source, its flux density, $S_{\nu}$, at some specific frequency, $\nu$, can be related (see, e.g., Lorimer \& Kramer, 2004) to the antenna temperature, $T_{A}$, and the effective area of the dish, $A_{\text {eff }}$, as follows:

$$
S_{\nu} A_{\mathrm{eff}}=2 k T_{A}
$$

where $k$ is Boltzmann's constant. From this expression, we infer the ratio of $T_{A} / S_{\text {nu }}$ to be the antenna gain, $G=A /(2 k)$.

Now, for a top-hat pulse of width $W$, and peak flux density $S_{\text {peak }}$, radiometer noise considerations mean that the signal-to-noise ratio

$$
\mathrm{S} / \mathrm{N}=\frac{G S_{\mathrm{peak}}}{T_{\mathrm{sys}}} \sqrt{n_{p} W \Delta \nu}
$$

where $n_{p}$ is the number of polarizations summed (usually two), $\Delta \nu$ is the system bandwdith and the system temperature

$$
T_{\mathrm{sys}}=T_{\mathrm{CMB}}+T_{\text {source }}+T_{\mathrm{atm}}+T_{\text {spill }}+T_{\mathrm{rec}}+T_{\text {sky }} .
$$

In this expression, the relevant contributions are: (i) $T_{\mathrm{CMB}}$, the $2.73 \mathrm{~K}$ black-body temperature of the cosmic microwave background (CMB); (ii) $T_{\text {source, }}$, the source's contribution, which is typically less than $0.01 \mathrm{~K}$ and therefore negligible; (iii) $T_{\text {atm }}$, the temperature from turbulent behavior from the Earth's atmosphere, which is 
negligible if the observing frequency is less than $5 \mathrm{GHz}$; (iv) $T_{\text {spill }}$, is the radiation temperature the feed picks up in directions beyond the edge of the dish's reflector (e.g. the reflections from the ground), which is usually no more than $10 \mathrm{~K}$; (v) $T_{\text {rec}}$, the receiver's contribution and is typically around $20 \mathrm{~K}$; (vi) $T_{\text {sky }}$, the sky background contribution, which is strongly dependent on the observing frequency and the direction of the sky. Typical $T_{\text {sky }}$ values at $1.4 \mathrm{GHz}$ are $\sim 5-20 \mathrm{~K}$ depending on how close the line of sight passes through the Galactic plane.

To conclude this chapter I compute the sensitivity of the HTRU survey as an example using the survey parameters given in Champion et al. (2016). I take the measured gain of the Parkes telescope with $G=0.69 \mathrm{~K} \mathrm{Jy}^{-1}$ and using the radiometer equation compute the minimum detectable flux density

$$
\mathcal{S}_{\min }=\frac{T_{\mathrm{sys}} \mathrm{S} / \mathrm{N}}{G \sqrt{n_{p} W \Delta \nu}},
$$

where the typical system temperature $T_{\text {sys }}=28 \mathrm{~K}$, number of polarization is 2 , and the bandwidth $\Delta \nu=338.281 \mathrm{MHz}$ for the observing system. Adopting the minimum FRB pulse width $W=5 \mathrm{~ms}$, and a $\mathrm{S} / \mathrm{N}$ threshold of 9 gives the minimum detectable flux density $\mathcal{S}_{\min } \simeq 0.2$ Jy for the HTRU survey. 


\section{Chapter 4}

\section{A search for FRBs with the Green Bank 20-m Telescope}

The material presented in this Chapter is based on a paper published by Golpayegani et al. (2019) and has been edited slightly for stylistic purposes.

\subsection{Introduction}

Many of the FRB surveys carried out so far (Petroff et al., 2019, see their section 4.3 for a review) used telescopes with large collecting areas and high sensitivities, motivated by the relatively large flux densities of some FRBs (see, e.g., Shannon et al., 2018), we have developed a real-time FRB detector on the 20-m telescope at the Green Bank Observatory, taking advantage of the extensive sky coverage available (approximately $80 \%$ of the sky) and a large field of view of this smaller dish. This experiment, which we call GBTrans, is a synergistic effort partially supported from the Skynet Robotic Telescope Network Project ${ }^{1}$. The 20-m telescope is also being used in a companion project (Gregg et al. 2019), which focuses on coordinated observations with the Neil Gehrels Swift Observatory. The plan for the rest of this Chapter is as follows. In section 4.2, we describe the GBTrans system and detection pipeline. In section 4.3, we summarize the observations carried out and present the results of the survey including detected single pulses and

\footnotetext{
${ }^{1}$ http://skynet.unc.edu
} 


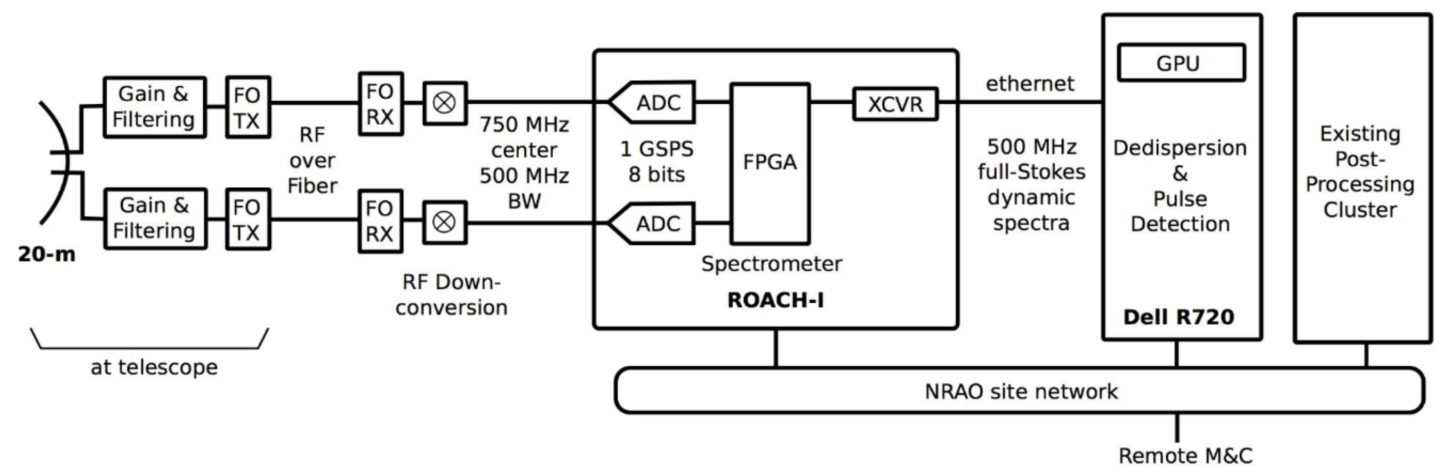

Figure 4.1 Block diagram showing the downstream electronics and data acquisition system summarising the existing system architecture developed for GBTrans.

giant pulses from known pulsars and candidate astrophysical pulses. In section 4.4 we explain the method we used to estimate the FRB rate and survey volume for this survey and possible explanations for our non-detection of FRBs so far and speculate on future developments.

\subsection{GBTrans Description}

The 20-m telescope at the Green Bank Observatory in Green Bank, WV, has been in operation since late 1994. Originally funded by the US Naval Observatory, it was part of the National Earth Orientation Service telescope network, and participated in a global program of Earth Orientation VLBI (very long baseline interferometry) measurements in cooperation with the International Earth Rotation Service, and with the NASA Space Geodesy program. Following a shut down in 2000, the telescope was restored, automated, and made accessible as part of Skynet (Smith et al., 2016; Hosmer et al., 2013). The main receiver currently in use operates at a central frequency of $1.4 \mathrm{GHz}$ and provides a cryogenically cooled 
dual-polarization channel input for pulsar and spectral line work. Although there was some variation due to occasional cryogenics failures as well as telescope time spent on bright sources, the typical system temperature was found to be $40 \mathrm{~K}$. The observing bandwidth is $80 \mathrm{MHz}$, centred at $1400 \mathrm{MHz}$.

A block diagram summarising the signal path from the sky to the data acquisition system developed for GBTrans is shown in Fig. 4.1. The signals are down-converted to a centre frequency of $750 \mathrm{MHz}$ and digitized at $1 \mathrm{GHz}$ before being converted to incoherent fully-polarimetric dynamic spectra using a ROACH-I FPGA-based spectrometer. The spectrometer output is 2048 frequency channels with a spectral resolution of $244 \mathrm{kHz}$ and time resolution of $131 \mu \mathrm{s}$, represented as 8-bit integers for all four Stokes parameters. The resulting data stream is slightly greater than $500 \mathrm{Mb} / \mathrm{s}$, including meta-data.

Real time analysis and detection is implemented on a GPU-equipped Dell R720 rack mount server using purpose-built software developed by Virginia Tech. The server consists of dual Intel Xeon E5-2640 2.5 GHz 6-core CPUs, 32 GB RAM, $4 \times 1$ TB hot-pluggable hard drives, and an Nvidia Tesla K10 Graphics Processing Unit (GPU). Data analysis software is implemented in $\mathrm{C}$ and was developed to run on a Linux platform. The principal software components include a ring buffer, an executive processor, and a GPU-based processor. The ring buffer transfers data arriving synchronously from the spectrometer into shared memory, which allows the executive processor to operate asynchronously. The executive processor operates on arriving dynamic spectra in contiguous 13.1-s segments. Each segment is examined for data integrity (e.g., checking for correctly-ordered frame counters). As a diag- 
nostic, spectra and total power for all four Stokes parameters are integrated over the segment and recorded.

We take the 13.1-s data segments and use a GPU to produce de-dispersed total power time series using a brute-force algorithm for 531 trial dispersion measures (DMs) spanning the range $0-9900 \mathrm{~cm}^{-3}$ pc. Each time series is subsequently box-car averaged in powers of 2 to search for single-sample pulses with widths in the range $131 \mu \mathrm{s}$ to $268 \mathrm{~ms}$. The resulting detection metrics are saved, and any data segments containing pulses with signal-to-noise $(\mathrm{S} / \mathrm{N})$ ratios exceeding 10 and DM $>10$ trigger a data-preservation protocol which causes a block of data to be written which we henceforth refer to as an event. Each event consists of the raw segment of full-Stokes data as well as all available meta-data which is saved on a post-processing cluster for long-term storage and follow-up analysis.

\subsection{Observations and Data Processing}

We have collected data with the aforementioned system from the beginning of December 2014 to the beginning of March 2018. Taking into account the days in which the system was down due to maintenance on the telescope or equipment failures where no events were recorded, GBTrans was in operation for 503 days. Fig. 4.2 shows the distribution of events over the entire duration.

For each event, we applied a post-detection pipeline where the data were processed using the HEIMDALL ${ }^{1}$ single-pulse software package. The generated candidates were clustered using the "friends-of-friends" algorithm (Huchra \& Geller,

\footnotetext{
${ }^{1}$ https://sourceforge.net/projects/heimdall-astro
} 


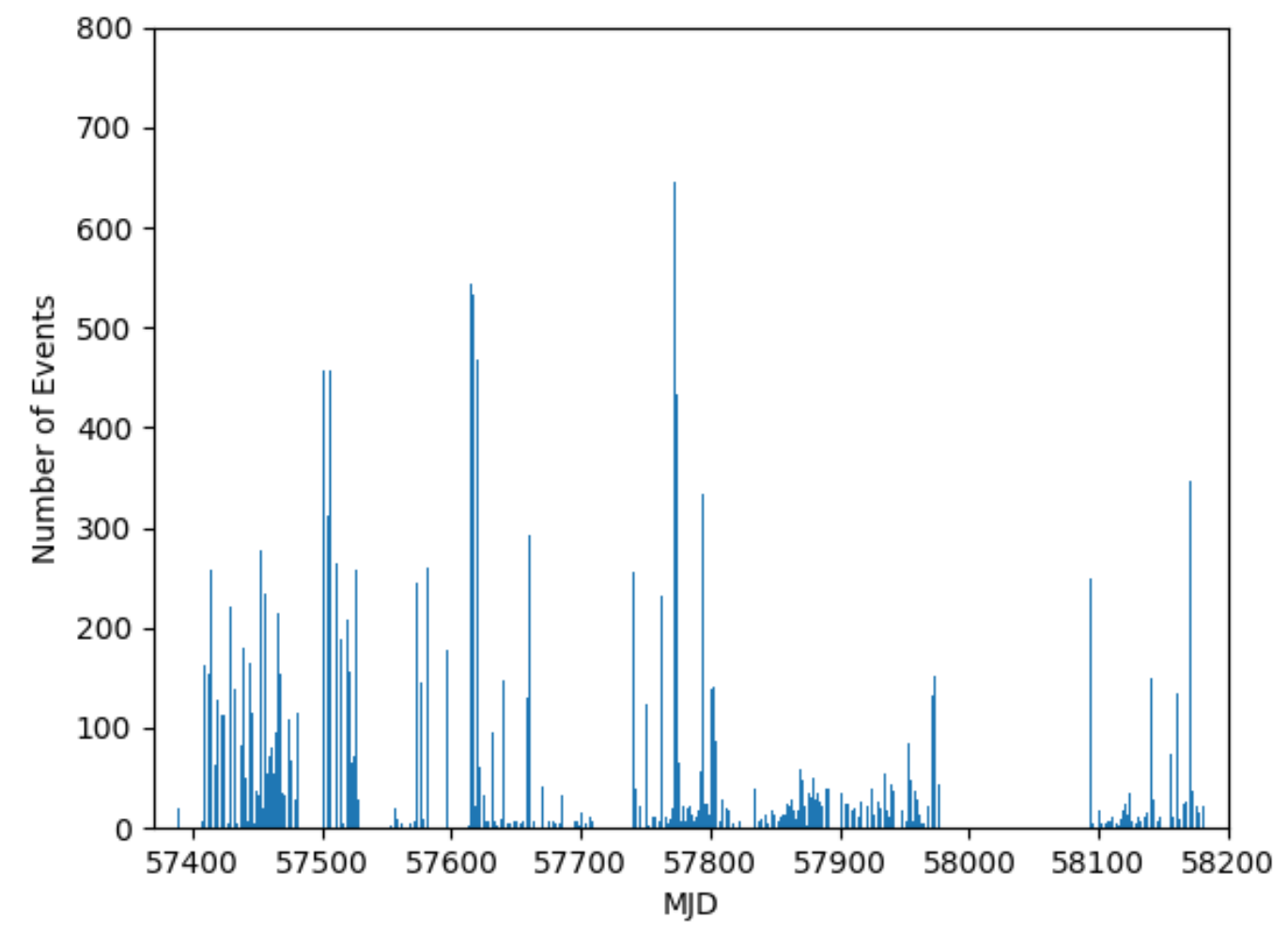

Figure 4.2 Histogram of the number of events versus date. There is very little data available before January 2016 (Seven epochs containing 27 events at MJDs from from 56998 to 57121). Most of our observations occurred between January 2016 and September 2017 (MJD range 57400 - 58000). 
1982) in which groups of events were identified with the same DM within a tolerance of $20 \mathrm{~cm}^{-3} \mathrm{pc}$, a time of arrival within $4.192 \mathrm{~ms}$, and associated with an event of the highest $\mathrm{S} / \mathrm{N}$ and pulse width. The resulting candidates were then appended to the output list and tested against the following criteria: Pulse widths shorter than $33.5 \mathrm{~ms}$ and $\mathrm{S} / \mathrm{N}$ above 10. For each event that met these constraints, a diagnostic plot was generated that contained the original dynamic spectrum, the de-dispersed dynamic spectrum using the DM at which the pulse was detected with the highest $\mathrm{S} / \mathrm{N}$, along with a frequency collapsed time series of the detection with the length equal to twice the DM-dependant delay time, and were inspected visually. These were reviewed and categorised into three classes: known pulsars, radio-frequency interference and unidentified single pulses (i.e. candidate FRBs). Five known pulsars were labelled as such by cross-correlating meta-data from the headers with the ATNF pulsar catalogue (Manchester et al., 2005) ${ }^{2}$. The detection statistics for these pulsars are summarized in Table 4.1. The other two classes were reviewed and labelled manually. Although we did not detect any FRBs in this analysis, we did detect $\sim 22117$ giant pulses from the Crab pulsar which will be published elsewhere as part of a dedicated study of the Crab. An example Crab giant pulse is shown in Fig. 4.3. Since L-band is more problematic in terms of RFI than other observing frequencies, a significant amount of RFI events were present in our candidate output plots. In particular, 25115 false positive events, excluding the single pulses from known sources were detected. Fig. 4.4. shows an exotic RFI candidate example caused by a frequency-modulation continuous wave radar.

\footnotetext{
${ }^{2}$ http://www.atnf.csiro.au/research/pulsar/psrcat
} 

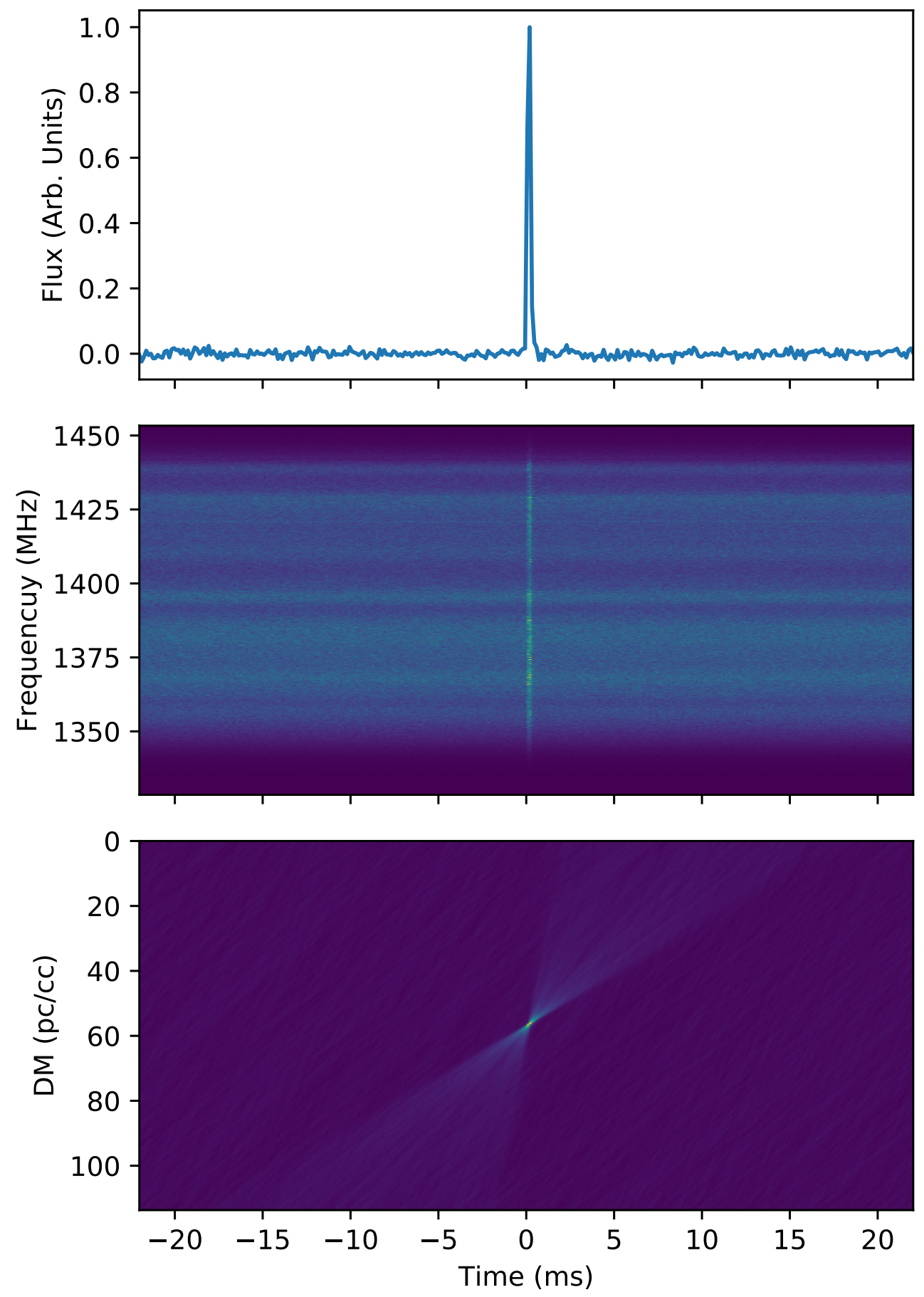

Figure 4.3 An example giant pulse $(\mathrm{S} / \mathrm{N} \sim 90)$ detected from the Crab pulsar, J0534+2200. The top panel shows the de-dispersed pulse in arbitrary flux units normalized to the peak intensity, the middle panel is the de-dispersed pulse in the frequency-time domain, and the bottom panel is the DM-time image. The $40 \mathrm{~ms}$ window is determined by doubling the DM-dependent dispersion delay of the pulse. 

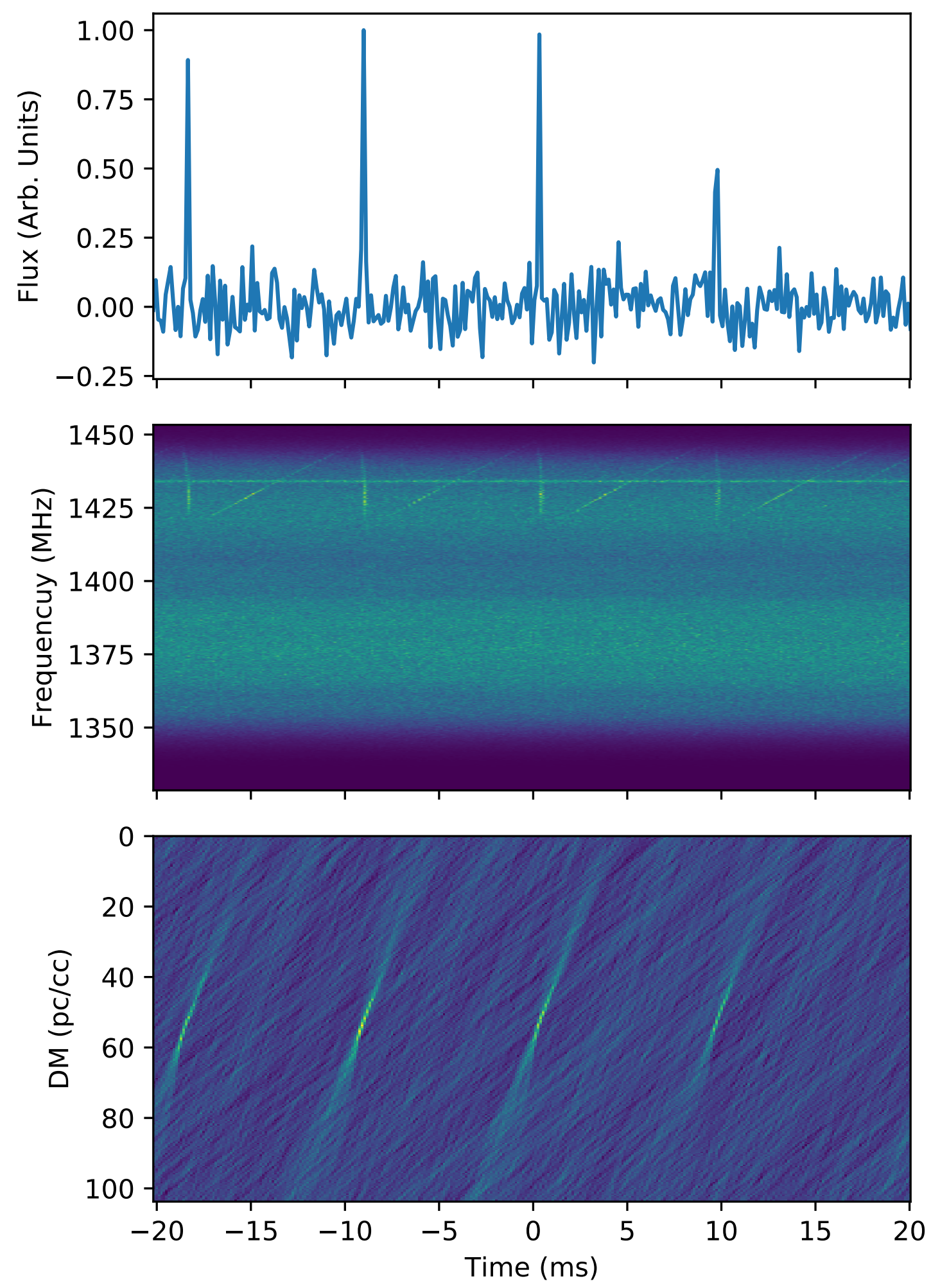

Figure 4.4 Example radio frequency interference signal caused by a frequencymodulation continuous wave radar. The top panel show the individual pulses, the middle panel is the de-dispersed pulses in frequency-time domain, and the bottom panel is the DM-time image. 


\begin{tabular}{cllll}
\hline \multicolumn{1}{c}{ PSR } & $\begin{array}{c}\text { S1400 } \\
(\mathrm{mJy})\end{array}$ & $\begin{array}{c}\text { DMcat } \\
(\mathrm{cm}-3 \mathrm{pc})\end{array}$ & Npulses & S/Nmax \\
\hline J0332+5434 & 203 & 26.76 & 344 & 13.05 \\
$\mathrm{~J} 0534+2200$ & 14 & 56.77 & 22117 & 88.69 \\
$\mathrm{~J} 0835-4510$ & 1050 & 67.97 & 13 & 10.51 \\
$\mathrm{~J} 1644-4459$ & 296 & 478.80 & 318 & 34.38 \\
$\mathrm{~J} 2022+5154$ & 27 & 22.55 & 1633 & 13.09 \\
\hline
\end{tabular}

Table 4.1 Parameters for known pulsars detected by GBTrans. From left to right, we list pulsar name, mean flux density at $1400 \mathrm{MHz}$, catalogue DM, number of detected single-pulses, and maximum single-pulse S/N. The mean flux density at $1400 \mathrm{MHz}$ and DM were obtained from the ATNF pulsar catalogue (Manchester et al., 2005).

\subsection{Discussion}

\subsubsection{Expected FRB Rate}

When this experiment was being designed in 2013, the all-sky FRB rate, $\mathcal{R}$, was thought to be much higher than current estimates, which are now based on larger samples of FRBs. Recent studies (see, e.g., Lawrence et al., 2017) now show that the event rate is almost an order of magnitude lower than previously thought (see, e.g., Thornton et al., 2013). With this in mind, the lack of FRB detections in the survey, while disappointing, still provides useful constraints on the rate-fluence distribution. In our analysis below, we first determine the instantaneous sensitivity and field of view of our experiment to FRBs. We then adopt a recent determination of the all-sky FRB rate $\mathcal{R}_{\mathrm{ASKAP}}=37 \pm 8$ bursts per sky per day with $1.4 \mathrm{GHz}$ fluences above $26 \mathrm{Jy}$ ms, which was found from an analysis of ASKAP detections (Shannon et al., 2018), to determine realistic expectation times needed to make a detection. 
To compute the sensitivity and sky coverage of GBTrans, we take the measured gain of the $20-\mathrm{m}$ telescope, $G=A / 2 k$, where the effective surface area $A=237 \mathrm{~m}^{2}$ assumes an aperture efficiency of $75 \%$ based on the modeled feed patterns, and $k$ is Boltzmann's constant. From this, we find $G=0.086 \mathrm{~K} \mathrm{Jy}^{-1}$. Next, we use the radiometer equation (see, e.g., Lorimer \& Kramer, 2004) to compute for some limiting signal-to-noise ratio $(\mathrm{S} / \mathrm{N})$ the minimum detectable fluence

$$
\mathcal{F}_{\text {min }}=\frac{T_{\text {sys }} \mathrm{S} / \mathrm{N}}{G} \sqrt{\frac{W}{2 \Delta \nu}}
$$

where the typical system temperature $T_{\text {sys }}=40 \mathrm{~K}$ and the bandwidth $B=80 \mathrm{MHz}$. For consistency with the ASKAP survey, we adopt their minimum FRB pulse width $W=1.26 \mathrm{~ms}$, and a $\mathrm{S} / \mathrm{N}$ threshold of 10 . This gives $\mathcal{F}_{\min } \simeq 6 \mathrm{Jy}$ ms. The minimum detectable fluence at the full-width half maximum (FWHM) of the main beam of GBTrans is therefore about $12 \mathrm{Jy} \mathrm{ms}$. We adopt this as a fluence-complete limit in the analysis below. Fig. 4.5 shows the survey sensitivity among with previously detected FRBs. It appears that more than half of the current FRBs are detectable with GBTrans. Because our time resolution is better than for the ASKAP survey, as shown in Fig. 4.5, we also are sensitive to FRBs with widths shorter than $1 \mathrm{~ms}$. Such FRBs do exist, and the observed sample is currently dominated by CHIME discoveries (CHIME/FRB Collaboration et al., 2019b). Because of the scaling between fluence and pulse width in Equation 4.1, the fluence limit at our sampling interval $(131 \mu \mathrm{s})$ is about $4 \mathrm{Jy} \mathrm{ms}$.

Having found the sensitivity out to the beam FWHM, we next need to compute 
the corresponding solid angle, $\Omega$, which represents the instantaneous amount of sky sampled at this limit. For a gaussian beam response (for a discussion, see Condon \& Ransom, 2016) we have $\Omega \simeq 1.133 \mathrm{FWHM}^{2}$, where for an observing wavelength, $\lambda=0.2 \mathrm{~m}$

$$
\mathrm{FWHM}=1.2 \frac{\lambda}{\sqrt{4 A / \pi}}=48^{\prime}
$$

From this, we find that the beam solid angle at the FWHM, $\Omega=2.2 \times 10^{-4}$ sr or $1.7 \times 10^{-5}$ of the whole sky.

We model the rate-fluence distribution as a power law such that

$$
\mathcal{R}(>\mathcal{F})=\mathcal{R}_{\text {ASKAP }}\left(\frac{\mathcal{F}}{26 \text { Jy ms }}\right)^{-\alpha}
$$

where the index $\alpha=1.5$ for Euclidean geometry. Keeping $\alpha$ as a free parameter but setting $\mathcal{F}=\mathcal{F}_{\text {min }}$, then we find an expression for the mean "waiting time", $T$, to detect a pulse. Since this is just the reciprocal of the rate scaled by the solid angle coverage, we find that

$$
T=(\mathcal{R} \Omega)^{-1}=\left(\frac{1600 \pm 350 \text { days }}{2.2^{\alpha}}\right)
$$

In Fig. 4.6 we show Eq. 4.4 alongside these various values of $\alpha$ from earlier studies and our experimental limit on $T$. To be consistent with our experimental results, $T>503$ days. From this, as shown in Fig. 6, we estimate that $\alpha<1.7$.

Care should be taken when interpreting this simple estimate of the upper limit because there is no confidence interval associated with it. To demonstrate this, 


\begin{tabular}{l|l} 
Source count index, $\alpha$ & Reference \\
\hline $2.6_{+1.3}^{-0.7}$ & Macquart \& Ekers (2018) \\
$2.2_{+0.6}^{-1.2}$ & Bhandari et al. (2018) \\
$2.20 \pm 0.47$ (ASKAP) & James et al. (2019) \\
$2.1_{+0.5}^{-0.6}$ & Shannon et al. (2018) \\
$<2.5$ & GBTrans - this Chapter \\
$1.18 \pm 0.24$ (Parkes) & James et al. (2019) \\
0.91 & Lawrence et al. (2017) \\
$0.8-1.7$ & Oppermann et al. (2016) \\
$0.9 \pm 0.3$ & Caleb et al. (2016) \\
$0.5-0.9$ & Vedantham et al. (2016) \\
$0.14 \pm 0.20$ & Li et al. (2017)
\end{tabular}

Table 4.2 Various estimates on $\alpha$ values ranked in descending order. Our constraint of $\alpha<2$ is consistent with a number of estimates, as well as the expectation for a population of standard candles in Euclidean geometry in which $\alpha=1.5$.

assuming that FRBs as a population follow Poissonian statistics in their event rate, the probability of finding at least one FRB in our data set $P_{1}=1-\exp (-\mathcal{R} \Omega T)$. Setting $\alpha=1.7$ in Equation 4.3 to find $\mathcal{R}$ and $T=503$ days, we find $P_{1}=70 \%$. To set a robust limit on $\alpha$, we can repeat this calculation to find $P_{1}$ as a function of $\alpha$. Requiring $P_{1} \geq 0.9$, we find that we should have detected at least one FRB with $90 \%$ confidence if $\alpha>2.5$. We therefore conclude that $\alpha<2.5$ at the $90 \%$ confidence level.

Table 4.2 summarizes different $\alpha$ constraints reported in literature. There is currently a wide range of $\alpha$ values that are quoted. A Euclidean rate-fluence distribution would therefore lead to $T \sim 1$ yr. Macquart \& Ekers (2018) estimate, based on a recent maximum likelihood analysis on the Parkes FRBs, that $\alpha=$ $2.6_{+1.3}^{-0.7}$. For this range of $\alpha$ values, we would expect waiting times in the range $58<T<436$ days. In contrast, Li et al. (2017) estimate $\alpha=0.14 \pm 0.20$. This would correspond to $956<T<2044$ days. 


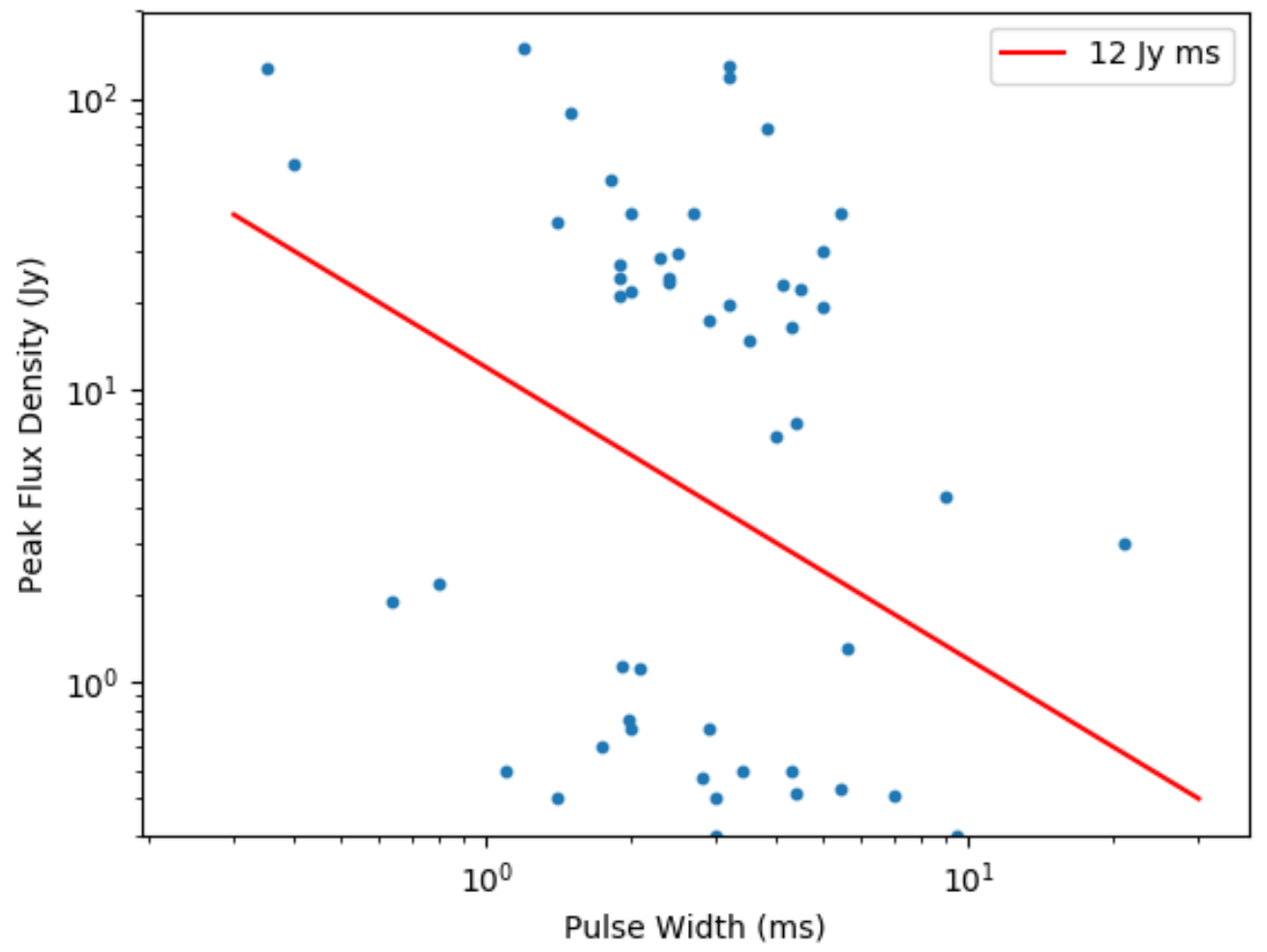

Figure 4.5 Survey sensitivity of GBTrans based pulse width and peak flux density. The red line is the derived minimum detectable fluence. Blue dots are previously reported FRBs and events above this line are detectable with GBTrans. 


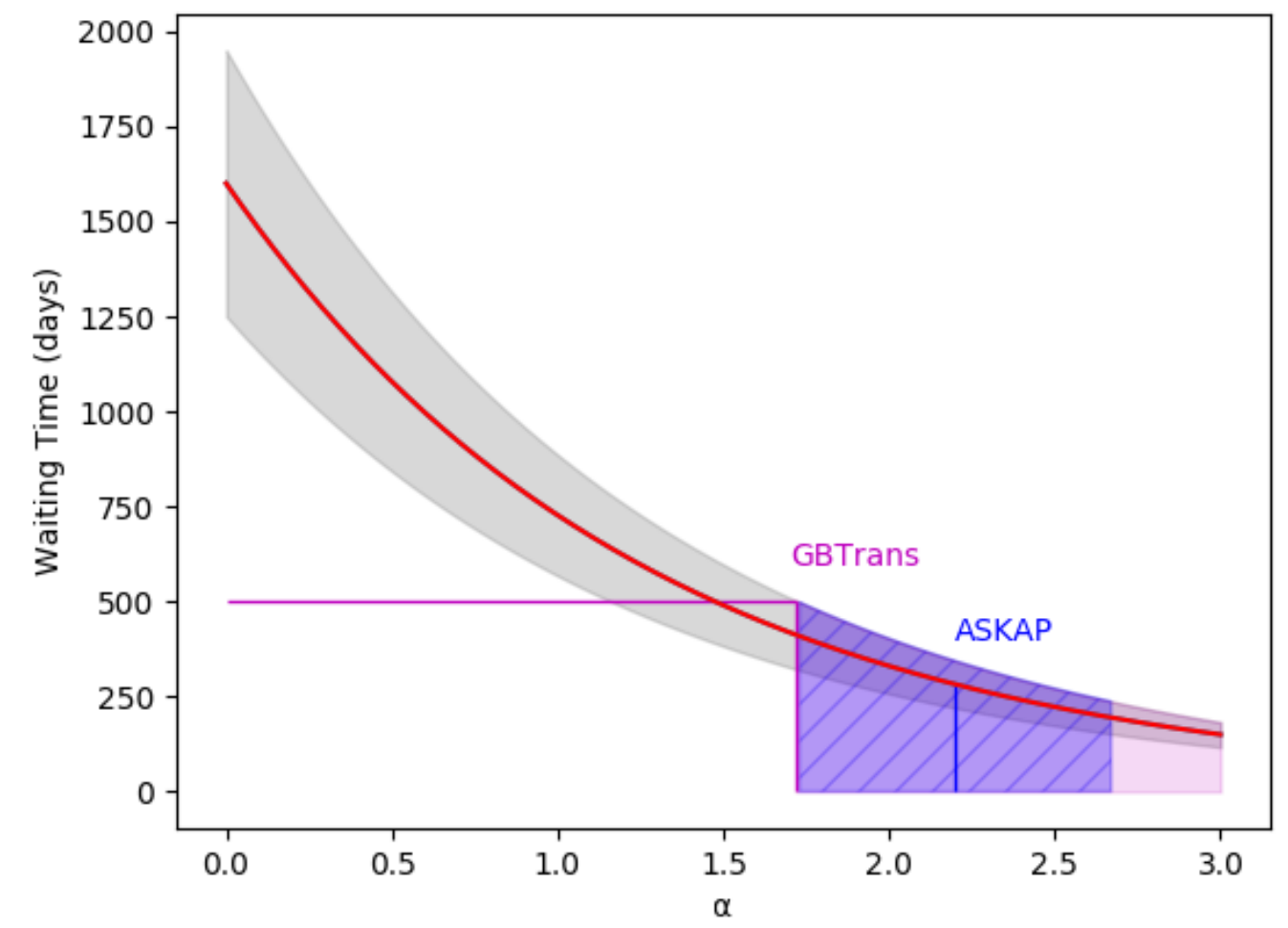

Figure 4.6 Expected waiting time (red line) for GBTrans as a function of sourcecount index $\alpha$. The shaded grey region is the uncertainty of the function. The blue line is the $\alpha$ value estimation from ASKAP with its shaded blue uncertainty region (James et al., 2019). The pink line demonstrates the corresponding $\alpha$ value of this survey considering our non-detection after 503 days of observation. The shaded pink region is the area that is not consistent with our results. 


\subsubsection{Survey Volume}

Assuming a pulse width of $1 \mathrm{~ms}$ (consistent with the ASKAP FRB rate assumption), our nominal fluence limit discussed above corresponds to a peak flux limit of about $12 \mathrm{Jy}$. Adopting the standard candle model discussed by Lorimer et al. (2013) which gives a peak flux-redshift relationship (see their Equation 9), we find a maximum redshift reached by GBTrans to be approximately $z=0.3$. Given the beam solid angle computed in the previous section, the comoving volume corresponding to this limit assuming a standard set of cosmological parameters for a flat universe (Bennett et al., 2014) is $124,000 \mathrm{Mpc}^{3}$. As expected, this is substantially less than what Foster et al. (2018) reported $\left(z=3.3\right.$ and $\left.600,000 \mathrm{Mpc}^{3}\right)$ for the more sensitive ALFABURST survey even with its smaller field of view. We note here that due to the limited sensitivity of GBTrans, the survey volume is significantly smaller than what we would naively expect from searching out to $\mathrm{DM}=9900 \mathrm{pc} \mathrm{cm}^{-3}$. For DMs dominated by intergalactic dispersion, the implied redshift for this DM is about $z=9$. Our choice of searching to such a high DM value is, therefore, very conservative but it mitigates against additional dispersion from FRBs buried in high-density environments with significant amounts of ionised plasma. Future discoveries of highly dispersed FRBs along with directly-measurable redshifts will be able to better quantify the local environments in these sources. 


\section{Chapter 5}

\section{A search for FRBs with the Arecibo telescope}

In the previous chapter, I described GBTrans - a commensal survey for FRBs using a small (20-m) radio telescope. In this chapter, I detail a complementary survey ALFABURST - which makes use of the much larger $305 \mathrm{~m}$ Arecibo radio telescope in Puerto Rico. Initial results of this work were presented by Foster et al. (2018). I describe this experiment here and present the latest results.

\subsection{Introduction}

Among the radio telescopes that currently are undertaking surveys for transient signals, the Arecibo telescope in Puerto Rico is the most sensitive single dish after FAST (Five hundred meter Aperture Spherical Telescope) in China, which is still in its commissioning phase at the time of writing. Both of these dishes are sections of a sphere with $305 \mathrm{~m}$ and $500 \mathrm{~m}$ diameters, respectively. Their large surface areas makes them more sensitive than smaller dishes and while dishes like ASKAP have large sky coverage, single-dishes like Arecibo make it possible to detect FRBs even in their narrow beam's sidelobes.

The ALFABURST survey is a commensal FRB search backend on the Arecibo L-band $^{1}$ Feed Array (ALFA) receiver at the Arecibo Observatory since July 2015.

\footnotetext{
${ }^{1} \mathrm{~L}$-band is a radio astronomy term inherited from WWII and refers to the $1-2 \mathrm{GHz}$ band.
} 
It is a component of the SETIBURST backend previously deployed at the Arecibo telescope (Chennamangalam et al., 2017). The ALFABURST observing system as well as the survey parameters are described in section 5.2. In section 5.3, I present the survey results and consider possible explanations for the non-detection of FRBs in this work so far and examine the consistency of this non-detection with the current FRB sky rates.

\subsection{Observations}

\subsubsection{ALFA Description}

Each ALFA beam is approximately $3.8^{\prime} \times 3.3^{\prime}$ at full-width half maximum. The system temperature of the receiver is $\sim 30 \mathrm{~K}$, and the gain at the central beam is $\sim 11 \mathrm{~K} \mathrm{Jy}^{-1}$, with the peripheral beams having a slightly lower gain. Aside from the seven main beams, the sidelobes of the beams are also sensitive, with the peak of these sidelobes having a loss of only $-8.5 \mathrm{~dB}$, i.e., the gain at the sidelobe peak is only one seventh of that at boresight of the central beam. We account for the sensitivity of the seven ALFA beams all the way out to the outer edge of the first sidelobe as Arecibo would be sensitive to detect most previous FRBs in these, by taking advantage of the large forward gain of the Arecibo dish. We compute this by splitting the beam and first sidelobe into shells of progressively lower gain but larger sky coverage, then integrate to obtain the totals. Fig. 5.1 shows the parameterized ALFA beam model from the Galactic Arecibo L-Band Feed Array (GALFA) HI survey (Peek et al., 2011) at $1425 \mathrm{MHz}$ and clarifies the spatial relationship of the 


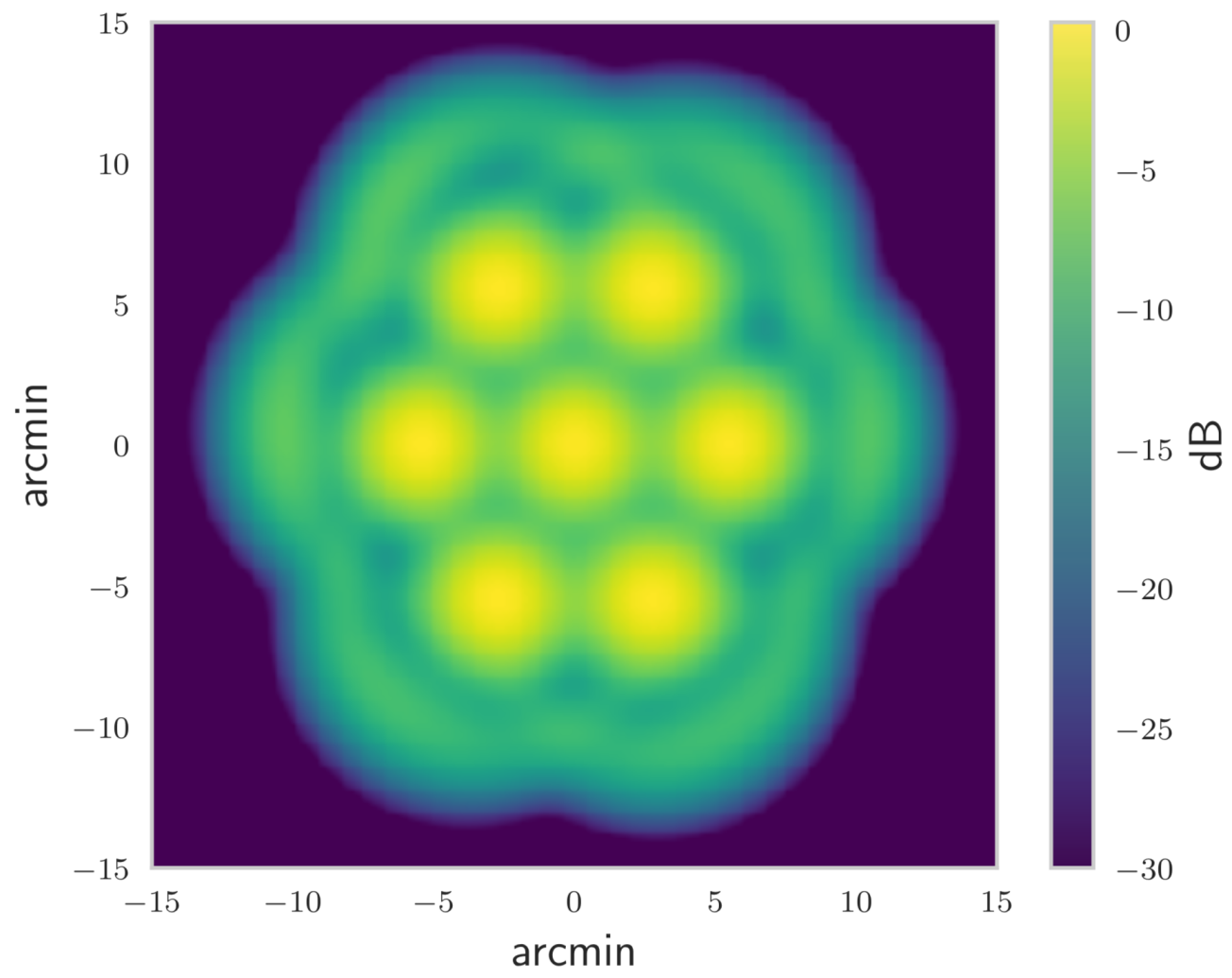

Figure 5.1 Primary and first sidelobe model of the AFLA receiver in decibels, cut-off at $-30 \mathrm{~dB}$. The first sidelobe peaks at around $-9 \mathrm{~dB}$. Image clarifies the spatial relationship of the seven beams and their sidelobe responses. The first sidelobe of any particular beam overlaps the response of the neighboring beams.

seven beams and their sidelobe responses. The first sidelobes peak at around $-9 \mathrm{~dB}$. Accounting for the first sidelobes provides a significant increase in sky coverage compared to just the primary lobes of ALFA.

\subsubsection{ALFABURST description}

The need for commensal observing, wherein multiple data recording/processing processes run simultaneously on data from the telescope during observations, was initiated by SETI (Search for Extraterrestrial Intelligence). SETI is conducting a 
search for signs of technological life, which requires searching a significant amount of sky in data collected with both high frequency resolution and excellent temporal sampling. During commensal observing, the primary observer controls the telescope pointing and overall characteristics of the experiment, while the secondary observers also have access to a copy of the data that they can process independently. Along these lines Chennamangalam et al. (2017) developed 'SETIBURST', a robotic, commensal, real-time multi-science backend for the ALFA receiver whenever it is operated. SETIBURST takes advantage of the wide bandwidth capabilities of a high-resolution analog-to-digital converter paired with an FPGA to digitize, packetize, and transmit channelized time-series voltage data to flexible central processing units (CPUs) and graphics processing units (GPUs). It supports two applications: SERENDIP VI (Search for Radio Emission from Nearby Developed Intelligent Populations), and ALFABURST — the search for FRBs, which is reported here.

The SERENDIP VI spectrometers are installed by the Berkeley SETI Research Center ${ }^{2}$. Their main algorithm is a search for very narrow spectral features drifting in frequency due to the relative acceleration between the Earth and a distant exoplanet. Polyphase filtering (PPF) is a computationally efficient structure for applying resampling and filtering to a signal that leads to building filterbank files. Using a coarse poly-phase filterbank, implemented on FPGAs, the algorithm's wide bandwidth is broken up into manageable chunks (mostly a power of 2), and then the bandwidth is divided using a high resolution poly-phase filterbank implemented on commodity CPU/GPUs. Analog signals from all seven beams are digi-

\footnotetext{
${ }^{2}$ https://seti.berkeley.edu
} 


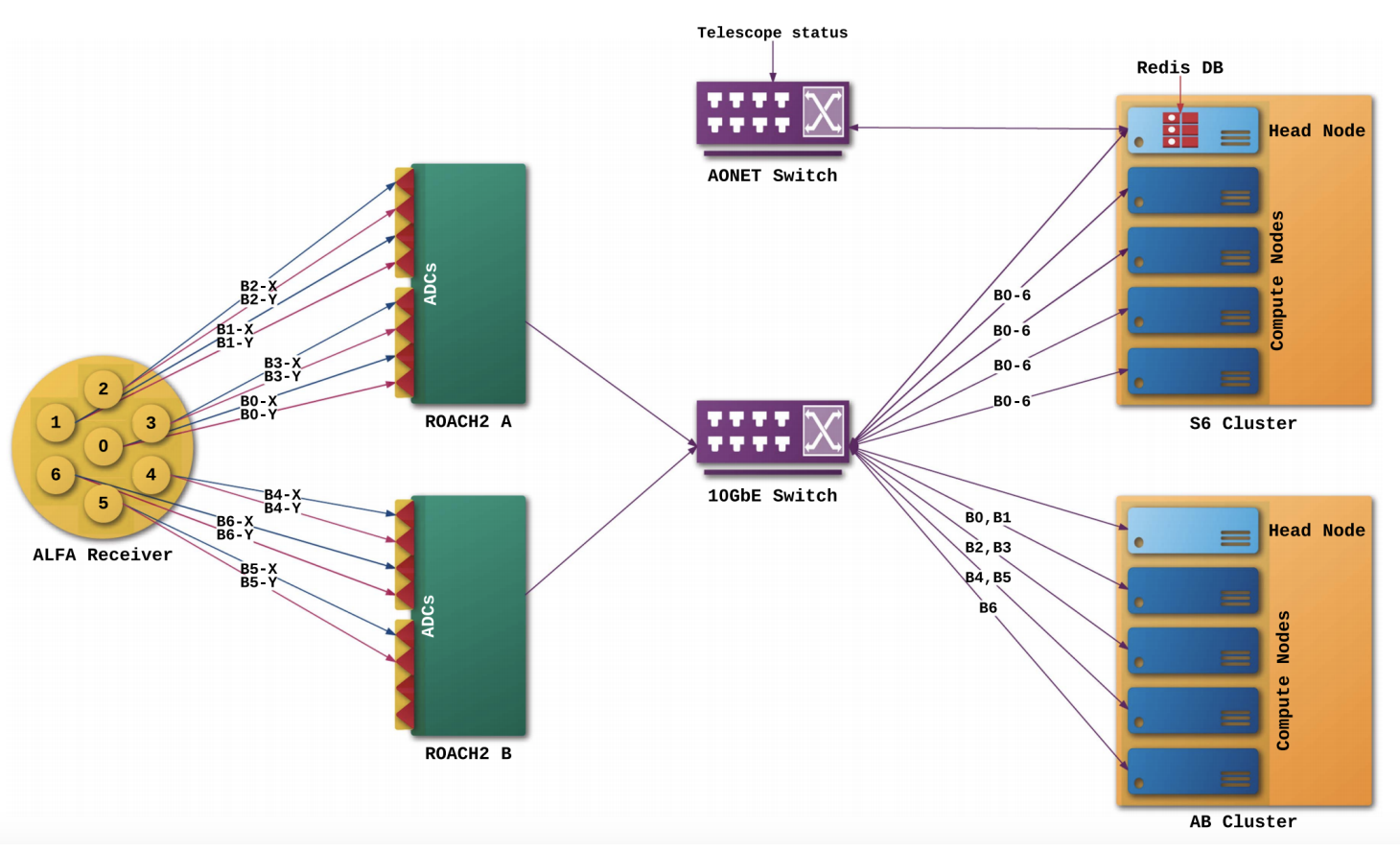

Figure 5.2 Simplified architecture of the SETIBURST system. Both polarizations (denoted by $\mathrm{X}$ and $\mathrm{Y}$ ) of all ALFA beams (B0 - B6) are processed by two ROACH2 FPGA boards and distributed to compute nodes through a broadband switch. "AB" and "S6" stand for ALFABURST and SERENDIP VI respectively in this diagram. (Taken from Chennamangalam et al. (2017))

tized using high-speed converters and fed through a filterbank file and distributed to SERENDIP VI compute nodes via an ethernet switch. As shown in Fig. 5.2, the SERENDIP VI architecture includes a cloning of the resulting spectra so ALFABURST data can be searched for FRBs.

ALFABURST uses ARTEMIS (Advanced Radio Transient Event Monitor and Identification System) software developed for a survey at the UK station of the LOFAR telescope (Karastergiou et al., 2015) as a GPU solution for automated, real-time pulse detection. Fig. 5.3 shows an example search output from ARTEMIS Modular Pelican Pipeline (AMPP) software which consists of a number of $\mathrm{C}++$ modules and configuration files for processing real-time data, including an implementation of a 
poly-phase filter for raw telescope data, a raw data to Stokes parameters converter, RFI rejection, dedispersion, and streaming modules that record data to disk and send the processed data via the network for further processing or recording. AMPP also provides the functionality to store buffers of data for further offline processing. This design allows data processing tasks to be implemented independently from data acquisition, transport protocols, data output, and system deployment. ARTEMIS has four compute nodes that run the ALFABURST software data acquisition pipeline. Three of the nodes process data from two beams each, and the remaining node processes the seventh ALFA beam. The architecture of the software follows a client-server model, where the server receives data through a broadband (10 Gbps) switch and processes a total bandwidth of $\sim 3.2$ Gbps of 16-bit beamformed data. The server fills data corresponding to missing packets with zeros and forward the data to the client. The client is modular by design, with each module handling one logical signal processing stage.

The ALFA data are channelized into 4096 spectral channels for each of the seven beams and full-Stokes spectra are gathered for $\sim 100 \mu$ s at 32-bit precision and streamed via ethernet to the seven GPUs running the ARTEMIS software. Although only total power data are searched, full polarization information are saved in a circular buffer that can be triggered to write the events to disk. The software is currently implemented to process $56 \mathrm{MHz}$ bandwidth from each of the seven beams of the ALFA receiver with a sampling time of $256 \mu \mathrm{s}$. The pipeline is designed to obtain the dynamic spectra from all seven beams and subjects them to inline RFI clipping. Then the recorded $8.4 \mathrm{~s}$ data buffers are de-dispersed over trial DMs 


\section{serial pipeline}

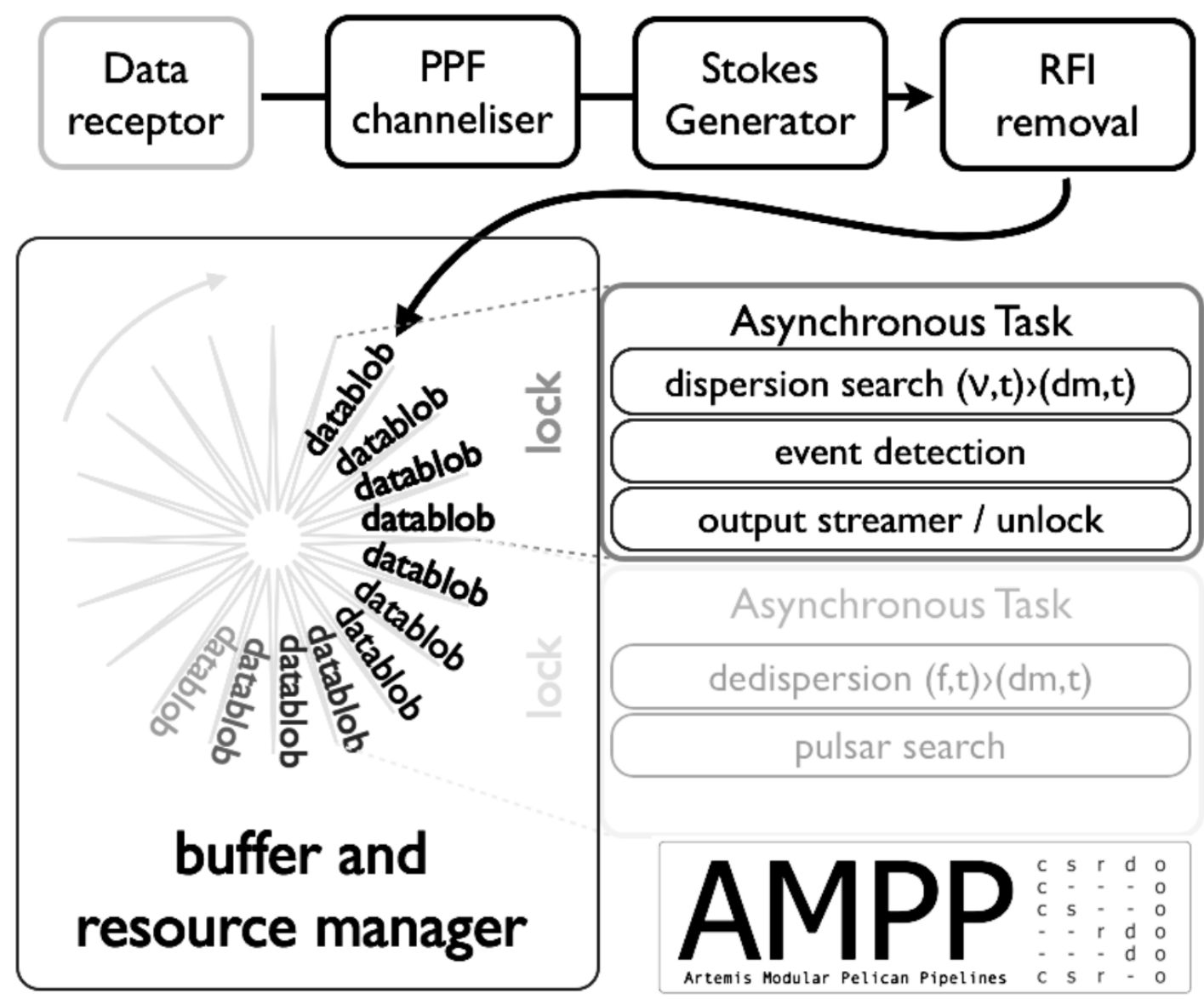

Figure 5.3 Sketch showing the ARTEMIS Modular Pelican Pipeline (AMPP) software. Figure taken from Karastergiou et al. (2015). 
ranging from 0 to $10^{4} \mathrm{~cm}^{-3} \mathrm{pc}$, followed by smoothing to factors of $2^{16}$ in powers of 2 .

\subsubsection{Radio Frequency Interference Excision}

As evidenced previously in pulsar searches, terrestrial radio frequency interference (RFI) is a major obstacle to the identification of celestial radio transients. RFI poses a significant problem, especially at Arecibo, both due to the noisy environment and the high sensitivity of the telescope. Although the inverse frequency squared dispersion provides an excellent discriminator against a number of sources of RFI, the variety in time scale and frequency structure of interfering sources produces many false-positive events. In case of multi-beam receivers though, there is an additional mechanism in order to distinguish astrophysical sources from RFI. Terrestrial radio sources enter radio telescopes through the sidelobes of the antenna, so the RFI sources will appear to be detected at approximately the same intensity in many or all of the receiver beams. Astrophysical sources enter the telescope near a beam axis and should show up strongly in only a single receiver beam. Therefore, we can filter out a significant amount of artificial signals by comparing detections across beams. In ALFABURST, events seen in more than 3 beams simultaneously are rejected from the system, and the filterbank files of the final candidates are recorded with the full polarization dynamic spectra to a local disk for further investigation. If multiple events were detected in the same time window, these events were pooled together and recorded to disk. 
In addition to multibeam RFI rejection, to mitigate against strong RFI sources, an inline RFI exciser is implemented in the pipeline prior to any bandpass correction, time decimation, and integration, which results in a significant reduction in the number of false-positive detections after de-dispersing the data. The following conditions were determined from processing early data sets and making empirical choices to minimize the number of candidates that were produced but clearly caused by RFI. In cases when the power of an individual frequency channel exceeds a threshold $T_{\text {chan }}=5$, it will be replaced with Gaussian noise with zero mean and unit standard deviation $(\mu=0, \sigma=1)$. Moreover, when the frequency-integrated power exceeds a threshold $T_{\text {spectra }}=10$, the entire spectrum will be clipped. As a result, the replaced frequency channels will incorrectly reduce the overall flux for very bright pulses. For ALFA receiver, frequency channels with flux greater than 2.8 Jy and and time samples with frequency-integrated flux greater than $\sim 250 \mathrm{mJy}$, are excised. This might result in the peak of bright FRBs such as FRB 181017 and FRB 180430 to be significantly clipped. However, they would still be detectable as the edges of their pulses would not be clipped.

The zero-DM removal and spectral replacement affects single pulses from lowDM pulsars such as B0834+06. Such pulsars are often clipped by the exciser, but are still detected at significant peak S/N. Since the ALFABURST survey is focused on detecting high-DM pulses, spectral replacement for low DMs does not affect the survey sensitivity. 


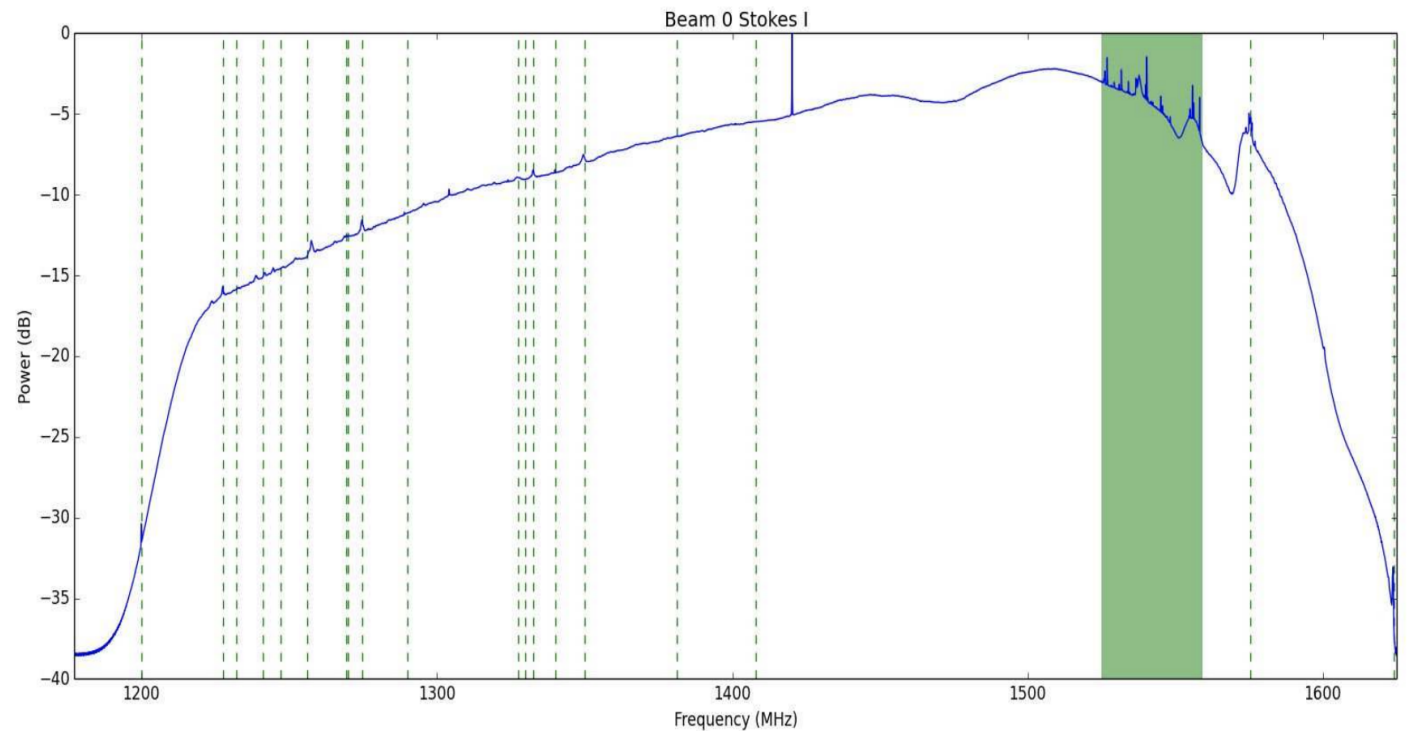

Figure 5.4 Bandpass obtained from a five minute observation of ALFA's central beam using ALFABURST. The dashed vertical lines and the shaded region are the known sources of RFI. The bandpass was also scrutinized to identify known sources of RFI. The peaks in the bandpass matched with the known RFI sources confirming that the obtained band from the instrument was correct.

\subsubsection{Commissioning of ALFABURST}

Validation tests were performed during the commissioning phase of the ALFABURST hardware by generating a bandpass from an on-sky test (Rajwade et al., 2016) which is shown in Fig. 5.4). After testing the bandpass, a few bright pulsars were selected for real-time observations and the ARTEMIS pipeline was tested. These included PSR B0611+22 and PSR B0531+21 (the Crab pulsar) in each of the beams of the ALFA receiver for a span of $\sim 120$ seconds. These observations were also helpful for testing the beam backend mapping of the instrument to ensure the pulsars are detected in the expected data stream. Observations in commensal mode were also performed to test the the pipeline when ALFA is enabled and disabled. 


\subsubsection{Post-processing Pipeline}

As explained in Section 5.2.3, in the processing pipeline the brightest RFI is clipped and replaced, but low-level RFI and spectra statistics still lead to an excess of false-positives. After applying this threshold, we discard less than $5 \%$ of the observed date to RFI.

After the buffers were stored by the ARTEMIS pipeline for further offline processing on the compute nodes, we applied a post-detection pipeline where the data were processed using the HEIMDALL ${ }^{3}$ single-pulse software package. Understanding the features has led to the development of a number of simple filters to reduce the number of false-positive detections before relying on the neural network models. Candidates were filtered out if the minimum DM in each buffer was less than $50 \mathrm{~cm}^{-3}$ pc. Given the optimal DM $\left(\mathrm{DM}_{\mathrm{opt}}\right)$ obtained from the $\mathrm{S} / \mathrm{N}$-maximized $\mathrm{DM}$ trial, if the $\mathrm{DM}$ range exceeds $\left(0.5 \times \mathrm{DM}_{\mathrm{opt}}, 1.5 \times \mathrm{DM}_{\mathrm{opt}}\right)$, then the event is due to long duration RFI. The optimal $\mathrm{S} / \mathrm{N}$ threshold required for detection was set to be 10 in order to eliminate a large fraction of spurious events, while minimizing the likelihood of missing a potential astrophysical signal, and the maximum boxcar width was set to $65.54 \mathrm{~ms}$. The variety of RFI signals and overall commensal nature of this survey may cause unexpected changes in the observing frequency band following by packet loss and incorrect digital gain settings. Therefore, the output from HEIMDALL still contains a large number of false detections. FRB candidates were recorded over a timespan of $10500 \mathrm{~s}$ with at least one detection above the minimum peak $\mathrm{S} / \mathrm{N}$ threshold of 10 . Therefore, we used FETCH in the pipeline (see

\footnotetext{
${ }^{3}$ https://sourceforge.net/projects/heimdall-astro
} 
section 3.3.4) to reduce the number of false positives. For each output candidate, a diagnostic plot was generated that contained the frequency-collapsed time series of the detection, the de-dispersed dynamic spectrum using the S/N-maximized DM, and the DM-time dynamic spectrum. There were 26 final diagnostic plots which were inspected visually, out of which 17 were due to RFI or corrupted data and the rest were inspected further and discussed in section 5.3.2.

\subsection{Results and Discussion}

\subsubsection{Previously published results}

Here I summarize the previous results of ALFABURST survey published in Foster et al. (2018). From July 2015 to August 2017, 125k event windows were recorded corresponding to total 292 hours of observation. These include regular observations of known pulsars scheduled by PALFA to test their survey pipeline that were detected in ALFABURST data. Once we visually confirmed real events, we confirmed if any originated from known sources by obtaining the pointing of the beam that the pulse was found in and checking it against the ATNF pulsar catalog (Manchester et al., 2005). Single pulses from B1859+03, B1900+01 (see Fig. 5.5), B2002+31, B1933+16, among others detected primarily in observations carried out as part of the PALFA survey (Cordes et al., 2006) were detected. Detections of 17 known pulsars among their parameters are listed in Table 5.1. In the new pipeline that was developed as part of work in this chapter though, most pulsar candidates were filtered out by imposing a minimum DM threshold in order to only focus on 


\begin{tabular}{llllll}
\hline \multicolumn{1}{c}{ PSR } & $\begin{array}{c}\mathrm{S}_{1400} \\
(\mathrm{mJy})\end{array}$ & $\begin{array}{c}\mathrm{DM}_{\mathrm{cat}} \\
\left(\mathrm{pc} \mathrm{cm}{ }^{-3}\right)\end{array}$ & $\begin{array}{c}\mathrm{DM}_{\text {obs }} \\
\left(\mathrm{pc} \mathrm{cm}{ }^{-3}\right)\end{array}$ & $\mathrm{N}_{\text {pulses }}$ & $\mathrm{S} / \mathrm{N}_{\max }$ \\
\hline $\mathrm{B} 0525+21$ & 9.0 & 50.87 & 50 & 1 & 72.3 \\
$\mathrm{~B} 0540+23$ & 9.0 & 77.70 & 77 & 1 & 11.7 \\
$\mathrm{~B} 0611+22$ & 2.2 & 96.91 & 101 & 5192 & 48.8 \\
$\mathrm{~J} 0631+1036$ & 0.9 & 125.36 & 125 & 7 & 10.2 \\
$\mathrm{~B} 0834+06$ & 4.0 & 12.86 & 9 & 223 & 35.0 \\
$\mathrm{~B} 1133+16$ & 32.0 & 4.84 & 7 & 291 & 15.5 \\
$\mathrm{~B} 1737+13$ & 3.9 & 48.67 & 46 & 1880 & 49.4 \\
$\mathrm{~B} 1859+03$ & 4.2 & 402.08 & 402 & 2 & 20.4 \\
$\mathrm{~B} 1900+01$ & 5.5 & 245.17 & 246 & 151 & 35.4 \\
$\mathrm{~J} 1908+0457$ & 0.9 & 360.00 & 352 & 3 & 12.9 \\
$\mathrm{~J} 1908+0500$ & 0.8 & 201.42 & 202 & 160 & 18.5 \\
$\mathrm{~J} 1910+0728$ & 0.9 & 283.70 & 288 & 2 & 10.2 \\
$\mathrm{~J} 1913+0904$ & 0.2 & 95.30 & 97 & 1524 & 44.7 \\
$\mathrm{~B} 1913+10$ & 1.3 & 241.69 & 245 & 2 & 16.1 \\
$\mathrm{~B} 1933+16$ & 42.0 & 158.52 & 154 & 10 & 30.5 \\
$\mathrm{~B} 1937+24$ & $*$ & 142.88 & 146 & 37 & 24.6 \\
$\mathrm{~B} 2002+31$ & 1.8 & 234.82 & 250 & 4 & 27.6 \\
\hline
\end{tabular}

Table 5.1 Parameters for known pulsars detected in the ALFABURST survey from July 2015 to August 2017. The columns from left to right are, pulsar name, mean flux density at $1400 \mathrm{MHz}$, catalog DM (obtained from the ATNF pulsar catalog; Manchester et al. 2005), observed DM of the highest S/N pulse, number of detected single pulses, and maximum single-pulse S/N.

FRB detections.

Although there were no FRBs detected in the first two years of the observations with ALFABURST, we have made a detection of an as yet unknown broad-band (within our band limits) pulse with two clear components at a peak $\mathrm{S} / \mathrm{N}$ of 18 . This pulse occurred only in beam 5 and the peak $\mathrm{S} / \mathrm{N}$ is maximized by de-dispersion using a DM of $281 \mathrm{pc} \mathrm{cm}^{-3}$ and time decimation factor 8 . The main pulse width is approximately $3 \mathrm{~ms}$ and the secondary pulse arrived approximately $20 \mathrm{~ms}$ after the primary pulse. This detection occurred at 04:56:16 UT on 2017, June 18 (corresponding to MJD 57922) during a PALFA observing run. Since the telescope was 

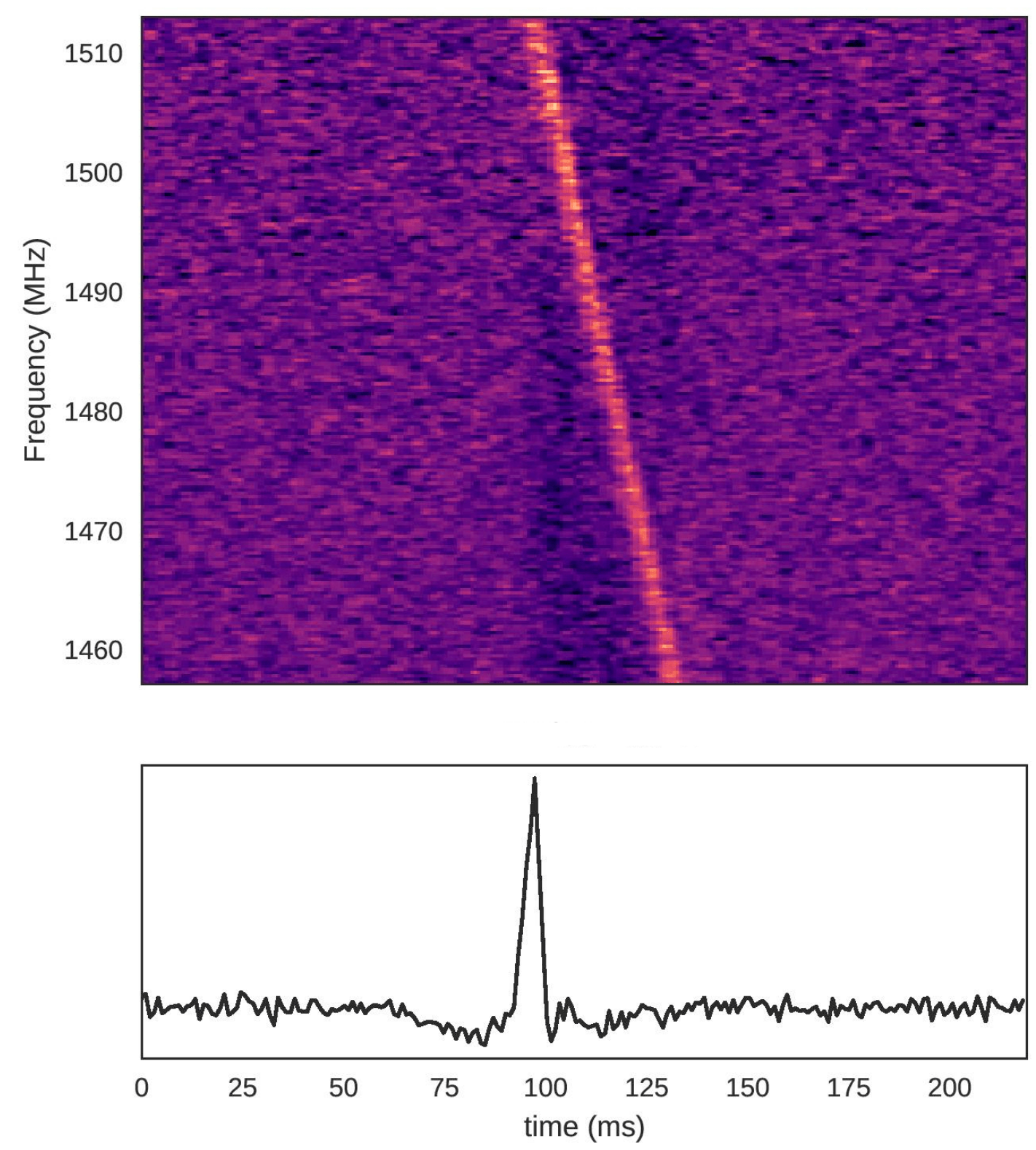

Figure 5.5 Detection of a single pulse from PSR B1900+01 $\left(\mathrm{DM}=245 \mathrm{pc} \mathrm{cm}^{-3}\right)$. The top panel shows the frequency-time spectrum and the bottom panel is the integrated pulse-profile with an arbitrary intensity unit. The baseline dip before and after the pulse is due to zero-DM removal (Eatough et al., 2009). 
slewing between fields, the event was not seen by the PALFA collaboration as the PALFA spectrometers were not designed to run during the slewing times. This event is the first known detection of a transient, broad-band pulse using ALFA during such a slew. However, this makes it challenging to determine an accurate position of the source. During the detection the pointing was changing by approximately 5 arcminutes per second in right ascension (RA) and 2 arcminutes per second in declination (Dec). This slewing rate gives us a conservative estimate of the error in pointing at the time the pulse was detected. Based on the time stamp of the pulse and the pointing data the pulse occurred when beam 5 of ALFA was pointing at RA $18^{\mathrm{h}} 45^{\mathrm{m}} 10^{\mathrm{s}}$ and Dec. $+00^{\circ} 38^{\prime}$. These coordinates are quoted in the J2000 epoch with uncertainty of $20 \mathrm{~s}$ in RA and 2' in Dec. and we tentatively name the transient $\mathrm{J} 1845+00$.

The corresponding Galactic coordinates of J1845+00 are Galactic longitude $\ell=32.78 \pm 0.05^{\circ}$, and Galactic latitude $b=+1.68 \pm 0.05^{\circ}$, which places it along the Galactic plane in the first quadrant. The DM distance estimated in that direction according to the NE2001 model (Cordes \& Lazio, 2002) is approximately $6 \mathrm{kpc}$, which implies a Galactic origin for the pulse. The maximum Galactic contribution along this line of sight would produce a DM of approximately $800 \mathrm{pc} \mathrm{cm}^{-3}$. A search of the ATNF pulsar database (Manchester et al. 2005), Rotating Radio Transient (RRAT) catalog ${ }^{4}$, and recent PALFA discoveries ${ }^{5}$ were performed within one degree

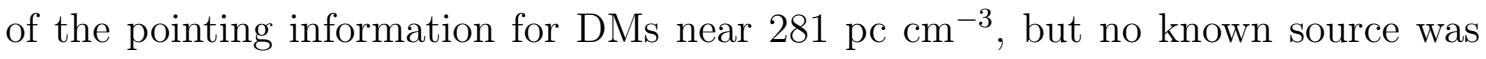

\footnotetext{
${ }^{4}$ http://astro.phys.wvu.edu/rratalog

${ }^{5}$ http://www.naic.edu/palfa/newpulsars
} 
revealed as the result. This region has been surveyed before with PALFA and the Parkes Multi-beam Survey (Manchester et al., 2001), but no significant pulsar was detected at this DM. Fig. 5.6 shows the de-dispersed dynamic spectrum along with the time series for this detection. It is noticeable that the pulse is brighter at higher observing frequencies. This can be either due to scintillation or the fact that telescope was slewing during this time, which causes the telescope gain to change as well. Therefore; the beam gain may be higher at the beginning of the pulse compared to the end of the pulse. Fig. 5.7 shows the DM-time plot of the event. A more detailed analysis of this event and the results of follow-up observations are currently in preparation to confirm if this event is a new RRAT or a pulsar.

Since ALFABURST was installed, the majority of ALFA observation time is allocated for the ongoing Arecibo surveys such as the Arecibo Galaxy Environment Survey (AGES; Auld et al., 2006), the pulsar ALFA survey PALFA (Cordes et al., 2006), and the Galactic ALFA Continuum Transit Survey (GALFACTS; Taylor \& Salter, 2010)). AGES pointings are mostly off the Galactic Plane and therefore, are ideal to a search for FRBs, while PALFA's pointings are emphasizing the Galactic Plane. PALFA has so far resulted in the discovery of 193 pulsars and one FRB (Spitler et al., 2014). Fig. 5.8 shows the sky coverage of ALFABURST for the timespan of about two years. We quantify the depth of the survey metrics by multiplying the instantaneous field of view into the observing time and call it the "survey coverage". The survey coverage of ALFABURST is $\sim 10 \mathrm{deg}^{2}$ hours when accounting for all 7 beams. This is very small compared to the combined Parkes multi-beam surveys with survey coverage of $\sim 4500 \mathrm{deg}^{2}$ hours (Crawford et al., 

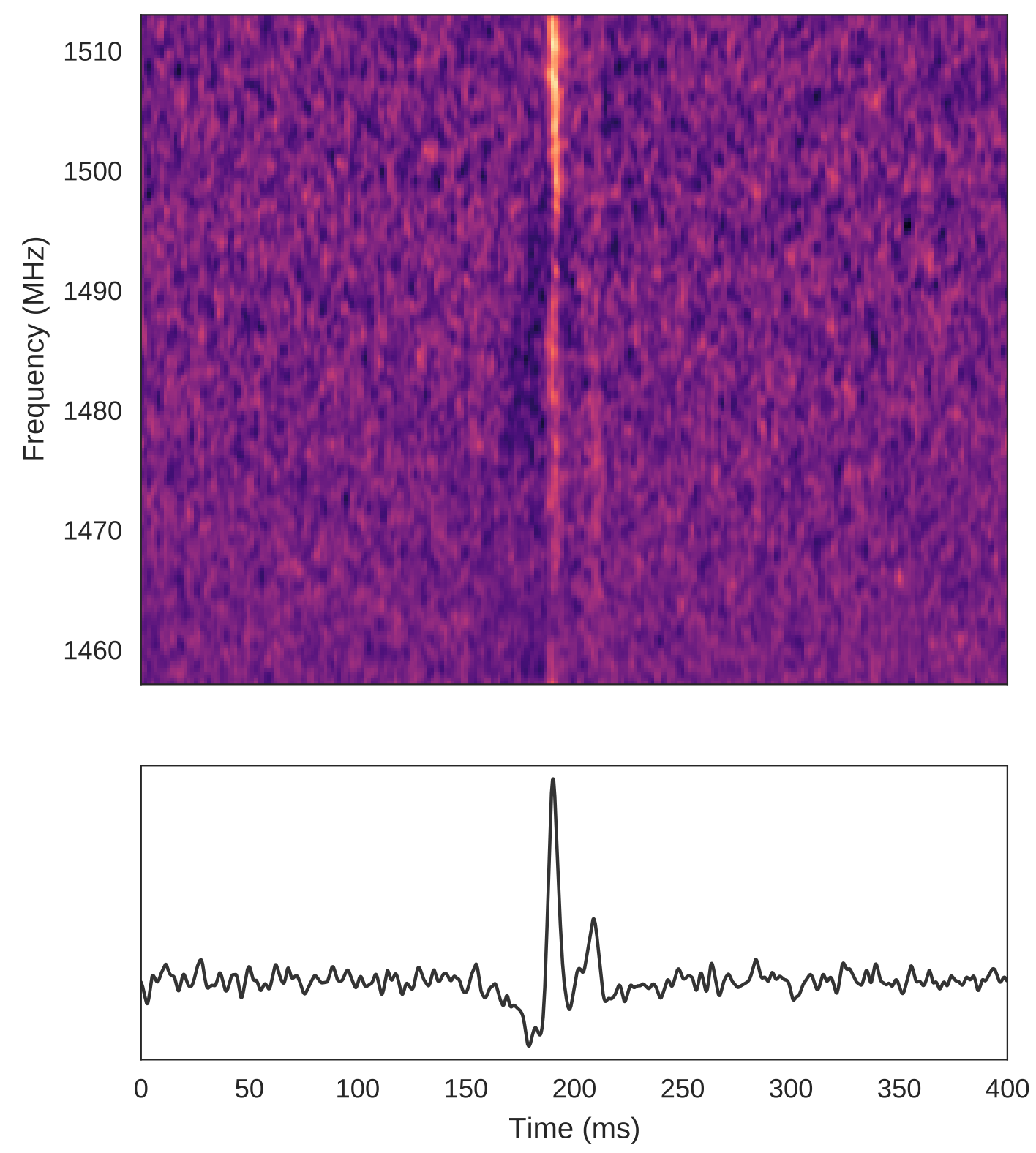

Figure 5.6 Top: A broad band pulse $\left(\mathrm{S} / \mathrm{N}\right.$ maximized at $\left.\mathrm{DM}=281 \mathrm{pc} \mathrm{cm}^{-3}\right)$ detected in beam 5 while the telescope was slewing during a PALFA observation. No known source has been associated with this detection. Bottom: Integrated pulse profile of the event which appears to have two peak components. The main pulse width is approximately $3 \mathrm{~ms}$ and the secondary pulse arrived approximately $20 \mathrm{~ms}$ after the primary pulse. 


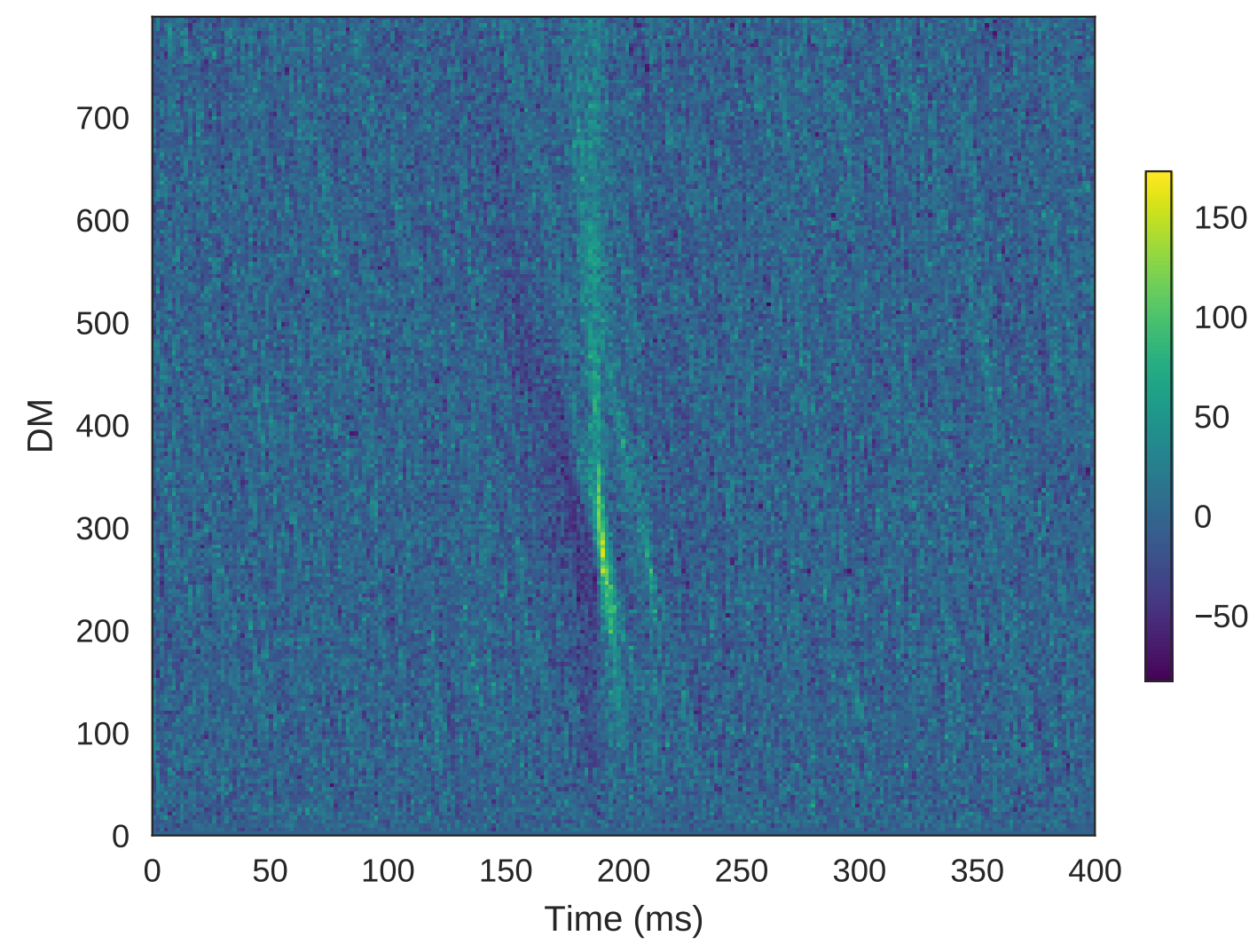

Figure 5.7 DM-time plot of the event shows that the pulse is compact in DM-time space and is consistent with an astrophysical event with $\nu^{-2}$ dispersion relation. The secondary pulse $20 \mathrm{~ms}$ after the primary pulse causes the intensity to be slightly extended to higher trial DMs. 


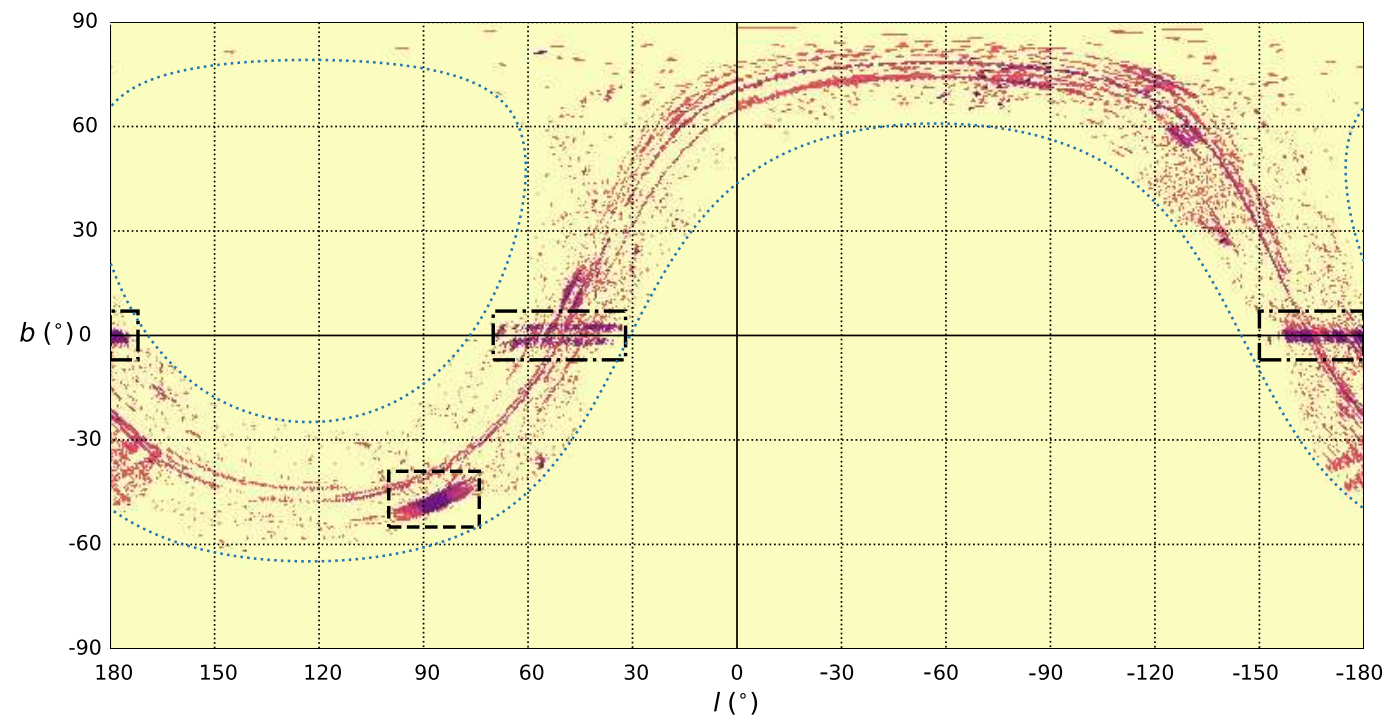

Figure 5.8 Sky coverage during ALFA usage between July 2015 and June 2017, shown in a Cartesian projection in Galactic coordinates along with declination pointing limits (blue dashed). Color represents integration time pointing in a log scale. The majority of ALFA usage during this time was for the PALFA survey along the Galactic Plane (dot-dashed boxes) and the AGES survey (dashed box). The Sshaped arcs across the plot are due to fixed pointings in local azimuth and altitude. As it is clear from the image that the majority of ALFABURST survey time was spent on the Galactic plane for most of our survey.

2016). This is primarily dye to the narrow beam size of Arecibo. ALFABURST compete with other surveys on sensitivity rather than sky coverage. This results in probing a greater redshift range than the surveys done by Parkes.

From the beginning of July 2015 to the end of April 2017 ALFA has been used for approximately 1400 hours of observing and ALFABURST was functional for, on average, 322 hours per beam. The current system is set up to be reliably in use for all beams any time ALFA is active and in the correct receiver turret position. Since April 2017 this stable version of the pipeline has run for an additional 196 hours. This has resulted in a total of 518 hours of processed observing time since 
ALFABURST began commensal observations until August 2017.

\subsubsection{Recent Search Results}

As mentioned in Section 5.2.5, there were 26 final candidates as the output of the post-processing pipeline, which were inspected visually. Among those, 17 were visually confirmed to be due to RFI based on their corrupted-looking spectral features (either narrow or wide frequency band), and the rest were inspected further. Fig. 5.9 shows these pulses and Table 5.2 summarizes their parameters. Unfortunately, due to incomplete metadata during the time of these observations, exact positions of the candidates are not currently known. At the time of writing of this thesis, we are working with Arecibo observatory staff to locate independent telescope log files.

At the present time, with the incomplete data on hand, our working assumption is that the brightest of these pulses is in fact a sidelobe detection of a single pulse from PSR B1915+13, in beam number 5 . This is inferred from a match to the ATNF pulsar catalog to the pulsar's DM $\left(94.5 \mathrm{~cm}^{-3} \mathrm{pc}\right)$ and pulse width $(9.1 \mathrm{~ms})$. With a mean flux density of 6.8 mJy at $1.4 \mathrm{GHz}$, PSR B1915+13 is the only source in the catalog bright enough and close enough in DM to be detectable. The other eight pulses in Table 5.2 have no close DM matches in the catalog and remain viable but as yet undetermined single-pulse candidates. 


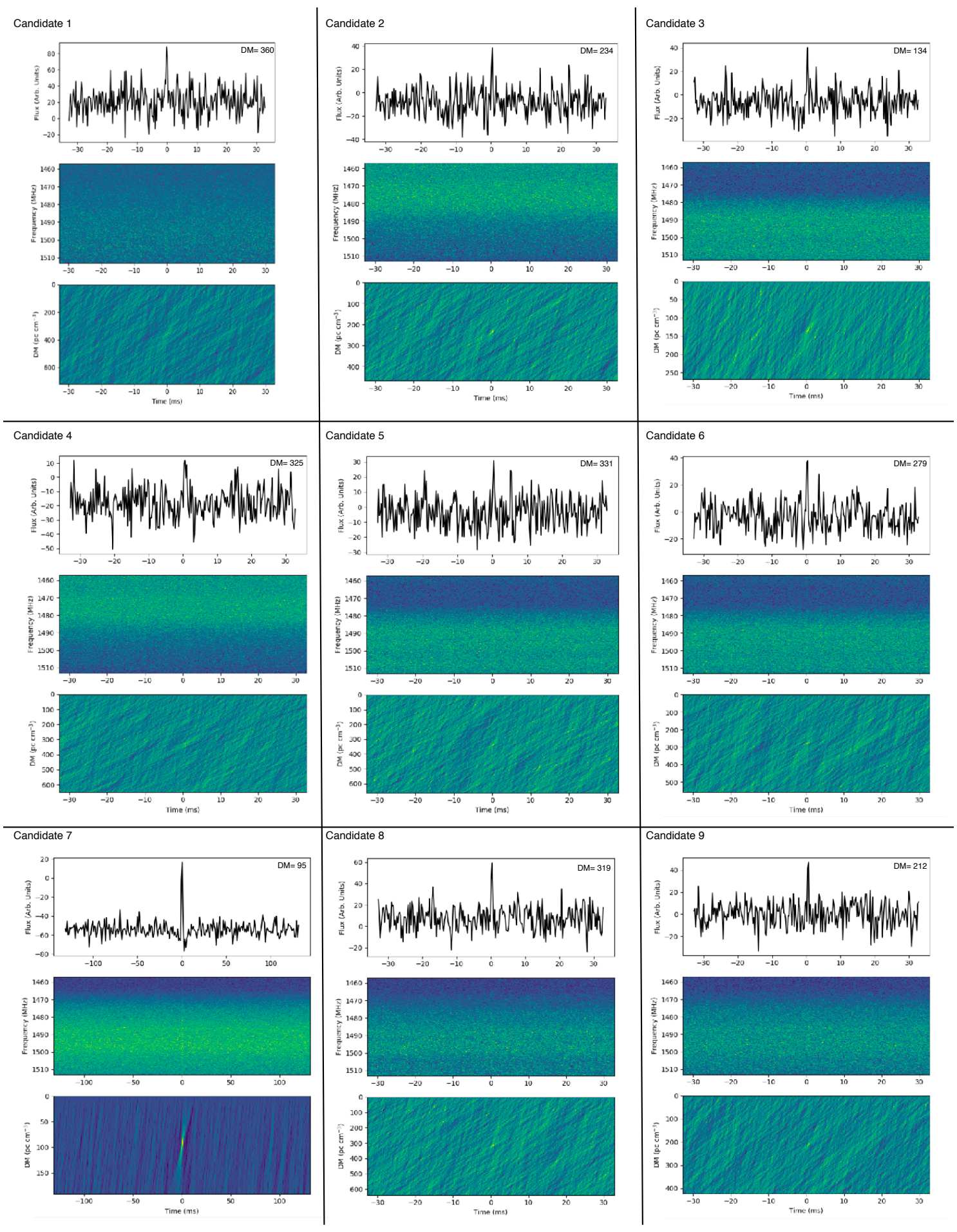

Figure 5.9 Nine transient pulses detected in ALFABURST data. For each candidate the top panel is the de-dispersed time series, the middle panel is the de-dispersed frequency spectrum, and the bottom panel is their corresponding DM-time. Beam number and DM value in $\mathrm{cm}^{-3} \mathrm{pc}$ are labeled on top left and top right of each candidate pixel respectively. 


\begin{tabular}{lcll}
\hline Candidate & MJD & $\begin{array}{c}\mathrm{DM} \\
\left(\mathrm{pc} \mathrm{cm}^{-3}\right)\end{array}$ & $\begin{array}{c}W \\
(\mathrm{~ms})\end{array}$ \\
\hline 1 & 58121.208546 & 360.03 & 0.50 \\
2 & 58073.661026 & 234.04 & 0.25 \\
3 & 58078.065327 & 133.78 & 0.25 \\
4 & 58098.051896 & 325.19 & 0.25 \\
5 & 58078.064848 & 330.97 & 0.25 \\
6 & 58078.065453 & 279.32 & 0.25 \\
7 & 58073.905626 & 95.21 & 2.00 \\
8 & 58085.092596 & 319.42 & 0.50 \\
9 & 58078.067843 & 211.59 & 0.25 \\
\hline
\end{tabular}

Table 5.2 Parameters for the single-pulse detections in the ALFABURST survey. The columns from left to right are, candidate number, MJD of the event, DM, pulse width, and the beam number.

\subsubsection{Survey Sensitivity}

The survey sensitivity is computed using the radiometer equation (see Eq. 3.5) and adopting the nominal parameters of the system (typical system temperature of $30 \mathrm{~K}$, Gain of $11 \mathrm{~K} / \mathrm{Jy}$, bandwidth of $56 \mathrm{MHz}$ ), the minimum fluence was estimated to be $0.006 \mathrm{Jy} \mathrm{ms}$ for a pulse width of $5 \mathrm{~ms}$ with the $\mathrm{S} / \mathrm{N}$ threshold of 10 . The minimum detectable fluence at the full-width half maximum (FWHM) of the central beam of ALFA is therefore about 0.012 Jy ms. Fig. 5.10 shows the ALFABURST sensitivity region based on pulse width and peak flux, assuming detection at central beam, along with the known FRBs for a comparison. It indicates the survey would be able to detect all previously reported FRBs. Bright FRBs such as FRB 150807, FRB 180110, FRB 181017, and FRB 180430 would be partially clipped by the inline RFI exciser (Section 5.2.3), but they would still be detected at a high peak S/N. Additionally, in a multiple beam system a bright FRB would be picked up at a lower 


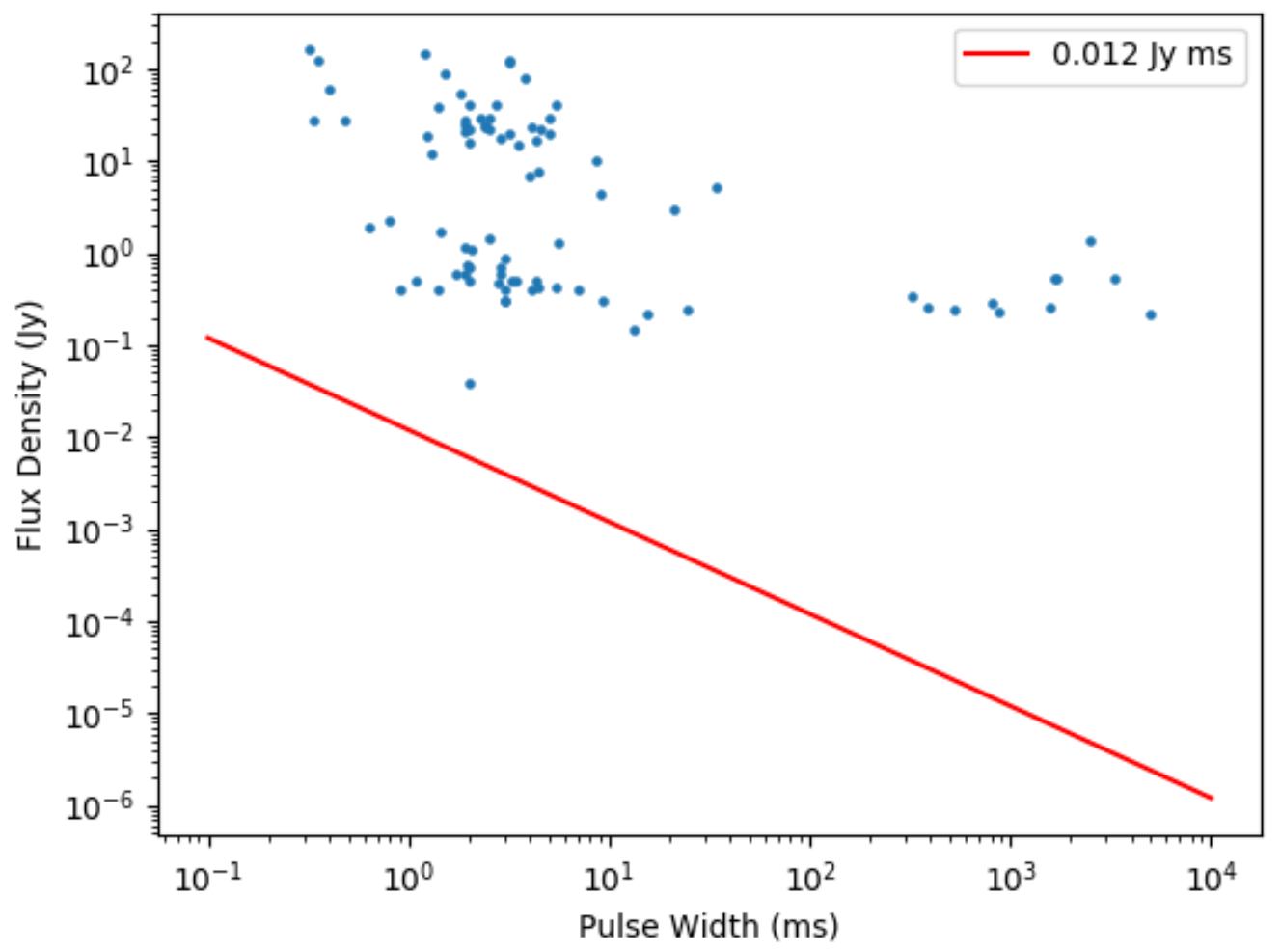

Figure 5.10 ALFABURST single-pulse sensitivity as a function of pulse width. Previously detected FRBs are plotted for reference. The fluence completeness of the survey (red line), determined by the minimum detectable fluence is plotted for reference. The fluence completeness of the survey (dashed) is determined by the minimum detectable fluence at the maximum sampled pulse width in the survey. ALFABURST has a fluence completeness of $0.012 \mathrm{Jy} \mathrm{ms}$ for a pulse widths of $5 \mathrm{~ms}$. The repeating FRBs are plotted with the peak flux density and the pulse width based on the burst with minimum fluence among the rest.

flux in the sidelobes of nearby beams. As of now, the pipeline decimates in time out to $16 \mathrm{~ms}$ and is still sensitive to wider pulses, but at a loss in $\mathrm{S} / \mathrm{N}$.

\subsubsection{Discussion}

Given the piggyback nature of the ALFABURST survey, with each survey observing a different part of the sky for different amounts of time and the deficient 
logging information, and the fact that FRB event rate models and Galactic latitude dependence are not well constrained, it is challenging to come up with a rigorous expectation of the number of detections or constraining the source count index as we did in Section 4.4.1. Specifically, we assume the following criteria:

- a standard candle model (Lorimer et al., 2013);

- the rate of FRBs per host galaxy is independent of redshift;

- a constant galaxy co-moving number density of $10^{-2} \mathrm{Mpc}^{-3}$;

- a flat spectral index;

- a pulse width of $4 \mathrm{~ms}$.

With this, along with measurements of telescope sensitivity and beam size, we find that this survey probed redshifts out to about 3.4 (corresponding to a co-moving distance of $6.8 \mathrm{Gpc}$ and an effective survey volume of around 600,000 $\mathrm{Mpc}^{3}$ ), when using all 7 ALFA beams. The number of galaxies assumed here is approximate and has been averaged over our best understanding of the number density of galaxies with redshift (Vedantham et al., 2016). Considering the possibility of detecting bright FRBs in the sidelobes of the ALFA beam, the sensitivity of our survey increases slightly up to a redshift of 3.5. Based on this, we would expect, at the $99 \%$ confidence level, to see at most two FRBs. Based on the volumetric event rate from Crawford et al. (2016), we adopt $R_{\mathrm{FRB}}$ to be in the range $(1-7) \times 10^{-4}$ FRBs per galaxy per year. With these assumptions, and based on the estimated observing time based on the PALFA and AGES surveys usage of ALFA, our non-detection is expected. 
The standard candle assumption however, which is a simple model based on updates to the empirical event rates from detections in the HTRU survey (Thornton et al., 2013) by Crawford et al. (2016), assumes that FRBs are singular events, and is subject to uncertainty. As shown by recent statistical studies of the Parkes FRBs, there is growing evidence that they are not standard candles, and their event rate is redshift dependent (Caleb et al., 2016; Rane, 2017). The fact that our simple estimate of 0-2 detections so far is broadly consistent with our actual null detection indicates that our results are not highly sensitive to the standard candle assumption. Future studies with larger samples will be more sensitive to the other assumptions in the present analysis (e.g. flat spectra, constant number density and pulse width). We anticipate significant additional progress in this area as a result of current and planned FRB surveys. These and other issues are discussed further in Chapter 6. 


\section{Chapter 6}

\section{Conclusions}

Here I summarize the importance of this thesis's work, put things into perspective, and look ahead to future relevant projects that can be done.

\subsection{GBTrans constraints on Galactic FRBs}

As described in Chapter 4, using GBTrans we have searched for FRBs commensally for over 500 days. The observations were nominally sensitive to FRBs with redshifts out to about 0.3 but none were found. One implication from this non-detection is an upper limit on the bright FRB rate in the Milky Way. If we assume all FRBs have the same luminosity comparable to FRB $121102\left(\sim 10^{33} \mathrm{~W}\right)$, then according to Equation 1.13, the peak flux density of a Galactic burst that is located $3 \mathrm{kpc}$ away and is observed over $100 \mathrm{MHz}$ bandwidth is almost $10^{10} \mathrm{Jy}$. Such a bright pulse would easily be detectable by GBTrans from anywhere within the Milky Way. We can use this to place a constraint on the Galactic FRB rate $\mathcal{R}=n / \Omega T$, where $n$ is the number of bursts detected, the instantaneous solid angle $\Omega=2.2 \times 10^{-4}$ sr or $1.7 \times 10^{-5}$ of the whole sky, and the total observing time $T=503$ days. Setting $n<1$, we find $\mathcal{R}<117$ events per sky per day. Although somewhat constraining, GBTrans was not designed with this putative population in mind. Currently, the best limits on Galactic FRB rates come from the STARE2 
experiment (Bochenek et al., 2020) with $\mathcal{R}<40$ FRBs per sky per year.

\subsection{Source counts revisited}

As discussed in Section 1.1.9, for a Euclidean distribution of standard candles, we expect a source count function $N(>S) \propto S^{-\alpha}$, where the source count index $\alpha=3 / 2$. Our non-detection of FRBs with GBTrans led to an upper limit on the source count index $\alpha<2.5$ with $90 \%$ confidence. To place this result into context, we carried out Monte Carlo simulations of the $\log N-\log S$ distribution for putative FRB populations given various assumptions. The results from these simulations are shown in Fig. 6.1. Assuming FRBs to be uniformly distributed in Euclidean space, we see the $\alpha=3 / 2$ behavior as shown in the blue line (Model A). Also shown is the non-Euclidean case where the sources are distributed uniformly in terms of comoving volume (orange curve; Model B). This more realistic model results in a flatter distribution with $\alpha \rightarrow 1$. Also shown are variations on models $\mathrm{A}$ and $\mathrm{B}$ in which the standard candle model is relaxed. For the purposes of this analysis, we choose a log-normal distribution of luminosities with a standard deviation that is $13 \%$ of the mean and shown as models $\mathrm{C}$ and $\mathrm{D}$ (green and red curves) respectively. As can be seen, these factors change the source count index significantly and even allow for cases where $\alpha<1.0$. While the constraints that we have from GBTrans $(\alpha<2.5)$ are not sufficient to rule out any of these scenarios, this figure illustrates how sensitive the observed source count distribution is to different assumptions on the spatial distribution and luminosity function of FRBs. Ongoing experiments with 


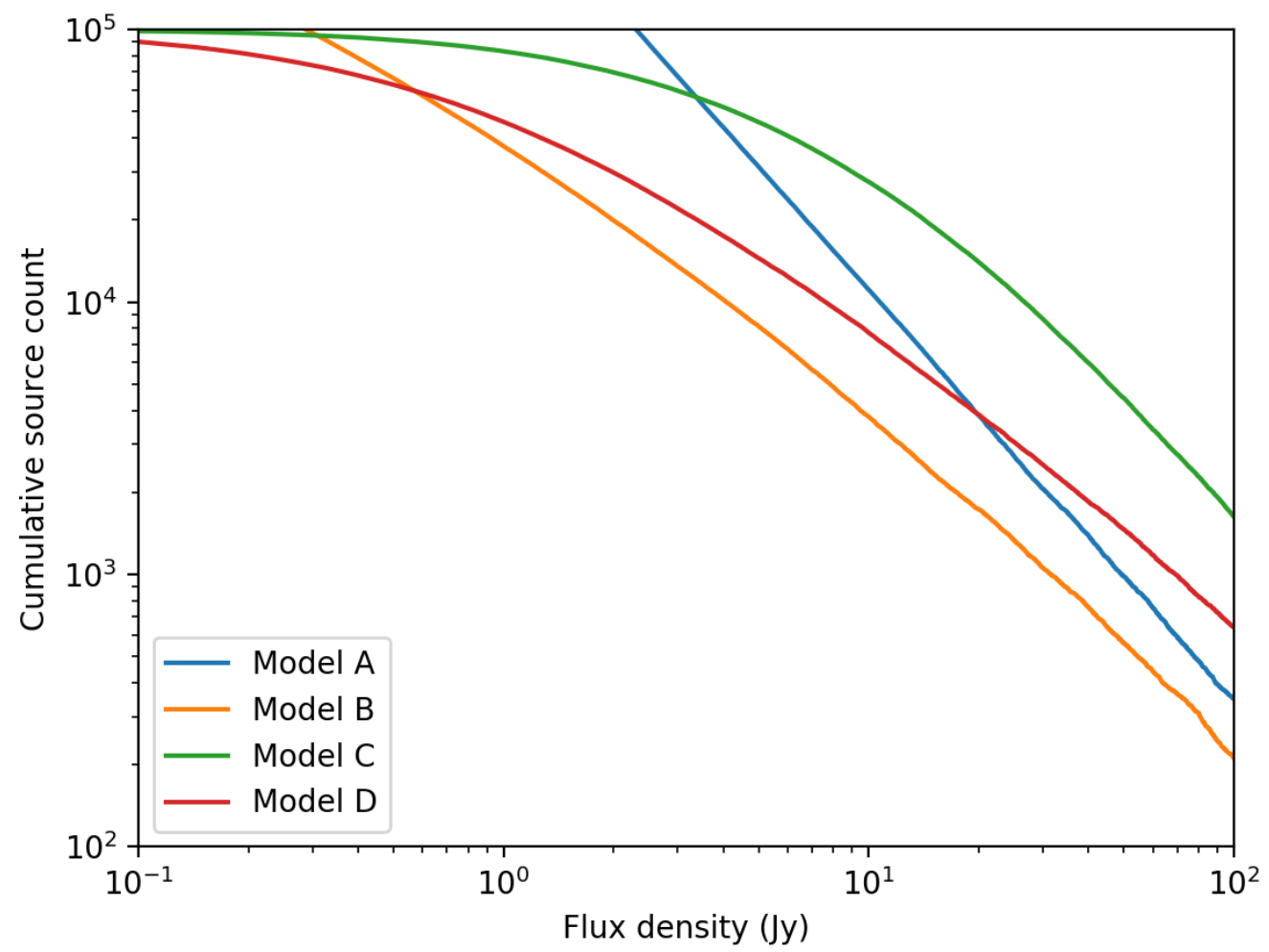

Figure 6.1 Monte Carlo simulations of FRB source counts as functions of flux density constructed under different assumptions. Model A assumes Euclidean distribution of standard candles whereas Model B incorporates cosmological effects of the expanding Universe and assumes standard candles uniformly distributed in comoving space. Model C assumes log-normal luminosity function in Euclidean space and finally Model D assumes the most case of cosmological distribution with log-normal luminosity function.

greater sensitivity such as ALFABURST and, in particular, CHIME are expected to provide great insights into this in the near future.

\subsection{Future FRB searches with $20 \mathrm{~m}$ telescope}

Our detection of numerous pulses from known pulsars has validated the observing system. In addition to a forthcoming publication concerning giant pulses from the Crab pulsar found during the course of this project, future uses of the 20-m 
telescope to search for FRB field are migrating to targeted searches such as the Swift survey described by Gregg et al. (in prep.). Ongoing work described below aims to adapt the system to operate as a rapid response observer of radio transient signals associated with GRBs.

The existence of the repeating FRBs suggests that at least some FRBs are produced in non-cataclysmic events. Multi-wavelength approaches, although difficult, hold the key to understanding the origin and nature of FRBs as was the case for gamma-ray bursts (GRBs). DeLaunay et al. (2016) proposed the first discovered gamma-ray transient associated with an FRB (FRB 131104), in a correlation study between Swift observations and catalogued FRBs. Before that, Bannister et al. (2012) reported two low-significance radio pulses detected between 500 and 1000 seconds after two GRB events. No further coincidences were found in a similar follow-up study done by Palaniswamy et al. (2014).

Zhang (2013) proposed the primary motivation for studying the correlation between FRBs and GRBs is to investigate the possible connection between FRBs and GRBs based on the "blitzar" model (see Section 2.4). He suggests that a small fraction of FRBs can be physically connected to GRBs as FRBs can happen in supra-massive neutron stars collapsing into a black hole shortly after their births, and may have been detectable in X-ray afterglows of some long and short GRBs. He therefore proposes a prompt radio follow-up observations of GRBs starting $100 \mathrm{~s}$ after GRB triggers.

The 20-meter telescope at GBO is fully equipped and available for dedicated monitoring to target up as many as 100 GRB sources according to the telescope's 


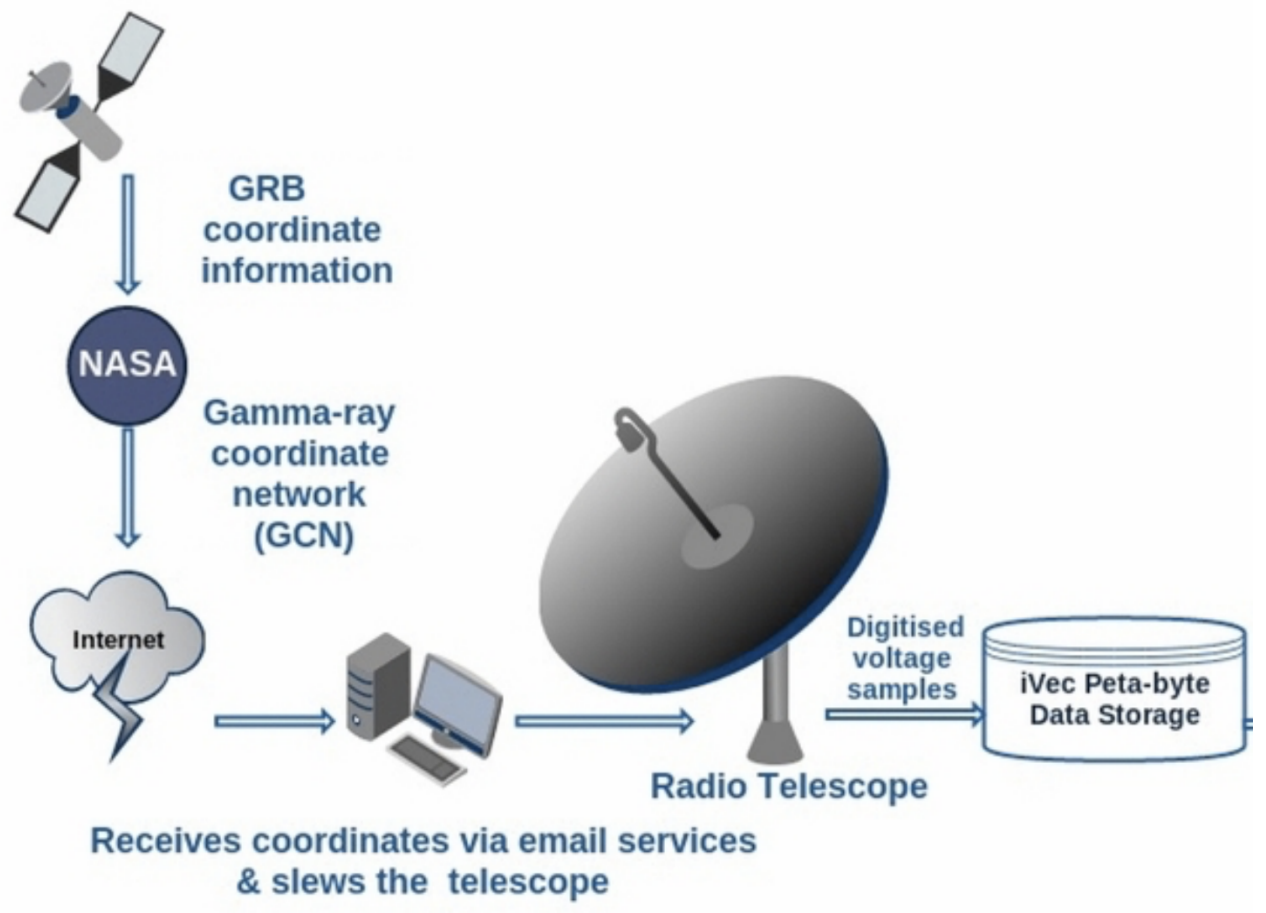

Figure 6.2 Cartoon of the triggered GCN/TAN burst alert system which will be implemented on the 20-m telescope. A GRB event (top left star) occurs somewhere in the sky and is detected by one of the existing high-energy telescopes. The coordinates are sent to the $20-\mathrm{m}$ dish and is brought into action by an autonomous script. Image Credit: Divya Palaniswamy.

sky access and rapid response. In addition to target observations, data collected commensally by the GBTrans pipeline will enable further discoveries. Together with the Gamma Ray Coordinates Network (GCN) and the Transient Astronomy Network (TAN) by Barthelmy (2015), an autonomous alert system shown in Fig. 6.2 will be implemented.

Current network statistics based on GBTrans results shows that the telescope is available about $80 \%$ of the time. It is anticipated that for an isotropic distribution, almost 150 GRBs over the whole sky will be detected over the course of three years. 
Given that for the latitude, up to $80 \%$ of the sky is observable, a sample of up to 100 sources is a more realistic number to expect during this timescale. Depending on visibility, each source will be observed for up to two hours to search for pulses produced on timescales of a few thousand seconds after each GRB. Taking advantage of the telescope's $2 \mathrm{deg} \mathrm{s}^{-1}$ slew rate in both axes, the implementation will check telescope availability and slew time first and only go to those sources that it can reach 2-3 minutes from receiving the trigger. This project would build upon GBTrans nondetection discussed in Chapter 4 to further constrain the source count function for FRBs.

\subsection{ALFABURST}

In Chapter 5, I summarized the implementation, initial operations and results, as well as the recent results of a commensal search for FRBs using the Arecibo telescope's ALFA receiver. Given the uncertainties in the physical characteristics of known FRBs, this non-detection result is broadly consistent with the expectations from a simple model in which FRBs are treated as flat-spectrum standard candles uniformly distributed per unit co-moving volume.

Due to the fact that a number of surveys carried out with ALFA have come to an end, the usage of the ALFA receiver has been decreased over the time period ALFABURST has been active. The survey results obtained from the designed pipeline

developed as part of this thesis increased ALFABURST's survey time and resulted in one likely detection of PSR B1915+13 as well as eight further single-pulse candi- 
dates with DMs in the range $134-360 \mathrm{~cm}^{-3}$ pc. The current ALFABURST pipeline is undergoing an upgrade for the real-time exciser to reduce the false detection rate, which will significantly ease the follow-up and analysis cycle. As part of this upgrade, the RFI-clipper will be trained with a machine learning algorithm to learn the bandpass correction, and high $\mathrm{S} / \mathrm{N}$ events will be filtered. Moreover, before the retirement of ALFA, sampling a larger portion of frequency space for ALFABURST survey from the current input bandwidth of $56 \mathrm{MHz}$ (due to IO limitations) to the full $336 \mathrm{MHz}$ digital bandwidth will be done. This will improve the detection rate limits for scintillating or low-fluence FRBs according to the increased survey sensitivity. More on-sky time in future is expected to result in the detection of several FRBs and consequently testing the validity of our assumptions about their population.

\subsection{Current FRB searches with Arecibo and the GBT}

As discussed in Section 6.3, the relationship between FRBs and GRBs is being studied widely. Large single dish telescopes with high sensitivity such as Arecibo and the GBT are well-suited for such targeted searches and should allow the repeating bursts to be detected up to a redshift of $z \approx 2$. In an experiment I have participated in being led by Palliyaguru et al. (in prep.), we present a non-detection search results for potential FRBs at 11 well-localized GRB sources, detectable at Arecibo and GBT and all away from the Galactic plane, that show evidence for the birth of a magnetar at frequencies of 1-5 GHz. The target list includes six GRB-SNe, 
four short GRBs and one long GRB without a SN association. These searches were conducted $\sim 1-15$ years after the explosions. The searches resulted in candidates that were confirmed to be either RFI or single pulses from the known test pulsars but no FRB candidates were found. The target list is summarized in Table 6.1. Soft component in the X-ray up to $10000 \mathrm{~s}$ is thought to be associated with an X-ray flash rather than a typical GRB suggestive of the collapse to a NS rather than a BH. All these sources have been localized to a region $<2.5$ arcsec by high-energy observations, which allows single dish telescopes with a field of a few arcmins to cover the entire error area. All observations were carried out within a timespan of 412 days.

Arecibo observations occurred between December 12, 2017 and December 2018 on epochs listed on Table 6.2. A total of 114 hours of observations were obtained on all GRBs, with $\sim 1-21$ hours on each target at each frequency depending on the availability. GBT observations were carried out between November 2, 2017 and July 31, 2018 on 10 epochs. To mitigate against any possible self-absorption at early epochs, observations were conducted at 1.4 and $1.9 \mathrm{GHz}$ on the first two epochs and afterwards only at $1.4 \mathrm{GHz}$ on the following epochs.

Using the radiometer equation (Eq. 3.5), the minimum detectable flux density at the full-width half maximum (FWHM) of the ALFA beam is $30 \mathrm{mJy}$. The luminosity distance for each source $D_{L}=(1+z) D(z)$, where $D(z)$ is the co-moving distance of the source and $z$ is the corresponding redshift. Having that and assuming for simplicity that the sources are isotropic emitters we have $L_{\min }=4 \pi S_{\min } D_{L}^{2}$ as the minimum detectable luminosity for each GRB source. 
Table 6.1 GRB-SNe and GRBs that show X-ray plateaus (Dainotti et al., 2017) detectable at Arecibo. From left to right, we list name, redshift, distance, error radius, RA, DEC, Galactic position, expected DM, and the expected radio flux from an FRB at the GRB location (The measured fluxes from the FRB 121102 were scaled to the distance of each GRB to estimate the expected flux if a repeater-like source resided in the GRB site).

\begin{tabular}{llllllll}
\hline Name & $z$ & $\begin{array}{l}D \\
(\mathrm{Mpc})\end{array}$ & $\begin{array}{l}r \\
\left({ }^{\prime}\right)\end{array}$ & $\begin{array}{l}\text { RA } \\
(\mathrm{h}: \mathrm{m}: \mathrm{s})\end{array}$ & $\begin{array}{l}\mathrm{DEC} \\
(\mathrm{d}: \mathrm{m}: \mathrm{s})\end{array}$ & $\begin{array}{l}\mathrm{DM} \\
\left(\mathrm{cm}^{-3} \mathrm{pc}\right)\end{array}$ & $\begin{array}{l}S \\
(\mathrm{Jy})\end{array}$ \\
\hline SN2003dh & 0.169 & 812 & 0.08 & $10: 44: 50$ & $21: 31: 18$ & 264.32 & 0.573 \\
SN2006aj & 0.033 & 145 & 2.5 & $03: 21: 39$ & $16: 52: 01$ & 173.08 & 17.97 \\
SN2012bz & 0.280 & 1434 & 2.0 & $09: 07: 38$ & $14: 01: 06$ & 413.64 & 0.183 \\
SN2013cq & 0.340 & 1795 & 1.0 & $11: 32: 32$ & $27: 41: 51$ & 472.37 & 0.117 \\
SN2013dx & 0.145 & 687 & 0.5 & $14: 29: 14$ & $15: 46: 26$ & 241.17 & 0.801 \\
SN2013ez & 0.597 & 3508 & 1.5 & $02: 53: 56$ & $13: 23: 13$ & 991.80 & 0.03 \\
130603B & 0.356 & 1894 & 1.0 & $11: 28: 48$ & $17: 04: 16$ & 498.47 & 0.105 \\
140903A & 0.351 & 1863 & 1.0 & $15: 52: 03$ & $27: 36: 09$ & 496.38 & 0.108 \\
GRB051221 & 0.547 & 2868 & 1.0 & $21: 54: 48$ & $16: 53: 28$ & 786.02 & 0.045 \\
GRB100816A & 0.803 & 4529 & 1.0 & $23: 26: 57$ & $26: 34: 43$ & 1396.40 & 0.018 \\
GRB130831A & 0.479 & 2459 & 2.1 & $23: 54: 29$ & $29: 25: 47$ & 663.84 & 0.062 \\
\hline
\end{tabular}

The power-law model for the FRB luminosity function takes the form $\left(L / L_{0}\right)^{-\alpha}$, the rate of bursts above luminosity $L$ can be expressed as $R=R_{0}\left(L / L_{0}\right)^{-\alpha}$, where $\alpha$ is the power law exponent and constant $R_{0}$ is the rate of the bursts brighter than the reference luminosity of $L_{0}$. If a GRB site was searched for $T$ hours, the expected number of pulses for a GRB is, $n=R T$. Because we don't have any detections, it is hard to constrain $R_{0}$ uniquely. However with the different luminosity limits, we hope to constrain $\alpha$ from multiple observations.

Law et al. (2019) submitted a white paper to the Astro2020 Decadal Review discussing radio signatures of magnetar birth, the scientific impact in the next decade and implications for fundamental physics and cosmology. The detection of a latetime FRB signal from a GRB site would definitely be the signature of magnetar birth. Better neural network algorithms that reduce the number of candidates and 
Table 6.2 Summary of the observations and limits obtained so far. From left to right we list the GRB name, observing frequency $(F)$, total time on source $(T)$, the number of epochs observed $(N)$, the average time $(t)$, the luminosity distance $\left(D_{L}\right)$, the redshift $(z)$ and the luminosity limit $\left(L_{\min }\right)$.

\begin{tabular}{llllllll} 
GRB & $F$ & $T$ & $N$ & $t$ & $D_{L}$ & $z$ & $L_{\text {min }}$ \\
\hline & $(\mathrm{MHz})$ & (hours) & & (hours) & $(\mathrm{Mpc})$ & & $(\mathrm{erg} / \mathrm{s} / \mathrm{Hz})$ \\
\hline 030329 & 1380 & 4.5 & 7 & 0.65 & 812 & 0.169 & $6.9 \times 10^{31}$ \\
& 4500 & 1.7 & 4 & 0.42 & & & \\
051221 & 1380 & 14.7 & 15 & 0.98 & 2868 & 0.547 & $7.2 \times 10^{32}$ \\
& 4500 & 2.5 & 5 & 0.51 & & & \\
060218 & 1380 & 8.7 & 16 & 0.55 & 145 & 0.33 & $1.4 \times 10^{30}$ \\
& 4500 & 6.9 & 13 & 0.53 & & & \\
$120422 \mathrm{~A}$ & 1380 & 3.5 & 4 & 0.88 & 1434 & 0.280 & $1.2 \times 10^{32}$ \\
& 4500 & 1.2 & 2 & 0.60 & & & \\
$130427 \mathrm{~A}$ & 1380 & 2.7 & 4 & 0.67 & 1795 & 0.340 & $2.1 \times 10^{32}$ \\
& 4500 & 1.0 & 2 & 0.51 & & & \\
$130702 \mathrm{~A}$ & 1380 & 13.7 & 21 & 0.65 & 687 & 0.145 & $2.3 \times 10^{31}$ \\
& 4500 & 6.9 & 15 & 0.46 & & & \\
$130215 \mathrm{~A}$ & 1380 & 12.1 & 19 & 0.64 & 3508 & 0.597 & $1.1 \times 10^{33}$ \\
& 4500 & 7.5 & 12 & 0.63 & & & \\
$130603 \mathrm{~B}$ & 1380 & 2.3 & 3 & 0.76 & 1894 & 0.356 & $2.4 \times 10^{32}$ \\
& 4500 & 1.0 & 2 & 0.50 & & & \\
$140903 \mathrm{~A}$ & 1380 & 4.1 & 6 & 0.68 & 1863 & 0.351 & $2.3 \times 10^{32}$ \\
& 4500 & 3.1 & 6 & 0.51 & & & \\
051221 & 1380 & 14.7 & 15 & 0.98 & 2868 & 0.547 & $7.2 \times 10^{32}$ \\
& 4500 & 2.5 & 5 & 0.51 & & & \\
$100816 \mathrm{~A}$ & 1380 & 5.4 & 8 & 0.67 & 4529 & 0.803 & $2.4 \times 10^{33}$ \\
& 4500 & 1.3 & 3 & 0.44 & & & \\
$130831 \mathrm{~A}$ & 1380 & 10.3 & 13 & 0.79 & 2459 & 0.479 & $4.8 \times 10^{32}$ \\
& 4500 & 4.9 & 8 & 0.62 & & & \\
\hline
\end{tabular}


distinguish between RFI and real transients are also in place. If FRBs are indeed related to explosive events, better understanding of the emission process and the environment of the explosion will help determine factors such as time for radiation to escape and thereby a observing cadence for future targeted searches.

\subsection{Going Forward}

An important question regarding the FRB population that we have begun to address in this work is what are the statistics of source numbers versus source flux density - the $\log N-\log S$ curve. To answer this question, it is particularly important to sample the extreme ends of the flux density axis: the brightest FRB discovered using small telescopes such as the Green Bank 20-m telescope in long duration and large sky-coverage surveys, as well as the weakest FRBs sampled through high-sensitivity observations with large telescopes like Arecibo, necessarily sacrificing survey time and sky coverage. Despite its small instantaneous field of view, the Arecibo telescope is still playing a crucial role in continuing the FRB searches due to its high sensitivity and diverse receivers. The ALFA receiver is going to be decommissioned in 2021 and replaced with "AO40" phased-array feed receiver with a much larger field of view. The planned 40-beam system will have a similar sensitivity to ALFA and over five times the field of view. Real-time processing pipelines are already realized at other telescopes, and Arecibo's smaller beam would make follow-up at other wavelengths easier.

The current 100 known FRBs vary significantly in DM, RM, pulse width and 
shape, and flux density. Even though the wide-field radio telescopes have more than doubled with the number of detected FRBs over only the last two years, a large number of questions remain unanswered such as their emission mechanism and population properties. Moreover, the repeatability and multi-wavelength emission of FRBs will still remain a mystery. Meanwhile, we are entering a new era in FRB astronomy, when innovative techniques are emerging with the help of a new generation of telescopes. Even though radio telescopes with large fields-of-view like CHIME and ASKAP are dominating FRB searches, single-dish telescopes such as the GBT and Arecibo will continue to play a crucial role in follow-up searches at targeted locations. For instance, the Five-hundred-meter Aperture Spherical radio Telescope (FAST) in China, is expected to go even deeper than Arecibo in terms of redshift coverage. At the time of writing, rumors abound with the imminent release of a catalog of over 700 FRBs from the first year of CHIME operations. The future of FRB science is, therefore, very bright. We anticipate that the combination of upcoming surveys with these powerful new instruments and proposed multi-wavelength searches at existing telescopes will result in substantial progress over the next decade. 


\section{Bibliography}

Abramowicz, M. A., Bejger, M., \& Wielgus, M. 2018, ApJ, 868, 17

Agarwal, D., Aggarwal, K., Burke-Spolaor, S., Lorimer, D. R., \& Garver-Daniels, N. 2019, arXiv e-prints, arXiv:1902.06343

Agarwal, D., Lorimer, D. R., Surnis, M. P., et al. 2020, arXiv e-prints, arXiv:2003.14272

Akahori, T., \& Ryu, D. 2010, The Astrophysical Journal, 723, 476481. http: //dx.doi.org/10.1088/0004-637X/723/1/476

-. 2011, The Astrophysical Journal, 738, 134. http://dx.doi.org/10.1088/ $0004-637 \mathrm{X} / 738 / 2 / 134$

Amiri, M., Andersen, B., Bandura, K., et al. 2020, arXiv preprint arXiv:2001.10275

Auld, R., Minchin, R. F., Davies, J. I., et al. 2006, MNRAS, 371, 1617

Bagchi, M., Nieves, A. C., \& McLaughlin, M. 2012, MNRAS, 425, 2501

Bannister, K. W., \& Madsen, G. J. 2014, MNRAS, 440, 353

Bannister, K. W., Murphy, T., Gaensler, B. M., \& Reynolds, J. E. 2012, The Astrophysical Journal, 757, 38

Bannister, K. W., Deller, A. T., Phillips, C., et al. 2019, arXiv e-prints, arXiv:1906.11476

Barrau, A., Rovelli, C., \& Vidotto, F. 2014, Phys. Rev. D, 90, 127503 
Barsdell, B. R. 2012, PhD thesis, Swinburne University of Technology

Barthelmy, S. 2015, in APS Meeting Abstracts, Vol. 2015, APS April Meeting Abstracts, L1.012

Bennett, C. L., Larson, D., Weiland, J. L., \& Hinshaw, G. 2014, ApJ, 794, 135

Bhandari, S., Keane, E. F., Barr, E. D., et al. 2018, MNRAS, 475, 1427

Bochenek, C. D., McKenna, D. L., Belov, K. V., et al. 2020, PASP, 132, 034202

Burke-Spolaor, S., Bailes, M., Ekers, R., Macquart, J.-P., \& Crawford, III, F. 2011, ApJ, 727, 18

Burke-Spolaor, S., \& Bannister, K. W. 2014, ApJ, 792, 19

Burke-Spolaor, S., Trott, C. M., Brisken, W. F., et al. 2016, ApJ, 826, 223

Caleb, M., Flynn, C., Bailes, M., et al. 2016, MNRAS, 458, 708

Caleb, M., Stappers, B. W., Rajwade, K., \& Flynn, C. 2019, MNRAS, 484, 5500

Caleb, M., Flynn, C., Bailes, M., et al. 2016, Monthly Notices of the Royal Astronomical Society, 458, 718

Caleb, M., Flynn, C., Bailes, M., et al. 2017, MNRAS, 468, 3746

Caleb, M., Flynn, C., Bailes, M., et al. 2017, Monthly Notices of the Royal Astronomical Society, 468, 3746

Champion, D. J., Petroff, E., Kramer, M., et al. 2016, MNRAS, 460, L30 
Chatterjee, S., Law, C. J., Wharton, R. S., et al. 2017, Nature, 541, 58

Chawla, P., Kaspi, V. M., Josephy, A., et al. 2017, ApJ, 844, 140

Chen, G., \& Ratra, B. 2011, PASP, 123, 1127

Chennamangalam, J., MacMahon, D., Cobb, J., et al. 2017, ApJS, 228, 21

CHIME/FRB Collaboration, Amiri, M., Bandura, K., et al. 2019a, Nature, 566, 235

—. 2019b, Nature, 566, 230

Coenen, T., van Leeuwen, J., Hessels, J. W. T., et al. 2014, Astronomy \& Astrophysics, 570, A60. https://doi.org/10.1051/0004-6361/201424495

Coenen, T., van Leeuwen, J., Hessels, J. W. T., et al. 2014, A\&A, 570, A60

Colgate, S. 1975, The Astrophysical Journal, 198, 439

Colgate, S. A., \& Noerdlinger, P. D. 1971, The Astrophysical Journal, 165, 509

Condon, J. J., \& Ransom, S. M. 2016, Essential Radio Astronomy (Princeton)

Connor, L., Sievers, J., \& Pen, U.-L. 2016, MNRAS, 458, L19

Connor, L., \& van Leeuwen, J. 2018, AJ, 156, 256

Cordes, J., \& McLaughlin, M. A. 2003, The Astrophysical Journal, 596, 1142

Cordes, J. M., \& Chatterjee, S. 2019, ARA\&A, 57, 417

Cordes, J. M., \& Chatterjee, S. 2019, Annual Review of Astronomy and Astrophysics, 57,417 
Cordes, J. M., \& Lazio, T. J. W. 2002, arXiv e-prints, astro

Cordes, J. M., \& Lazio, T. J. W. 2002, arXiv preprint astro-ph/0207156

Cordes, J. M., \& Wasserman, I. 2016, MNRAS, 457, 232

Cordes, J. M., Freire, P. C. C., Lorimer, D. R., et al. 2006, ApJ, 637, 446

Crawford, F., Rane, A., Tran, L., et al. 2016, MNRAS, 460, 3370

Dai, Z. G., Wang, J. S., Wu, X. F., \& Huang, Y. F. 2016, ApJ, 829, 27

Dainotti, M. G., Hernandez, X., Postnikov, S., et al. 2017, ApJ, 848, 88

DeLaunay, J., Fox, D. B., Murase, K., et al. 2016, The Astrophysical Journal Letters, 832, L1

Deneva, J. S., Stovall, K., McLaughlin, M. A., et al. 2016, ApJ, 821, 10

Devine, T. R., Goseva-Popstojanova, K., \& McLaughlin, M. 2016, Monthly Notices of the Royal Astronomical Society, 459, 1519

Dokuchaev, V. I., \& Eroshenko, Y. N. 2017, arXiv e-prints, arXiv:1701.02492

Eatough, R. P., Keane, E. F., \& Lyne, A. G. 2009, MNRAS, 395, 410

Egorov, A. E., \& Postnov, K. A. 2009, Astronomy Letters, 35, 241

Falcke, H., \& Rezzolla, L. 2014, Astronomy \& Astrophysics, 562, A137

Falcke, H., \& Rezzolla, L. 2014, A\&A, 562, A137

Farah, W., Flynn, C., Bailes, M., et al. 2018, MNRAS, 478, 1209 
Farah, W., Flynn, C., Bailes, M., et al. 2018, Monthly Notices of the Royal Astronomical Society, 478, 1209

—. 2019, arXiv preprint arXiv:1905.02293

Foster, G., Karastergiou, A., Golpayegani, G., et al. 2018, MNRAS, 474, 3847

Frail, D. A., Kulkarni, S. R., Sari, R., et al. 2001, ApJL, 562, L55

Fuller, J., \& Ott, C. D. 2015, MNRAS, 450, L71

Geng, J. J., \& Huang, Y. F. 2015, ApJ, 809, 24

Golpayegani, G., Lorimer, D. R., Ellingson, S. W., et al. 2019, MNRAS, 489, 4001

Gu, W.-M., Dong, Y.-Z., Liu, T., Ma, R., \& Wang, J. 2016, ApJL, 823, L28

Gupta, P. D., \& Saini, N. 2018, Journal of Astrophysics and Astronomy, 39, 14

Gutenberg, B., \& Richter, C. F. 1956, Bulletin of the seismological society of America, 46, 105

Hewish, A., Bell, S. J., Pilkington, J. D., Scott, P. F., \& Collins, R. A. 1968, Nature, 217,709

Hogg, D. W. 1999, arXiv e-prints, astro

Hosmer, L., Langston, G., Heatherly, S., et al. 2013, in American Astronomical Society Meeting Abstracts, Vol. 221, American Astronomical Society Meeting Abstracts \#221, 345.24 
Huchra, J. P., \& Geller, M. J. 1982, ApJ, 257, 423

Ioka, K. 2003, The Astrophysical Journal Letters, 598, L79

Istomin, Y. N. 2018, MNRAS, 478, 4348

James, C. W., Ekers, R. D., Macquart, J.-P., Bannister, K. W., \& Shannon, R. M. 2019, MNRAS, 483, 1342

Karako-Argaman, C., Kaspi, V. M., Lynch, R. S., et al. 2015, ApJ, 809, 67

Karastergiou, A., Chennamangalam, J., Armour, W., et al. 2015, MNRAS, 452, 1254

Kashiyama, K., Ioka, K., \& Mszros, P. 2013, The Astrophysical Journal Letters, 776, L39. http://stacks.iop.org/2041-8205/776/i=2/a=L39

Kaspi, V. M., \& Beloborodov, A. M. 2017, Annual Review of Astronomy and Astrophysics, 55, 261

Katz, J. I. 2016, Modern Physics Letters A, 31, 1630013

Keane, E., Stappers, B., Kramer, M., \& Lyne, A. 2012, Monthly Notices of the Royal Astronomical Society: Letters, 425, L71

Keane, E. F., Kramer, M., Lyne, A., Stappers, B., \& McLaughlin, M. 2011, Monthly Notices of the Royal Astronomical Society, 415, 3065

Keane, E. F., Barr, E. D., Jameson, A., et al. 2018, MNRAS, 473, 116

Kibble, T. W. 1980, Physics Reports, 67, 183 
Kocz, J., Bailes, M., Barnes, D., Burke-Spolaor, S., \& Levin, L. 2012, MNRAS, 420, 271

Kulkarni, S. R., \& Narayan, R. 1988, ApJ, 335, 755

Kumar, P., Shannon, R. M., Osłowski, S., et al. 2019, ApJL, 887, L30

Law, C., Margalit, B., Palliyaguru, N. T., et al. 2019, BAAS, 51, 319

Law, C. J., Bower, G. C., Burke-Spolaor, S., et al. 2015, ApJ, 807, 16

Law, C. J., Bower, G. C., Burke-Spolaor, S., et al. 2018, The Astrophysical Journal Supplement Series, 236, 8. http://stacks.iop.org/0067-0049/236/i=1/a=8

Lawrence, E., Vander Wiel, S., Law, C., Burke Spolaor, S., \& Bower, G. C. 2017, AJ, 154, 117

Li, L.-B., Huang, Y.-F., Zhang, Z.-B., Li, D., \& Li, B. 2017, Research in Astronomy and Astrophysics, 17, 6

Lingam, M., \& Loeb, A. 2017, ApJL, 837, L23

Liu, T., Romero, G. E., Liu, M.-L., \& Li, A. 2016, ApJ, 826, 82

Lorimer, D. R., Bailes, M., McLaughlin, M. A., Narkevic, D. J., \& Crawford, F. 2007, Science, 318, 777

Lorimer, D. R., Karastergiou, A., McLaughlin, M. A., \& Johnston, S. 2013, MNRAS, 436, L5

Lorimer, D. R., \& Kramer, M. 2004, Handbook of Pulsar Astronomy (CUP) 
Luan, J., \& Goldreich, P. 2014, ApJL, 785, L26

Luo, R., Lee, K., Lorimer, D. R., \& Zhang, B. 2018, MNRAS, 481, 2320

Lyubarsky, Y. 2014, MNRAS, 442, L9

Lyutikov, M., Burzawa, L., \& Popov, S. B. 2016, MNRAS, 462, 941

Maan, Y., \& van Leeuwen, J. 2017, ArXiv e-prints, arXiv:1709.06104

Macquart, J.-P., \& Ekers, R. D. 2018, MNRAS, 474, 1900

Macquart, J.-P., \& Koay, J. Y. 2013, ApJ, 776, 125

Macquart, J.-P., Bailes, M., Bhat, N. D. R., et al. 2010, PASA, 27, 272

Madau, P., \& Dickinson, M. 2014, ARA\&A, 52, 415

Manchester, R. N., Hobbs, G. B., Teoh, A., \& Hobbs, M. 2005, AJ, 129, 1993

Manchester, R. N., Lyne, A. G., Camilo, F., et al. 2001, MNRAS, 328, 17

Marcote, B., Nimmo, K., Hessels, J. W. T., et al. 2020, arXiv e-prints, arXiv:2001.02222

Masui, K., Lin, H.-H., Sievers, J., et al. 2015, Nature, 528, 523

Metzger, B. D., Berger, E., \& Margalit, B. 2017, ApJ, 841, 14

Metzger, B. D., Margalit, B., \& Sironi, L. 2019, MNRAS, 485, 4091

Michilli, D., Seymour, A., Hessels, J. W. T., et al. 2018, Nature, 553, 182 
Mingarelli, C. M. F., Levin, J., \& Lazio, T. J. W. 2015, ApJL, 814, L20

Most, E. R., Nathanail, A., \& Rezzolla, L. 2018, ApJ, 864, 117

Mottez, F., \& Zarka, P. 2014, A\&A, 569, A86

Nicholl, M., Williams, P. K. G., Berger, E., et al. 2017, ApJ, 843, 84

Nielsen, H. B., \& Olesen, P. 1973, Nuclear Physics B, 61, 45

Nita, G. M., \& Gary, D. E. 2010, MNRAS, 406, L60

Noutsos, A. 2012, Space science reviews, 166, 307

Oppermann, N., Connor, L. D., \& Pen, U.-L. 2016, MNRAS, 461, 984

Palaniswamy, D., Wayth, R. B., Trott, C. M., et al. 2014, The Astrophysical Journal, 790,63

Pang, D., Goseva-Popstojanova, K., Devine, T., \& McLaughlin, M. 2018, Monthly Notices of the Royal Astronomical Society, 480, 3302

Patel, C., Agarwal, D., Bhardwaj, M., et al. 2018, The Astrophysical Journal, 869, 181

Peek, J., Heiles, C., Douglas, K. A., et al. 2011, The Astrophysical Journal Supplement Series, 194, 20

Petroff, E., Hessels, J. W. T., \& Lorimer, D. R. 2019, A\&A Rev., 27, 4

Petroff, E., van Straten, W., Johnston, S., et al. 2014, ApJL, 789, L26 
Petroff, E., Keane, E., Barr, E., et al. 2015, Monthly Notices of the Royal Astronomical Society, 451, 3933

Petroff, E., Barr, E. D., Jameson, A., et al. 2016, PASA, 33, e045

Phinney, S., \& Taylor, J. H. 1979, Nature, 277, 117

Platts, E., Weltman, A., Walters, A., et al. 2018, arXiv e-prints, arXiv:1810.05836

Popov, S. B., \& Postnov, K. A. 2010, in Evolution of Cosmic Objects through their Physical Activity, ed. H. A. Harutyunian, A. M. Mickaelian, \& Y. Terzian, $129-132$

Rajwade, K., Chennamangalam, J., Lorimer, D., et al. 2016, arXiv preprint arXiv:1602.04389

Rajwade, K. M., \& Lorimer, D. R. 2017, MNRAS, 465, 2286

Rane, A. 2017, PhD thesis, West Virginia University

Rane, A., Lorimer, D. R., Bates, S. D., et al. 2016, MNRAS, 455, 2207

Ravi, V. 2019, Nature Astronomy, 405

Ravi, V., Shannon, R., Bailes, M., et al. 2016, Science, 354, 1249

Ravi, V., Catha, M., D’Addario, L., et al. 2019, arXiv e-prints, arXiv:1907.01542

Rees, M. J. 1977, Nature, 266, 333

Ryu, D., Kang, H., Cho, J., \& Das, S. 2008, Science, 320, 909 
Saint-Hilaire, P., Benz, A. O., \& Monstein, C. 2014, ApJ, 795, 19

Schechter, P. 1976, ApJ, 203, 297

Scheuer, P. 1968, in Pulsating Stars (Springer), 86-88

Scholz, P., Spitler, L., Hessels, J., et al. 2016, The Astrophysical Journal, 833, 177

Shannon, R. M., Macquart, J.-P., Bannister, K. W., et al. 2018, Nature, 562, 386

Siemion, A. P. V., Bower, G. C., Foster, G., et al. 2012, ApJ, 744, 109

Smith, A. B., Caton, D. B., \& Hawkins, R. L. 2016, PASP, 128, 055002

Spitler, L., Cordes, J., Hessels, J., et al. 2014, The Astrophysical Journal, 790, 101

Spitler, L., Scholz, P., Hessels, J., et al. 2016, Nature, 531, 202

Spitler, L. G., Cordes, J. M., Hessels, J. W. T., et al. 2014, ApJ, 790, 101

Spitler, L. G., Scholz, P., Hessels, J. W. T., et al. 2016, Nature, 531, 202

Stappers, B. 2016, in Proceedings of MeerKAT Science: On the Pathway to the SKA. 25-27 May, 2016 Stellenbosch, South Africa (MeerKAT2016). Online at ¡A href="href="¿href="https://pos.sissa.it/cgibin/reader/conf.cgi?confid=277i/Ai, id.10, 10

Stovall, K., Lynch, R. S., Ransom, S. M., et al. 2014, ApJ, 791, 67

Surnis, M. P., Agarwal, D., Lorimer, D. R., et al. 2019, arXiv e-prints, arXiv:1903.05573 
Tauris, T. M., \& Manchester, R. N. 1998, MNRAS, 298, 625

Taylor, R., \& Salter, C. 2010, arXiv preprint arXiv:1008.4944

Tendulkar, S. P., Bassa, C. G., Cordes, J. M., et al. 2017, ApJL, 834, L7

The CHIME/FRB Collaboration, :, Amiri, M., et al. 2018, ArXiv e-prints, arXiv:1803.11235

The CHIME/FRB Collaboration, :, Andersen, B. C., et al. 2019, arXiv e-prints, arXiv:1908.03507

Thornton, D., Stappers, B., Bailes, M., et al. 2013, Science, 341, 53

Tingay, S. J., Trott, C. M., Wayth, R. B., et al. 2015, AJ, 150, 199

Totani, T. 2013, PASJ, 65, L12

Vachaspati, T. 2008, Phys. Rev. Lett., 101, 141301

Van Waerbeke, L., \& Zhitnitsky, A. 2019, Phys. Rev. D, 99, 043535

Vazza, F., Brüggen, M., Hinz, P. M., et al. 2018, MNRAS, 480, 3907

Vedantham, H. K., Ravi, V., Hallinan, G., \& Shannon, R. M. 2016, ApJ, 830, 75

Vieyro, F. L., Romero, G. E., Bosch-Ramon, V., Marcote, B., \& del Valle, M. V. 2017, A\&A, 602, A64

Wang, J.-S., Yang, Y.-P., Wu, X.-F., Dai, Z.-G., \& Wang, F.-Y. 2016, ApJL, 822, L7 
Wang, W., Luo, R., Yue, H., et al. 2018, ApJ, 852, 140

Wayth, R. B., Brisken, W. F., Deller, A. T., et al. 2011, ApJ, 735, 97

Williamson, I. P. 1972, MNRAS, 157, 55

Yamasaki, S., Totani, T., \& Kiuchi, K. 2018, PASJ, 70, 39

Yang, Y.-P., \& Zhang, B. 2016, The Astrophysical Journal Letters, 830, L31

Ye, J., Wang, K., \& Cai, Y.-F. 2017, European Physical Journal C, 77, 720

Zhang, B. 2013, The Astrophysical Journal Letters, 780, L21

Zhang, B. 2016, ApJL, 827, L31

—. 2017, ApJL, 836, L32

Zhang, S., Hobbs, G., Dai, S., et al. 2019, Monthly Notices of the Royal Astronomical Society: Letters, 484, L147

Zheng, Z., Ofek, E., Kulkarni, S., Neill, J., \& Juric, M. 2014, The Astrophysical Journal, 797, 71

Zhitnitsky, A. R. 2003, JCAP, 2003, 010

Zhu, W., Feng, L.-L., \& Zhang, F. 2018, ApJ, 865, 147 